\title{
The Sphere Packing Bound For Memoryless Channels
}

Barış Nakiboğlu

bnakib@metu.edu.tr

\begin{abstract}
Sphere packing bounds (SPBs) — with prefactors that are polynomial in the block length — are derived for codes on two families of memoryless channels using Augustin's method: (possibly non-stationary) memoryless channels with (possibly multiple) additive cost constraints and stationary memoryless channels with convex constraints on the composition (i.e. empirical distribution, type) of the input codewords. A variant of Gallager's bound is derived in order to show that these sphere packing bounds are tight in terms of the exponential decay rate of the error probability with the block length under mild hypotheses.
\end{abstract}

\section{CONTENTS}

1 Introduction 1 .1 2

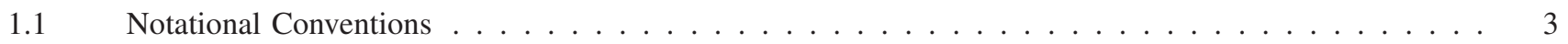

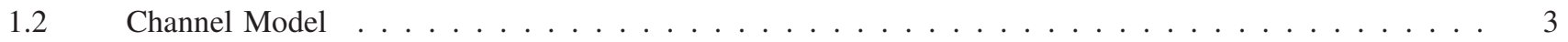

1.3 Codes With List Decoding . . . . . . . . . . . . . . . . . . . 4

2 Preliminaries 4

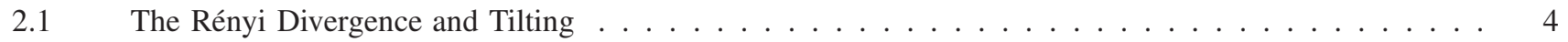

2.2 Augustin's Information Measures . . . . . . . . . . . . . . . . . . . . 5

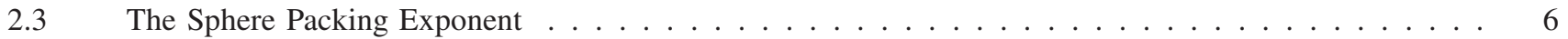

2.4 Augustin's Variant of Gallager's Bound . . . . . . . . . . . . . . . . . . . . . 8

3 SPBs For Memoryless Channels 11

$3.1 \quad$ An Impossibility Result For Hypothesis Testing . . . . . . . . . . . . . . . . . . . . . . 11

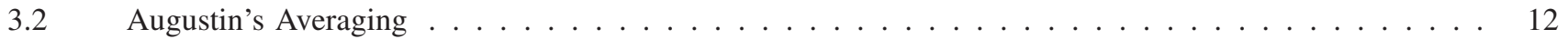

3.3 Non-asymptotic SPBs for the Composition Constrained Codes . . . . . . . . . . . . . . . . . . . . 12

$3.4 \quad$ Non-asymptotic SPBs for the Cost Constrained Codes . . . . . . . . . . . . . . . . . . . . . . . . 15

4 Examples 17

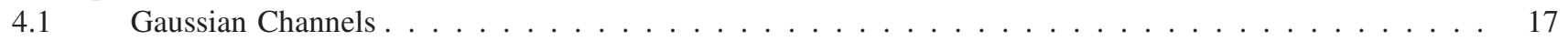

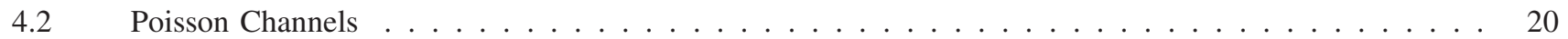

5 Discussion 21

Appendix 22

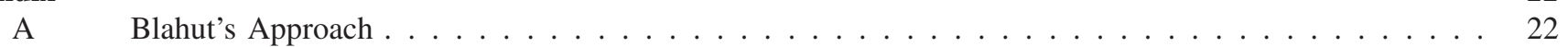

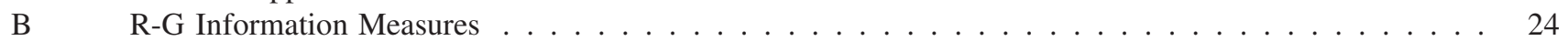

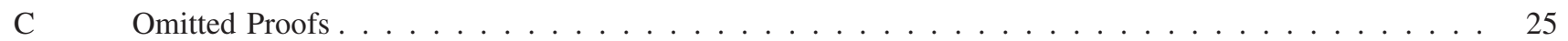

References 28 


\section{INTRODUCTION}

Most proofs of the sphere packing bound (SPB) have been either for the stationary channels with finite input sets [2]-[14] or for the stationarity channels with a specific noise structure, e.g. Poisson, Gaussian, [15]-[23]. Proofs of the SPB based on Augustin's method are exceptions to this observation: [24]-[26] do not assume either the finiteness of the input set or a specific noise structure, nor do they assume the stationarity of the channel. However, [24], [25, §31], [26] establish the SPB for the product channels, rather than the memoryless channels; hence proofs of the SPB for the composition constrained codes ${ }^{1}$ on the stationary channels [9]-[20] — which include the important special case of the cost constrained ones [15]-[20]— are not subsumed by [24], [25, §31], or [26]. In [25, §36], Augustin proved the SPB for the cost constrained (possibly non-stationary) memoryless channels assuming a bounded cost function. The framework of [25, Thm. 36.6] subsumes all previously considered models [2]-[23], except the Gaussian ones [17]-[20].

Theorem 2, presented in $\S 3$, establishes the SPB for a framework that subsumes all of the models considered in [2]-[26] by employing [27], which analyzes Augustin's information measures. Our use of [27] and Augustin's information measures is similar to the use of [28] and Rényi's information measures in [26]. For the product channels, [26, Thm. 2] improved the previous results by Augustin in [24], [25, §31] by establishing the SPB with a prefactor that is polynomial in the block length $n$ for the hypothesis that the order $1 / 2$ Rényi capacity of the component channels are $O(\ln n)$. For the cost constrained memoryless channels, Theorem 2 enhances the prefactor of [25, Thm. 36.6] in an analogous way, from $e^{-O(\sqrt{n})}$ to $e^{-O(\ln n)}$. The prefactor of Theorem 2, however, is inferior to the prefactors reported in [3]-[6] for various symmetric channels, in [13] for the constant composition codes on discrete stationary product channels, in [17] for the stationary Gaussian channel, and in [21]-[23] for certain non-coherent fading channels. Determination of the optimal prefactor, in the spirit of [3]-[5], remains an open problem for the general case. ${ }^{2}$ Similar to [25, Thm. 36.6], Theorem 2 holds for non-stationary channels, as well. Unlike [25, Thm. 36.6], Theorem 2 does not assume the cost functions to be bounded.

The stationarity is assumed in most of the previous derivations of the SPB, [2]-[23]. Given a stationary product channel, one can obtain a stationary memoryless channel by imposing composition - i.e. type, empirical distribution- constraints on the input codewords. The cost constraints can be interpreted as a particular convex case of this more general composition constraints. This interpretation, considered together with the composition based expurgations, is one of the main motivating factors behind the study of constant composition codes. The composition based expurgations, however, are useful only when the input set of the channel is finite. Nevertheless, if the constraint set for the composition of the codewords is convex, then one can derive a SPB with a polynomial prefactor using Augustin's information measures, see Theorem 1 in $\S 3$. The derivation of Theorem 1 relies on the Augustin center of the constraint set rather than the Augustin mean of the most populous composition of the code. Note that the most populous composition of the code might not even have more than one codeword when the input set is infinite. The framework of Theorem 1 is general enough to subsume the frameworks of all previous proofs of the SPB for the memoryless channels that we are aware of, except the frameworks of the proofs based on Augustin's method [24]-[26]. Theorems 1 and 2 are asymptotic SPBs; but they are proved using non-asymptotic SPBs presented in Lemmas 9 and 10 .

The SPB implies that exponential decay rate of the optimal error probability with the block length -i.e. the reliability function, the error exponent - is bounded from above by the sphere packing exponent (SPE). For the memoryless channels in consideration, Augustin's variant of Gallager's bound implies that the SPE bounds the reliability function from below, as well, provided that the list decoding is allowed. Augustin's variant of Gallager's bound is presented in \$2.4. One can use standard results such as [30], [31] with minor modifications in order to establish the SPE as a lower bound to the reliability function for the list decoding, as well. Thus Augustin's variant is of interest to us not because of what it implies about the reliability function but because of how it implies it. What is unique about Augustin's variant is that it establishes an achievability result in terms of the Augustin information rather than the Rényi information used in the standard form of the Gallager's bound [30]. Augustin's variant relies on the fixed point property of the Augustin mean described in (7) to do that. It is worth mentioning that [31] implicitly employs the same fixed point property but in a different way.

Before starting our discussion in earnest, let us point out a subtlety about the derivations of the SPB that is usually overlooked. [32] claimed to prove the SPB for arbitrary stationary product channels, without using any constant composition arguments. ${ }^{3}$ The derivation of [32, Thm. 19], however, establishes an upper bound on the reliability function that is strictly greater than the SPE in many channels. This has been demonstrated numerically in [13, p. 1594 and Appendix A]. An analytic confirmation this observation is presented in Appendix A. The problematic step in [32] is the application of Lagrange multiplier techniques, see [13, footnote 8]. The proof of [32, Thm. 19] invokes [32, Thm. 16] that is valid for the Lagrange multiplier $s$ associated with the input distribution $p$ satisfying $E_{s p}(R, W)=E_{s p}(R, W, p)$. For an arbitrary input distribution $p$, however, the associated Lagrange multiplier may or may not be equal to the one for the optimal input distribution $p$. This is the reason why the

\footnotetext{
${ }^{1}$ According to [12, p. 183], the SPB for the constant composition codes appears in [9] with an incomplete proof. The first complete proof of the SPB for the constant composition codes is provided in [10].

${ }^{2}$ Elsewhere in [29], we have derived refined SPBs (which are optimal in terms of the prefactor for non-singular cases) for all of the cases considered in [3]-[6], [13], [17], [21]-[23] using Augustin information measures via [27].

3 [32, p. 413] reads "An important feature of the lower bound, which will be derived, is that no assumption of constant-composition codewords is made, not even as an intermediate step."
} 
upper bound to the reliability function established in [32, Thm. 19] is not equal to the SPE in general, contrary to the claim repeated in [33, Lemma 1] and [34, Thm. 10.1.4]. In a nutshell, the proof of [32, Thm. 19] tacitly asserts a minimax equality that does not hold in general. For stationary memoryless channels with finite input alphabets, one can avoid this issue using the constant composition arguments. However, in that case, the proof presented in [32] becomes a mere reproduction of the one in [10]. More recently, [35] proposed a derivation of the SPB for stationary channels with a single cost constraint using the approach presented in [32]. Similar to [32], however, the proof in [35] asserts a minimax equality that does not hold in general. In particular, it is claimed that $Q^{n}$ does not depend on $\mathbf{x}_{m}$ in $[35,(26)]$. To assert that, one has to include an additional supremum over $\mathbf{x}_{m}$ as the innermost optimization in both [35, (25) and (26)]. With the additional supremum, the explanation provided on [35, p. 931] is no longer valid. Considering Appendix A, we do not believe that the proof in [35] can be salvaged without introducing major new ideas, such as composition based expurgations similar to [10] or codeword cost based expurgations similar to [18]. In short, neither [32] nor [35] successfully proved the SPB for stationary memoryless channels even for the finite input set case.

In the rest of this section, we introduce our notation and channel model and define the channel codes with list decoding. In $\S 2$, we first present a brief review of the Rényi divergence, Augustin information measures, and the SPE; then, we derive Augustin's variant of Gallager's bound. In $\$ 3$, we first state our main asymptotic results -i.e., SPBs given in Theorem 1 and Theorem 2- and then derive the non-asymptotic SPBs implying them. In §4, we derive the SPE for particular Gaussian and Poisson channels and confirm the equivalence of the definition invoked in $\$ 2$ to the ones derived for these channels previously. In $§ 5$, we discuss why Augustin's method works briefly and compare our results with Augustin's in [25] and discuss applications of Augustin's method and use of Augustin's information measures in related problems.

\subsection{Notational Conventions}

For any two vectors $\mu$ and $q$ in $\mathbb{R}^{\ell}$ their inner product, denoted by $\mu \cdot q$, is $\sum_{\imath=1}^{\ell} \mu^{\imath} q^{\imath}$. For any $\ell \in \mathbb{Z}_{+}, \ell$ dimensional vector whose all entries are one is denoted by $\mathbb{1}$, the dimension $\ell$ will be clear from the context. We denote the closure, interior, and convex hull of a set $\mathcal{S}$ by $c 1 \mathcal{S}$, int $\mathcal{S}$, and $\mathrm{ch} \mathcal{S}$, respectively; the relevant topology or vector space structure will be evident from the context.

For any set $y$, we denote the set of all probability mass functions that are non-zero only on finitely many members of $y$ by $\mathcal{P}(y)$. For any $p \in \mathcal{P}(y)$, we call the set of all $y$ 's in $y$ for which $p(y)>0$ the support of $p$ and denote it by supp $(p)$. For any measurable space $(\mathcal{Y}, \mathcal{Y})$, we denote the set of all probability measures on it by $\mathcal{P}(\mathcal{Y})$ and set of all finite measures by $\mathcal{M}^{+}(\mathcal{Y})$. We denote the integral of a measurable function $f$ with respect to the measure $\mu$ by $\int f \mu(\mathrm{d} y)$ or $\int f(y) \mu(\mathrm{d} y)$. If the integral is on the real line and if it is with respect to the Lebesgue measure, we denote it by $\int f \mathrm{~d} y$ or $\int f(y) \mathrm{d} y$, as well. If $\mu$ is a probability measure, then we also call the integral of $f$ with respect $\mu$ the expectation of $f$ or the expected value of $f$ and denote it by $\mathbf{E}_{\mu}[f]$ or $\mathbf{E}_{\mu}[f(\mathrm{Y})]$.

Our notation will be overloaded for certain symbols; however, the relations represented by these symbols will be clear from the context. We denote the Cartesian product of sets [36, p. 38] by $\times$. We use $|\cdot|$ to denote the absolute value of real numbers and the size of sets. The sign $\leq$ stands for the usual less than or equal to relation for real numbers and the corresponding point-wise inequity for functions and vectors. For two measures $\mu$ and $q$ on the measurable space $(\mathcal{y}, \mathcal{Y}), \mu \leq q$ iff $\mu(\mathcal{E}) \leq q(\mathcal{E})$ for all $\mathcal{E} \in \mathcal{Y}$. We denote the product of topologies [36, p. 38], $\sigma$-algebras [36, p. 118], and measures [36, Thm. 4.4.4] by $\otimes$. We use the shorthand $X_{1}^{n}$ for the Cartesian product of sets $X_{1}, \ldots, X_{n}$ and $\mathcal{Y}_{1}^{n}$ for the product of the $\sigma$-algebras $\mathcal{Y}_{1}, \ldots, \mathcal{Y}_{n}$.

\subsection{Channel Model}

A channel $W$ is a function from the input set $\mathcal{X}$ to the set of all probability measures on the output space ( $\mathcal{Y}, \mathcal{Y})$ :

$$
W: X \rightarrow \mathcal{P}(\mathcal{Y})
$$

$y$ is called the output set, and $\mathcal{Y}$ is called the $\sigma$-algebra of the output events. We denote the set of all channels from the input set $\mathcal{X}$ to the output space $(\mathcal{Y}, \mathcal{Y})$ by $\mathcal{P}(\mathcal{Y} \mid X)$. For any $p \in \mathcal{P}(\mathcal{X})$ and $W \in \mathcal{P}(\mathcal{Y} \mid X), p \circledast W$ is the probability measure whose marginal on $\mathcal{X}$ is $p$ and whose conditional distribution given $x$ is $W(x)$. The structure described in (1) is not sufficient on its own to ensure the existence of a unique $p \circledast W$ with the desired properties for all $p \in \mathcal{P}(\mathcal{X})$, in general. The existence of a unique $p \circledast W$ is guaranteed for all $p \in \mathcal{P}(\mathcal{X})$, if $W$ is a transition probability from $(\mathcal{X}, \mathcal{X})$ to $(\mathcal{Y}, \mathcal{Y})$, i.e. a member of $\mathcal{P}(\mathcal{Y} \mid \mathcal{X})$ rather than $\mathcal{P}(\mathcal{Y} \mid \mathcal{X})$.

A channel $W$ is called a discrete channel if both $\mathcal{X}$ and $\mathcal{Y}$ are finite sets. For any $n \in \mathbb{Z}_{+}$and channels $W_{t}: X_{t} \rightarrow \mathcal{P}\left(\mathcal{Y}_{t}\right)$ for $t \in\{1, \ldots, n\}$, the length $n$ product channel $W_{[1, n]}: \mathcal{X}_{1}^{n} \rightarrow \mathcal{P}\left(\mathcal{Y}_{1}^{n}\right)$ is defined via the following relation:

$$
W_{[1, n]}\left(x_{1}^{n}\right)=\bigotimes_{t=1}^{n} W_{t}\left(x_{t}\right) \quad \forall x_{1}^{n} \in X_{1}^{n}
$$

A channel $U: Z \rightarrow \mathcal{P}\left(\mathcal{Y}_{1}^{n}\right)$ is called a length $n$ memoryless channel iff there exists a product channel $W_{[1, n]}$ satisfying both $U(z)=W_{[1, n]}(z)$ for all $z \in Z$ and $z \subset X_{1}^{n}$. A product channel is stationary iff $W_{t}=W$ for all $t \in\{1, \ldots, n\}$ for some 
$W: X \rightarrow \mathcal{P}(\mathcal{Y})$. For such a channel, we denote the composition (i.e. the empirical distribution, type) of each $x_{1}^{n} \in X_{1}^{n}$ by $\Upsilon(x)$, where $\Upsilon(x) \in \mathcal{P}(X)$.

For any $\ell \in \mathbb{Z}_{+}$, an $\ell$ dimensional cost function $\rho$ is a function from the input set to $\mathbb{R}^{\ell}$ that is bounded from below, i.e. that is of the form $\rho: X \rightarrow \mathbb{R}_{\geq z}^{\ell}$ for some $z \in \mathbb{R}$. We assume without loss of generality that ${ }^{4}$

$$
\inf _{x \in X} \rho^{\imath}(x) \geq 0 \quad \forall \imath \in\{1, \ldots, \ell\} .
$$

We denote the set of all cost constraints that can be satisfied by some member of $\chi$ by $\Gamma_{\rho}^{e x}$ and the set of all cost constraints that can be satisfied by some member of $\mathcal{P}(X)$ by $\Gamma_{\rho}$ :

$$
\begin{aligned}
& \Gamma_{\rho}^{e x} \triangleq\left\{\varrho \in \mathbb{R}_{\geq 0}^{\ell}: \exists x \in \mathcal{X} \text { s.t. } \rho(x) \leq \varrho\right\}, \\
& \Gamma_{\rho} \triangleq\left\{\varrho \in \mathbb{R}_{\geq 0}^{\ell}: \exists p \in \mathcal{P}(\mathcal{X}) \text { s.t. } \mathbf{E}_{p}[\rho] \leq \varrho\right\} .
\end{aligned}
$$

Then both $\Gamma_{\rho}^{e x}$ and $\Gamma_{\rho}$ have non-empty interiors and $\Gamma_{\rho}$ is the convex hull of $\Gamma_{\rho}^{e x}$, i.e. $\Gamma_{\rho}=\operatorname{ch} \Gamma_{\rho}^{e x}$.

A cost function on a product channel is said to be additive iff it can be written as the sum of cost functions defined on the component channels. Given $W_{t}: X_{t} \rightarrow \mathcal{P}\left(\mathcal{Y}_{t}\right)$ and $\rho_{t}: X_{t} \rightarrow \mathbb{R}_{\geq 0}^{\ell}$ for $t \in\{1, \ldots, n\}$, we denote the resulting additive cost function on $X_{1}^{n}$ for the channel $W_{[1, n]}$ by $\rho_{[1, n]}$, i.e.

$$
\rho_{[1, n]}\left(x_{1}^{n}\right)=\sum_{t=1}^{n} \rho_{t}\left(x_{t}\right) \quad \forall x_{1}^{n} \in X_{1}^{n} .
$$

\subsection{Codes With List Decoding}

The pair $(\Psi, \Theta)$ is an $(M, L)$ channel code on $W: X \rightarrow \mathcal{P}(\mathcal{Y})$ iff

- The encoding function $\Psi$ is a function from the message set $\mathcal{M} \triangleq\{1,2, \ldots, M\}$ to the input set $X$.

- The decoding function $\Theta$ is a measurable function from the output space $(y, \mathcal{Y})$ to the set $\widehat{\mathcal{M}} \triangleq\{\mathcal{L}: \mathcal{L} \subset \mathcal{M}$ and $|\mathcal{L}|=L\}$. Given an $(M, L)$ channel code $(\Psi, \Theta)$ on $W: \mathcal{X} \rightarrow \mathcal{P}(\mathcal{Y})$, the conditional error probability $P_{\mathbf{e}}^{m}$ for $m \in \mathcal{M}$ and the average error probability $P_{\mathbf{e}}$ are defined as

$$
\begin{aligned}
& P_{\mathbf{e}}^{m} \triangleq \mathbf{E}_{W(\Psi(m))}\left[\mathbb{1}_{\{m \notin \Theta(y)\}}\right], \\
& P_{\mathbf{e}} \triangleq \frac{1}{M} \sum_{m \in \mathcal{M}} P_{\mathbf{e}}^{m} .
\end{aligned}
$$

An encoding function $\Psi$, hence the corresponding code, is said to satisfy the cost constraint $\varrho$ iff $\bigvee_{m \in \mathcal{M}} \rho(\Psi(m)) \leq \varrho$. An encoding function $\Psi$, hence the corresponding code, on a stationary product channel is said to satisfy an empirical distribution constraint $\mathcal{A} \subset \mathcal{P}(X)$ iff the composition of all of the codewords are in $\mathcal{A}$, i.e. iff $\Upsilon(\Psi(m)) \in \mathcal{A}$ for all $m \in \mathcal{M}$.

\section{PRELIMINARIES}

The Rényi divergence, tilting, and Augustin's information measures are central to the analysis we present in the following sections. We introduce these concepts in $\$ 2.1$ and $\$ 2.2$, a more detailed discussion can be found in [27], [37]. In \$2.3 we define the SPE and derive widely known properties of it for our general channel model. In $\$ 2.4$ we derive Augustin's variant of Gallager's bound.

\subsection{The Rényi Divergence and Tilting}

Definition 1. For any $\alpha \in \mathbb{R}_{+}$and $w, q \in \mathcal{M}^{+}(\mathcal{Y})$, the order $\alpha$ Rényi divergence between $w$ and $q$ is

$$
D_{\alpha}(w \| q) \triangleq \begin{cases}\frac{1}{\alpha-1} \ln \int\left(\frac{\mathrm{d} w}{\mathrm{~d} \nu}\right)^{\alpha}\left(\frac{\mathrm{d} q}{\mathrm{~d} \nu}\right)^{1-\alpha} \nu(\mathrm{d} y) & \alpha \neq 1 \\ \int \frac{\mathrm{d} w}{\mathrm{~d} \nu}\left[\ln \frac{\mathrm{d} w}{\mathrm{~d} \nu}-\ln \frac{\mathrm{d} q}{\mathrm{~d} \nu}\right] \nu(\mathrm{d} y) & \alpha=1\end{cases}
$$

where $\nu$ is any measure satisfying $w \prec \nu$ and $q \prec \nu$.

For properties of the Rényi divergence, throughout the manuscript, we will refer to the comprehensive study provided by van Erven and Harremoës [37]. Note that the order one Rényi divergence is the Kullback-Leibler divergence. For other orders, the Rényi divergence can be characterized in terms of the Kullback-Leibler divergence, as well, see [37, Thm. 30]. That characterization is related to another key concept for our analysis: the tilted probability measure.

Definition 2. For any $\alpha \in \mathbb{R}_{+}$and $w, q \in \mathcal{P}(\mathcal{Y})$ satisfying $D_{\alpha}(w \| q)<\infty$, the order $\alpha$ tilted probability measure $w_{\alpha}^{q}$ is

$$
\frac{\mathrm{d} w_{\alpha}^{q}}{\mathrm{~d} \nu} \triangleq e^{(1-\alpha) D_{\alpha}(w \| q)}\left(\frac{\mathrm{d} w}{\mathrm{~d} \nu}\right)^{\alpha}\left(\frac{\mathrm{d} q}{\mathrm{~d} \nu}\right)^{1-\alpha} .
$$

The conditional Rényi divergence and the tilted channel are straight forward generalizations of the Rényi divergence and the tilted probability measure that will allow us to express certain relations succinctly throughout our analysis.

\footnotetext{
${ }^{4}$ Augustin $[25, \S 33]$ has an additional hypothesis, $\bigvee_{x \in X} \rho(x) \leq \mathbb{1}$, which excludes certain important cases such as the Gaussian channels.
} 
Definition 3. For any $\alpha \in \mathbb{R}_{+}, W: X \rightarrow \mathcal{P}(\mathcal{Y}), Q: X \rightarrow \mathcal{P}(\mathcal{Y})$, and $p \in \mathcal{P}(\mathcal{X})$ the order $\alpha$ conditional Rényi divergence for the input distribution $p$ is

$$
D_{\alpha}(W \| Q \mid p) \triangleq \sum_{x \in X} p(x) D_{\alpha}(W(x) \| Q(x)) .
$$

If $\exists q \in \mathcal{P}(\mathcal{Y})$ such that $Q(x)=q$ for all $x \in \mathcal{X}$, then we denote $D_{\alpha}(W \| Q \mid p)$ by $D_{\alpha}(W \| q \mid p)$.

Definition 4. For any $\alpha \in \mathbb{R}_{+}, W: X \rightarrow \mathcal{P}(\mathcal{Y})$ and $Q: X \rightarrow \mathcal{P}(\mathcal{Y})$, the order $\alpha$ tilted channel $W_{\alpha}^{Q}$ is a function from $\left\{x: D_{\alpha}(W(x) \| Q(x))<\infty\right\}$ to $\mathcal{P}(\mathcal{Y})$ given by

$$
\frac{\mathrm{d} W_{\alpha}^{Q}(x)}{\mathrm{d} \nu} \triangleq e^{(1-\alpha) D_{\alpha}(W(x) \| Q(x))}\left(\frac{\mathrm{d} W(x)}{\mathrm{d} \nu}\right)^{\alpha}\left(\frac{\mathrm{d} Q(x)}{\mathrm{d} \nu}\right)^{1-\alpha} .
$$

If $\exists q \in \mathcal{P}(\mathcal{Y})$ such that $Q(x)=q$ for all $x \in \mathcal{X}$, then we denote $W_{\alpha}^{Q}$ by $W_{\alpha}^{q}$.

The following operator $\mathrm{T}_{\alpha, p}(\cdot)$ was considered implicitly by Fano [9, Ch 9], Haroutunian [10], and Polytrev [31] and explicitly by Augustin [25, §34], but only for orders less than one in all four manuscripts.

Definition 5. For any $\alpha \in \mathbb{R}_{+}, W: \mathcal{X} \rightarrow \mathcal{P}(\mathcal{Y})$, and $p \in \mathcal{P}(\mathcal{X})$, the order $\alpha$ Augustin operator for the input distribution $p$, i.e. $\mathrm{T}_{\alpha, p}(\cdot): \mathcal{Q}_{\alpha, p} \rightarrow \mathcal{P}(\mathcal{Y})$, is given by

$$
\mathrm{T}_{\alpha, p}(q) \triangleq \sum_{x} p(x) W_{\alpha}^{q}(x) \quad \forall q \in \mathcal{Q}_{\alpha, p}
$$

where $\mathcal{Q}_{\alpha, p} \triangleq\left\{q \in \mathcal{P}(\mathcal{Y}): D_{\alpha}(W \| q \mid p)<\infty\right\}$ and the tilted channel $W_{\alpha}^{q}$ is defined in (3).

\subsection{Augustin's Information Measures}

Definition 6. For any $\alpha \in \mathbb{R}_{+}, W: \mathcal{X} \rightarrow \mathcal{P}(\mathcal{Y})$, and $p \in \mathcal{P}(X)$ the order $\alpha$ Augustin information for the input distribution $p$ is

$$
I_{\alpha}(p ; W) \triangleq \inf _{q \in \mathcal{P}(\mathcal{Y})} D_{\alpha}(W \| q \mid p) .
$$

The infimum in (5) is achieved by a unique probability measure denoted by $q_{\alpha, p}$ and called the order $\alpha$ Augustin mean for the input distribution $p$. Furthermore, the order $\alpha$ Augustin mean satisfies the following identities:

$$
\begin{aligned}
D_{1 \vee \alpha}\left(q_{\alpha, p} \| q\right) \geq D_{\alpha}(W \| q \mid p)-I_{\alpha}(p ; W) & \geq D_{1 \wedge \alpha}\left(q_{\alpha, p} \| q\right) & & \forall q \in \mathcal{P}(\mathcal{Y}), \alpha \in \mathbb{R}_{+} . \\
\mathrm{T}_{\alpha, p}\left(q_{\alpha, p}\right) & =q_{\alpha, p} & & \forall \alpha \in \mathbb{R}_{+} .
\end{aligned}
$$

These observations are established in [27, Lemma13-(b,c,d)]; previously they were reported by Augustin [25, Lemma 34.2] for orders less than one. Throughout the manuscript, we refer to [27] for propositions about Augustin's information measures. A more detailed account of the previous work on Augustin's information measures can be found in [27], as well.

Definition 7. For any $\alpha \in \mathbb{R}_{+}, W: \mathcal{X} \rightarrow \mathcal{P}(\mathcal{Y})$, and $\mathcal{A} \subset \mathcal{P}(\mathcal{X})$, the order $\alpha$ Augustin capacity of $W$ for the constraint set $\mathcal{A}$ is

$$
C_{\alpha, W, \mathcal{A}} \triangleq \sup _{p \in \mathcal{A}} I_{\alpha}(p ; W) \text {. }
$$

When the constraint set $\mathcal{A}$ is the whole $\mathcal{P}(X)$, we denote the order $\alpha$ Augustin capacity by $C_{\alpha, W}$, i.e. $C_{\alpha, W} \triangleq C_{\alpha, W, \mathcal{P}(X)}$.

Using the definitions of the Augustin information and capacity we get the following expression for $C_{\alpha, W, \mathcal{A}}$

$$
C_{\alpha, W, \mathcal{A}}=\sup _{p \in \mathcal{A}} \inf _{q \in \mathcal{P}(\mathcal{Y})} D_{\alpha}(W \| q \mid p) .
$$

If $\mathcal{A}$ is convex then the order of the supremum and the infimum can be changed as a result of [27, Thm. 1]:

$$
\sup _{p \in \mathcal{A}} \inf _{q \in \mathcal{P}(\mathcal{Y})} D_{\alpha}(W \| q \mid p)=\inf _{q \in \mathcal{P}(\mathcal{Y})} \sup _{p \in \mathcal{A}} D_{\alpha}(W \| q \mid p) .
$$

If in addition $C_{\alpha, W, \mathcal{A}}$ is finite, then [27, Thm. 1] implies that there exists a unique probability measure $q_{\alpha, W, \mathcal{A}}$, called the order $\alpha$ Augustin center of $W$ for the constraint set $\mathcal{A}$, satisfying

$$
C_{\alpha, W, \mathcal{A}}=\sup _{p \in \mathcal{A}} D_{\alpha}\left(W \| q_{\alpha, W, \mathcal{A}} \mid p\right) .
$$

We denote the set of all probability mass functions satisfying a cost constraint $\varrho$ by $\mathcal{A}(\varrho)$, i.e.

$$
\mathcal{A}(\varrho) \triangleq\left\{p \in \mathcal{P}(X): \mathbf{E}_{p}[\rho] \leq \varrho\right\}
$$

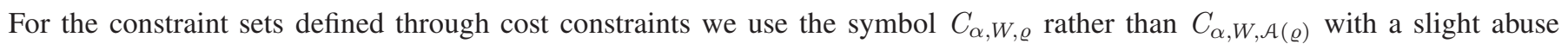
of notation. In order to be able apply convex conjugation techniques without any significant modifications, we extend the definition Augustin capacity to the infeasible cost constraints, i.e. $\varrho$ 's outside $\Gamma_{\rho}$, as follows:

$$
C_{\alpha, W, \varrho} \triangleq\left\{\begin{array}{ll}
\sup _{p \in \mathcal{A}(\varrho)} I_{\alpha}(p ; W) & \text { if } \varrho \in \Gamma_{\rho} \\
-\infty & \text { if } \varrho \in \mathbb{R}_{\geq 0}^{\ell} \backslash \Gamma_{\rho}
\end{array} \quad \forall \alpha \in \mathbb{R}_{+}\right.
$$


In order to characterize $C_{\alpha, W, \varrho}$ through convex conjugation techniques, we first define Augustin-Legendre (A-L) information and capacity. These concepts are first introduced in [1, §III-A] and [27, §5.2], as an extension of the analogous concepts in [12, Ch. 8].

Definition 8. For any $\alpha \in \mathbb{R}_{+}$, channel $W$ of the form $W: X \rightarrow \mathcal{P}(\mathcal{Y})$ with a cost function $\rho: \mathcal{X} \rightarrow \mathbb{R}_{\geq 0}^{\ell}, p \in \mathcal{P}(\mathcal{X})$, and $\lambda \in \mathbb{R}_{\geq 0}^{\ell}$, the order $\alpha$ Augustin-Legendre information for the input distribution $p$ and the Lagrange multiplier $\lambda$ is

$$
I_{\alpha}^{\lambda}(p ; W) \triangleq I_{\alpha}(p ; W)-\lambda \cdot \mathbf{E}_{p}[\rho] .
$$

Definition 9. For any $\alpha \in \mathbb{R}_{+}$, channel $W$ of the form $W: \mathcal{X} \rightarrow \mathcal{P}(\mathcal{Y})$ with a cost function $\rho: \mathcal{X} \rightarrow \mathbb{R}_{\geq 0}^{\ell}$, and $\lambda \in \mathbb{R}_{\geq 0}^{\ell}$ the order $\alpha$ Augustin-Legendre (A-L) capacity for the Lagrange multiplier $\lambda$ is

$$
C_{\alpha, W}^{\lambda} \triangleq \sup _{p \in \mathcal{P}(X)} I_{\alpha}^{\lambda}(p ; W) .
$$

Except for certain sign changes, $C_{\alpha, W}^{\lambda}$ is the convex conjugate of $C_{\alpha, W, \varrho}$ because of an analogous relation between $I_{\alpha}^{\lambda}(p ; W)$ and $I_{\alpha}(p ; W)$, see $[27,(72)-(74),(76)]$.

$$
C_{\alpha, W}^{\lambda}=\sup _{\varrho \geq 0} C_{\alpha, W, \varrho}-\lambda \cdot \varrho \quad \forall \lambda \in \mathbb{R}_{\geq 0}^{\ell} .
$$

Then $C_{\alpha, W, \varrho}$ can be expressed in terms of $C_{\alpha, W}^{\lambda}$ at least for the interior points of $\Gamma_{\rho}$ :

$$
C_{\alpha, W, \varrho}=\inf _{\lambda \geq 0} C_{\alpha, W}^{\lambda}+\lambda \cdot \varrho .
$$

Furthermore, there exists a non-empty convex compact set of $\lambda_{\alpha, W, \varrho}$ 's satisfying $C_{\alpha, W, \varrho}=C_{\alpha, W}^{\lambda_{\alpha, W, \varrho}}+\lambda_{\alpha, W, \varrho} \cdot \varrho$ provided that $C_{\alpha, W, \varrho}$ is finite, by [27, Lemma 29].

On the other hand, using the definitions of $I_{\alpha}(p ; W), I_{\alpha}^{\lambda}(p ; W)$, and $C_{\alpha, W}^{\lambda}$ we get the following expression for $C_{\alpha, W}^{\lambda}$.

$$
C_{\alpha, W}^{\lambda}=\sup _{p \in \mathcal{P}(X)} \inf _{q \in \mathcal{P}(\mathcal{Y})} D_{\alpha}(W \| q \mid p)-\lambda \cdot \mathbf{E}_{p}[\rho] .
$$

$C_{\alpha, W}^{\lambda}$ satisfies a minimax relation similar to the one given in (8), see [27, Thm. 2]. That minimax relation, however, is best understood via the concept of Augustin-Legendre radius defined in the following.

Definition 10. For any $\alpha \in \mathbb{R}_{+}$, channel $W: X \rightarrow \mathcal{P}(\mathcal{Y})$ with a cost function $\rho: X \rightarrow \mathbb{R}_{\geq 0}^{\ell}$, and $\lambda \in \mathbb{R}_{\geq 0}^{\ell}$, the order $\alpha$ Augustin-Legendre radius of $W$ for the Lagrange multiplier $\lambda$ is

$$
S_{\alpha, W}^{\lambda} \triangleq \inf _{q \in \mathcal{P}(\mathcal{Y})} \sup _{x \in \mathcal{X}} D_{\alpha}(W(x) \| q)-\lambda \cdot \rho(x) .
$$

Then as a result of [27, Thm. 2], for any $\alpha \in \mathbb{R}_{+}, W: \mathcal{X} \rightarrow \mathcal{P}(\mathcal{Y})$ with $\rho: \mathcal{X} \rightarrow \mathbb{R}_{\geq 0}^{\ell}$, and $\lambda \in \mathbb{R}_{\geq 0}^{\ell}$ we have

$$
C_{\alpha, W}^{\lambda}=S_{\alpha, W}^{\lambda}
$$

If in addition $C_{\alpha, W}^{\lambda}$ is finite, then there exits a unique $q_{\alpha, W}^{\lambda} \in \mathcal{P}(\mathcal{Y})$, called the order $\alpha$ Augustin-Legendre center of $W$ for the Lagrange multiplier $\lambda$, satisfying

$$
C_{\alpha, W}^{\lambda}=\sup _{x \in X} D_{\alpha}\left(W(x) \| q_{\alpha, W}^{\lambda}\right)-\lambda \cdot \rho(x) .
$$

The A-L information measures are defined through a standard application of the convex conjugation techniques. However, starting with [30, Thms. 8 and 10] - i.e. the cost constrained variants of Gallager's bound- the Rényi-Gallager (R-G) information measures rather than the A-L information measures have been the customary tools for applying convex conjugation techniques in the error exponent calculations, see for example [18]-[20]. A brief discussion of the R-G information information measures can be found in Appendix B; for a more detailed discussion see [27].

\subsection{The Sphere Packing Exponent}

Definition 11. For any $W: \mathcal{X} \rightarrow \mathcal{P}(\mathcal{Y}), \mathcal{A} \subset \mathcal{P}(\mathcal{X})$, and $R \in \mathbb{R}_{\geq 0}$, the SPE is

$$
E_{s p}(R, W, \mathcal{A}) \triangleq \sup _{\alpha \in(0,1)} \frac{1-\alpha}{\alpha}\left(C_{\alpha, W, \mathcal{A}}-R\right) .
$$

We denote $\mathcal{A}=\mathcal{P}(\mathcal{X})$ case by $E_{s p}(R, W)$. Furthermore, with a slight abuse of notation, we denote $\mathcal{A}=\{p\}$ case by $E_{s p}(R, W, p)$ and $\mathcal{A}=\left\{p: \mathbf{E}_{p}[\rho] \leq \varrho\right\}$ case by $E_{s p}(R, W, \varrho)$.

Lemma 1. For any $W: \mathcal{X} \rightarrow \mathcal{P}(\mathcal{Y}), \mathcal{A} \subset \mathcal{P}(\mathcal{X}), E_{s p}(R, W, \mathcal{A})$ is nonincreasing and convex in $R$ on $\mathbb{R}_{\geq 0}$, finite on $\left(C_{0+}, W, \mathcal{A}, \infty\right)$, and continuous on $\left[C_{0+, W, \mathcal{A}}, \infty\right)$ where $C_{0^{+}, W, \mathcal{A}}=\lim _{\alpha \downarrow 0} C_{\alpha, W, \mathcal{A}}$. In particular,

$$
E_{s p}(R, W, \mathcal{A})=\left\{\begin{array}{ll}
\infty & R<C_{0^{+}, W, \mathcal{A}} \\
\sup _{\alpha \in(0,1)} \frac{1-\alpha}{\alpha}\left(C_{\alpha, W, \mathcal{A}}-R\right) & R=C_{0^{+}, W, \mathcal{A}} \\
\sup _{\alpha \in[\phi, 1)} \frac{1-\alpha}{\alpha}\left(C_{\alpha, W, \mathcal{A}}-R\right) & R=C_{\phi, W, \mathcal{A}} \text { for some } \phi \in(0,1) \\
0 & R \geq C_{1, W, \mathcal{A}}
\end{array} .\right.
$$


Lemma 1 follows from the continuity and the monotonicity properties of $C_{\alpha, W, \mathcal{A}}$ established in [27, Lemma 23]; a proof can be found in Appendix C. The proof of Lemma 1 is analogous to that of [26, Lemma 13], which relies on [26, Lemma 8] instead of [27, Lemma 23].

One can express $E_{s p}(R, W, \mathcal{A})$ in terms of $E_{s p}(R, W, p)$, using the definitions of $C_{\alpha, W, \mathcal{A}}, E_{s p}(R, W, \mathcal{A})$, and $E_{s p}(R, W, p)$ :

$$
\begin{aligned}
E_{s p}(R, W, \mathcal{A}) & =\sup _{\alpha \in(0,1)} \sup _{p \in \mathcal{A}} \frac{1-\alpha}{\alpha}\left(I_{\alpha}(p ; W)-R\right) \\
& =\sup _{p \in \mathcal{A}} \sup _{\alpha \in(0,1)} \frac{1-\alpha}{\alpha}\left(I_{\alpha}(p ; W)-R\right) \\
& =\sup _{p \in \mathcal{A}} E_{s p}(R, W, p) .
\end{aligned}
$$

Lemma 1 holds for $E_{s p}(R, W, p)$ by definition, but it can be strengthened significantly for $R$ 's in $\left(\lim _{\alpha \downarrow 0} I_{\alpha}(p ; W), I_{1}(p ; W)\right]$ using the elementary properties of the Augustin information.

Lemma 2. Let $W: \mathcal{X} \rightarrow \mathcal{P}(\mathcal{Y})$ and $p \in \mathcal{P}(\mathcal{X})$ be such that $I_{0^{+}}(p ; W) \neq I_{1}(p ; W)$, where $I_{0^{+}}(p ; W) \triangleq \lim _{\alpha \downarrow 0} I_{\alpha}(p ; W)$. Then for any rate $R \in\left(I_{0+}(p ; W), I_{1}(p ; W)\right]$ there exists a unique order $\alpha^{*} \in(0,1]$ satisfying

$$
R=I_{1}\left(p ; W_{\alpha^{*}}^{q_{\alpha^{*}, p}}\right) .
$$

The orders $\alpha^{*}$ determined by (13) form an increasing continuous bijective function of rate $R$, from $\left(I_{0+}(p ; W), I_{1}(p ; W)\right]$ to $(0,1]$ satisfying

$$
\begin{aligned}
E_{s p}(R, W, p) & =D_{1}\left(W_{\alpha^{*}}^{q_{\alpha^{*}, p}} \| W \mid p\right), \\
\frac{\partial}{\partial R} E_{s p}(R, W, p) & =\frac{\alpha^{*}-1}{\alpha^{*}} .
\end{aligned}
$$

Thus $E_{s p}(R, W, p)$ is finite, convex, continuously differentiable, and decreasing in $R$ on $\left(I_{0+}(p ; W), I_{1}(p ; W)\right)$ and its satisfies

$$
E_{s p}\left(I_{0+}(p ; W), W, p\right)=\lim _{\alpha \downarrow 0} D_{1}\left(W_{\alpha}^{q_{\alpha, p}} \| W \mid p\right) .
$$

Furthermore, if $E_{s p}\left(I_{0+}(p ; W), W, p\right)$ is finite then there exists a $V: \mathcal{X} \rightarrow \mathcal{P}(\mathcal{Y})$ satisfying both $I_{1}(p ; V)=I_{0+}(p ; W)$ and $D_{1}(V \| W \mid p)=E_{s p}\left(I_{0+}(p ; W), W, p\right)$.

Proof of Lemma 2. Note that $I_{1}\left(p ; W_{\alpha}^{q_{\alpha, p}}\right)$ is an increasing and continuous function of the order $\alpha$ by [27, Lemma 17-(a,f)] because $I_{0+}(p ; W) \neq I_{1}(p ; W)$ by the hypothesis. In addition $\lim _{\alpha \downarrow 0} I_{1}\left(p ; W_{\alpha}^{q_{\alpha, p}}\right)=I_{0+}(p ; W)$ by [27, Lemma 17-(g)]. Then there exists a unique $\alpha^{*}$ satisfying (13) by the intermediate value theorem [38, 4.23]. The function defined by (13) is an increasing continuous bijective function of the rate $R$ from $\left(I_{0+}(p ; W), I_{1}(p ; W)\right]$ to $(0,1]$ because it is the inverse of an increasing continuous bijective function from $(0,1]$ to $\left(I_{0+}(p ; W), I_{1}(p ; W)\right]$, i.e. $\alpha \rightsquigarrow I_{1}\left(p ; W_{\alpha}^{q_{\alpha, p}}\right)$.

On the other hand $I_{\alpha}(p ; W)$ is continuously differentiable in $\alpha$ by [27, Lemma 17-(e)]; then [27, (35) and (46)] imply

$$
\frac{\partial}{\partial \alpha} \frac{1-\alpha}{\alpha}\left(I_{\alpha}(p ; W)-R\right)=\frac{1}{\alpha^{2}}\left(R-I_{1}\left(p ; W_{\alpha}^{q_{\alpha, p}}\right)\right) .
$$

Hence for any $R \in\left(I_{0+}(p ; W), I_{1}(p ; W)\right]$, the supremum in the definition of $E_{s p}(R, W, p)$ is achieved at the order $\alpha^{*}$ satisfying (13). Then (14) follows from [27, (35)]. Furthermore, for any $R \in\left(I_{0^{+}}(p ; W), I_{1}(p ; W)\right)$ and $\bar{R} \geq 0$ we have

$$
\begin{aligned}
E_{s p}(\bar{R}, W, p) & \geq \frac{1-\alpha^{*}(R)}{\alpha^{*}(R)}\left(I_{\alpha^{*}}(p ; W)-\bar{R}\right) \\
& =E_{s p}(R, W, p)+\frac{1-\alpha^{*}(R)}{\alpha^{*}(R)}(R-\bar{R}) .
\end{aligned}
$$

For any $\bar{R} \in\left(I_{0^{+}}(p ; W), I_{1}(p ; W)\right)$ and $R \geq 0$, following a similar analysis and reversing the roles of $R$ and $\bar{R}$ we obtain

$$
E_{s p}(R, W, p) \geq E_{s p}(\bar{R}, W, p)+\frac{1-\alpha^{*}(\bar{R})}{\alpha^{*}(\bar{R})}(\bar{R}-R) .
$$

Since $\alpha^{*}$ is increasing and continuous in the rate, (18) and (19) imply (15) for all $R$ 's in $\left(I_{0^{+}}(p ; W), I_{1}(p ; W)\right]$.

For $R=I_{0+}(p ; W)$ case, note that $\frac{1-\alpha}{\alpha}\left(I_{\alpha}(p ; W)-R\right)$ is decreasing $\alpha$ on $(0,1)$ by (17) and [27, Lemma 17-(f,g)]. Thus

$$
E_{s p}\left(I_{0+}(p ; W), W, p\right)=\lim _{\alpha \downarrow 0} \frac{1-\alpha}{\alpha}\left(I_{\alpha}(p ; W)-I_{0+}(p ; W)\right) .
$$

Then (16) follows from the mean value theorem [38, 5.10] and [27, (46)]. Furthermore, if $E_{s p}\left(I_{0+}(p ; W), W, p\right)=\gamma$ for a $\gamma \in \mathbb{R}_{+}$, then $D_{1}\left(W_{\alpha}^{q_{\alpha, p}}(x) \| W(x)\right) \leq \frac{\gamma}{p(x)}$ as a result of non-negativity of the Rényi divergence. Hence

$$
\int G\left(\frac{\mathrm{d} W_{\alpha}^{q \alpha, p}(x)}{\mathrm{d} W(x)}\right) W(\mathrm{~d} y \mid x) \leq \frac{\gamma}{p(x)}+\frac{1}{e}+1
$$

for $G(\tau)=\tau \mathbb{1}_{\{0 \leq \tau<e\}}+\tau \ln \tau \mathbb{1}_{\{\tau \geq e\}}$ because $\tau \ln \tau \geq-1 / e$. Then $\left\{\frac{\mathrm{d} W_{\alpha}^{q_{\alpha}, p}(x)}{\mathrm{d} W(x)}\right\}_{\alpha \in(0,1)}$ are uniformly $W(x)$-integrable by [39, Thm 4.5.9], i.e. by the necessary and sufficient condition for the uniform integrability determined by de la Vallée Poussin. Thus any sequence of members of $\left\{W_{\alpha}^{q_{\alpha, p}}(x)\right\}_{\alpha \in(0,1)}$ has a convergent subsequence for the topology of setwise convergence by [39, Thm. 4.7.25]. For each $x \in \operatorname{supp}(p)$, let $V(x)$ be the limit point for the aforementioned subsequence for the sequence $\left\{W_{1 / \kappa}^{q_{1 / \kappa, p}}(x)\right\}_{\kappa \in \mathbb{Z}_{+}}$. Then (13), (14), and the lower semicontinuity of the Rényi divergence in its arguments for the topology 
of setwise convergence, i.e. [37, Thm. 15], imply $I_{1}(p ; V) \leq I_{0^{+}}(p ; W)$ and $D_{1}(V \| W \mid p) \leq \gamma$. On the other hand as a result of the definition of $E_{s p}(R, W, p)$ and [27, Lemma 13-(e)], we have

$$
\begin{aligned}
E_{s p}(R, W, p) & =\sup _{\alpha \in(0,1)} \inf _{V \in \mathcal{P}(\mathcal{Y} \mid x)} D_{1}(V \| W \mid p)+\frac{1-\alpha}{\alpha}\left(I_{1}(p ; V)-R\right) \\
& \leq \sup _{\alpha \in(0,1)} D_{1}(V \| W \mid p)+\frac{1-\alpha}{\alpha}\left(I_{1}(p ; V)-R\right) \\
& = \begin{cases}D_{1}(V \| W \mid p) & R \geq I_{1}(p ; V) \\
\infty & R<I_{1}(p ; V)\end{cases}
\end{aligned}
$$

Thus $I_{1}(p ; V)$ cannot be less than $I_{0+}(p ; W)$ because $E_{s p}(R, W, p)$ is infinite for all $R<I_{0+}(p ; W)$. Hence $I_{1}(p ; V)=I_{0+}(p ; W)$. Consequently $D_{1}(V \| W \mid p)$ cannot be less than $\gamma$ because $E_{s p}\left(I_{0^{+}}(p ; W), W, p\right)=\gamma$. Hence $D_{1}(V \| W \mid p)=\gamma$.

Lemma 2 provides a simple confirmation of the alternative expression for $E_{s p}(R, W, p)$, which is commonly known as Haroutunian's form [10].

Lemma 3. For any $W: X \rightarrow \mathcal{P}(\mathcal{Y}), p \in \mathcal{P}(\mathcal{X})$, and $R \in \mathbb{R}_{+}$

$$
E_{s p}(R, W, p)=\inf _{V: I_{1}(p ; V) \leq R} D_{1}(V \| W \mid p) .
$$

Proof of Lemma 3. If $R \in\left[I_{1}(p ; W), \infty\right)$, then (20) holds because the expression on the right hand side of (20) is zero as a result of the substitution $V=W$ and the non-negativity of the Rényi divergence.

On the other hand, as a result of the definition of $E_{s p}(R, W, p),[27$, Lemma 13-(e)], and the max-min inequality we have

$$
\begin{aligned}
& E_{s p}(R, W, p)=\sup _{\alpha \in(0,1)} \inf _{V \in \mathcal{P}(\mathcal{Y} \mid x)} D_{1}(V \| W \mid p)+\frac{1-\alpha}{\alpha}\left(I_{1}(p ; V)-R\right) . \\
& \leq \inf _{V \in \mathcal{P}(\mathcal{Y} \mid x)} \sup _{\alpha \in(0,1)} D_{1}(V \| W \mid p)+\frac{1-\alpha}{\alpha}\left(I_{1}(p ; V)-R\right) \\
& =\inf _{V: I_{1}(p ; V) \leq R} D_{1}(V \| W \mid p) .
\end{aligned}
$$

Then (20) holds whenever $E_{s p}(R, W, p)$ is infinite, i.e. for all $R \in\left[0, I_{0+}(p ; W)\right)$ and possibly for $R=I_{0+}(p ; W)$, trivially and whenever $E_{s p}(R, W, p)$ is finite as a result of Lemma 2.

Haroutunian's form implies the following sufficient condition for the optimality of an order $\alpha$ in the definition of the SPE given in (10).

Lemma 4. For any $W: \mathcal{X} \rightarrow \mathcal{P}(\mathcal{Y}), \mathcal{A} \subset \mathcal{P}(\mathcal{X})$, and $R \in\left(C_{0^{+}, W, \mathcal{A}}, C_{1, W, \mathcal{A}}\right)$ if there exists an $\alpha^{*} \in(0,1)$ and a function $V_{p}$ of $p$ from $\mathcal{A}$ to $\mathcal{P}(\mathcal{Y} \mid X)$ satisfying the following two inequalities

$$
\begin{aligned}
D_{1}\left(V_{p} \| q_{\alpha^{*}, W, \mathcal{A}} \mid p\right) & \leq R & & \forall p \in \mathcal{A}, \\
D_{1}\left(V_{p} \| W \mid p\right) & \leq \frac{1-\alpha^{*}}{\alpha^{*}}\left(C_{\alpha^{*}, W, \mathcal{A}}-R\right) & & \forall p \in \mathcal{A},
\end{aligned}
$$

then $E_{s p}(R, W, \mathcal{A})=\frac{1-\alpha^{*}}{\alpha^{*}}\left(C_{\alpha^{*}, W, \mathcal{A}}-R\right)$.

For some channels, $V_{p}=W_{\alpha^{*}}^{q_{\alpha^{*}, W, \mathcal{A}}}$ satisfies both (21) and (22); for these channels, the value of SPE can be determined using Lemma 4. However, for an arbitrary channel, rate, and the corresponding optimal order $\alpha^{*}$ in (10) a $V_{p}$ satisfying both (21) and (22) might not exist, e.g. the $Z$-channel discussed in Appendix A. Had the sufficient condition for the optimality of the order $\alpha^{*}$ given in (21) and (22) been also necessary, Blahut's proof in [32] would have been correct; this, however, is not the case in general as we demonstrate in Appendix A. It is worth mentioning that for the channels satisfying the necessary conditions given (21) and (22), one can derive the SPB using the approach presented in [32].

Proof of Lemma 4. Note that as a result of (6) we have

$$
D_{1}\left(V_{p} \| q_{\alpha^{*}, W, \mathcal{A}} \mid p\right)=I_{1}\left(p ; V_{p}\right)+D_{1}\left(\sum_{x} p(x) V_{p}(x) \| q_{\alpha^{*}, W, \mathcal{A}}\right) .
$$

Thus $I_{1}\left(p ; V_{p}\right) \leq R$ for all $p \in \mathcal{A}$ because the Rényi divergence is non-negative. Then $E_{s p}(R, W, p) \leq \frac{1-\alpha^{*}}{\alpha^{*}}\left(C_{\alpha^{*}, W, \mathcal{A}}-R\right)$ for all $p \in \mathcal{A}$ by Lemma 3. Then $E_{s p}(R, W, \mathcal{A}) \leq \frac{1-\alpha^{*}}{\alpha^{*}}\left(C_{\alpha^{*}, W, \mathcal{A}}-R\right)$ by $(12)$. On the other hand $E_{s p}(R, W, \mathcal{A}) \geq \frac{1-\alpha^{*}}{\alpha^{*}}\left(C_{\alpha^{*}}, W, \mathcal{A}-R\right)$ by definition. Thus $E_{s p}(R, W, \mathcal{A})=\frac{1-\alpha^{*}}{\alpha^{*}}\left(C_{\alpha^{*}, W, \mathcal{A}}-R\right)$.

\subsection{Augustin's Variant of Gallager's Bound}

The SPE is an upper bound on the exponential decay rate of the optimal error probability with block length, i.e. on the reliability function, for memoryless channels satisfying rather mild hypotheses, with or without the list decoding, as a result of the SPBs given Theorem 1 and 2 of $\S 3$. For the list decoding, the SPE is also a lower bound on the exponential decay rate of the optimal error probability with block length [8, ex 5.20], [12, ex 10.28], [40]. The latter observation can be confirmed using standard results such as [30], [31], albeit with minor modifications, as well. In the following, we confirm this observation using a variant of Gallager's bound in terms of the Augustin information. Recall that Gallager's bound is derived, customarily, 
for the Rényi information, rather than the Augustin information. The fixed point property described in (7) plays a critical role in the proof. We name this variant of Gallager's bound after Augustin because [25, Lemma 36.1] of Augustin is the first achievability result making use of the fixed point property described in (7), to the best of out knowledge.

Lemma 5. For any $M, L \in \mathbb{Z}_{+}$s.t. $L<M, W: X \rightarrow \mathcal{P}(\mathcal{Y}), p \in \mathcal{P}(X), \mathcal{B} \subset \mathcal{X}$, and $\alpha \in\left[\frac{1}{1+L}\right.$, 1$)$ there exists an $(M, L)$ channel code with an encoding function of the form $\Psi: \mathcal{M} \rightarrow \mathcal{B}$ satisfying

$$
\ln P_{\mathbf{e}} \leq \frac{\alpha-1}{\alpha}\left[\tau-\ln \frac{(M-1) e}{L}\right]-\frac{\ln p(\mathcal{B})}{\alpha}
$$

where $\tau=\inf _{x \in \mathcal{B}} D_{\alpha}\left(W(x) \| q_{\alpha, p}\right)$.

We do not present a separate proof for Lemma 5 because Lemma 5 is zero Lagrange multiplier special case of Lemma 6 presented in the following. Before stating Lemma 6 , let us point out an immediate consequence of Lemma 5 for the constant composition codes on the stationary memoryless channels. Recall that for any composition $p$ for the block length $n$, the probability of the set of all composition $p$ sequences (i.e. the probability of $\mathcal{T}_{p, n}$ ) for i.i.d. samples (i.e. $p_{t}=p$ for all $t$ ) satisfies the following identity for some $\xi \in[0,1]$ by $[12$, p. 26]

$$
\left(\bigotimes_{t=1}^{n} p_{t}\right)\left(\mathcal{T}_{p, n}\right)=e^{-\xi \frac{|\operatorname{supp}(p)|}{12 \ln 2}}(2 \pi n)^{-\frac{|\operatorname{supp}(p)|-1}{2}} \sqrt{\prod_{x: p(x)>0} \frac{1}{p(x)}}
$$

Then using $q_{\alpha, \bigotimes_{t=1}^{n} p_{t}}=\bigotimes_{t=1}^{n} q_{\alpha, p_{t}}$, established in [27, Lemma 14], we get the following corollary by setting $\mathcal{B}$ to $\mathcal{T}_{p, n}$.

Corollary 1. For any $n \in \mathbb{Z}_{+}, \phi \in(0,1),\left\{W_{t}\right\}_{t \in \mathbb{Z}_{+}}$satisfying $W_{t}=W$ for some $W: X \rightarrow \mathcal{P}(\mathcal{Y}), p \in \mathcal{P}(X)$ satisfying $n p(x) \in \mathbb{Z}_{\geq 0}$ for all $x \in \mathcal{X}$, integers $M, L$ satisfying both $\frac{1}{L+1}<\eta$ and $\frac{1}{n} \ln \frac{M}{L}=I_{\eta}(p ; W)$ for some $\eta \in[\phi, 1)$, there exists an $(M, L)$ channel code on $\bigotimes_{t=1}^{n} W_{t}$ with an encoding function of the form $\Psi: \mathcal{M} \rightarrow \mathcal{T}_{p, n}$ satisfying

$$
\ln P_{\mathbf{e}} \leq-n E_{s p}\left(\frac{1}{n} \ln \frac{M}{L}, W, p\right)+\frac{1}{\eta}\left[1-\eta+\frac{|\operatorname{supp}(p)|}{12 \ln 2}+\frac{|\operatorname{supp}(p)|-1}{2} \ln (2 \pi n)+\frac{1}{2} \sum_{x: p(x)>0} \ln p(x)\right] .
$$

Lemma 6. For any $\ell, M, L \in \mathbb{Z}_{+}$s.t. $L<M, W: X \rightarrow \mathcal{P}(\mathcal{Y}), \rho: \mathcal{X} \rightarrow \mathbb{R}_{\geq 0}^{\ell}, p \in \mathcal{P}(X), \mathcal{B} \subset \mathcal{X}$, and $\alpha \in\left[\frac{1}{1+L}, 1\right)$ there exists an $(M, L)$ channel code with an encoding function of the form $\Psi: \mathcal{M} \rightarrow \mathcal{B}$ such that

$$
\ln P_{\mathbf{e}} \leq \frac{\alpha-1}{\alpha}\left[\tau+\left(\inf _{x \in \mathcal{B}} \lambda \cdot \rho(x)\right)-\ln \frac{(M-1) e}{L}\right]-\frac{\ln p(\mathcal{B})}{\alpha}
$$

where $\tau=\inf _{x \in \mathcal{B}} D_{\alpha}\left(W(x) \| q_{\alpha, p}\right)-\lambda \cdot \rho(x)$.

It is worth mentioning that one can obtain a similar bound in terms of the R-G information, see Lemma 12 in Appendix B. Before presenting the proof of Lemma 6, let us discuss its consequences briefly. For the cost constrained memoryless channels with additive cost functions one can apply Lemma 6 for $\lambda=0$ and for the product input distributions satisfying the cost constraint $\varrho-\epsilon$. Then using the weak law of large numbers together with $[27$, Lemmas $14,27,28]$ one can conclude that the reliability function is bounded from below by the SPE, under rather mild hypotheses. This observation can be strengthened for $\ell=1$ case by invoking Berry-Esseen theorem via [26, Lemma 19], under appropriate hypotheses. The following corollary can be generalized to $\ell>1$ case in a straightforward way once a result analogous to [26, Lemma 19] for sums of random vectors is obtained.

Corollary 2. Let $n \in \mathbb{Z}_{+}$s.t $n \geq 21, \phi \in(0,1), W_{[1, n]}: X_{1}^{n} \rightarrow \mathcal{P}\left(\mathcal{Y}_{1}^{n}\right)$ be a length $n$ product channel with an additive cost function $\rho_{[1, n]}: X_{1}^{n} \rightarrow \mathbb{R}_{\geq 0}$ satisfying $\inf _{x_{t} \in X_{t}} \rho_{t}\left(x_{t}\right)=0$ for all $t \in[1, n]$ and

$$
C_{\alpha, \widetilde{W}_{[1, n]}, n \varrho} \geq C_{\alpha, W_{[1, n]}, n \varrho}-\epsilon \quad \forall \alpha \in[\phi, 1), \varrho \in\left[\widetilde{\varrho}, \widetilde{\varrho}+\frac{9 \varsigma}{n}\right]
$$

for some $\widetilde{\varrho} \in \mathbb{R}_{+}, \varsigma \in \mathbb{R}_{\geq 0}, \epsilon \in \mathbb{R}_{\geq 0}$, and product channel $\widetilde{W}_{[1, n]}: \widetilde{X}_{1}^{n} \rightarrow \mathcal{P}\left(\mathcal{Y}_{1}^{n}\right)$ with the input sets $\widetilde{X}_{t}=\left\{x_{t} \in X_{t}: \rho_{t}\left(x_{t}\right) \leq \varsigma\right\}$ satisfying $\widetilde{W}_{[1, n]}\left(x_{1}^{n}\right)=W_{[1, n]}\left(x_{1}^{n}\right)$ for all $x_{1}^{n} \in \widetilde{X}_{1}^{n}$. Then for any $\delta \in(0, \widetilde{\varrho}), \varrho \in\left(\widetilde{\varrho}+\frac{3 e \varsigma}{n}, \widetilde{\varrho}+\frac{9 \varsigma}{n}\right)$, and integers $M, L$ satisfying

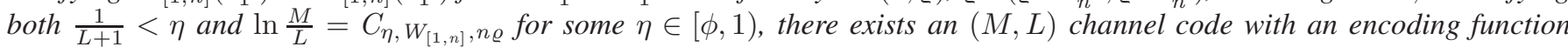
of the form $\Psi: \mathcal{M} \rightarrow\left\{x_{1}^{n} \in \widetilde{X}_{1}^{n}: \rho_{[1, n]}\left(x_{1}^{n}\right) \leq n \varrho\right\}$ such that

$$
\ln P_{\mathbf{e}} \leq-E_{s p}\left(\ln \frac{M}{L}, W_{[1, n]}, n \varrho\right)+\frac{1-\eta}{\eta}\left(\frac{C_{\eta, W_{[1, n]}, n \varrho}}{n} \frac{6 \varsigma e}{\delta}+2 \epsilon+1\right)+\frac{\ln 4 n}{2 \eta}+\frac{1}{n} .
$$

Note that if the region for $\varrho$ had not depended on $\varsigma$ in (24) and the interval for $\alpha$ had been a compact subset of $(0,1)$, then one could have found a $\varsigma$ for any positive $\epsilon$ by invoking Dini's theorem [36, 2.4.10] through a construction similar to the one used in the proof of $[28$, Lemma $15-(\mathrm{g})]$ because $C_{\alpha, W, \varrho}$ is continuous in $(\alpha, \varrho)$ on $(0,1) \times \mathbb{R}_{+}$by [27, Lemma 27-(c)]. This, however, is not the case and there might be product channels for which (24) is not satisfied for any $(\epsilon, \varsigma, \widetilde{\varrho})$ triple. Furthermore, even when the hypotheses of Corollary 2 are satisfied the tightness of the bound given in (25) depend on the values of the constants satisfying the hypotheses. Nevertheless it is easy to see that if either the cost functions of the component channels are all bounded or $C_{\phi, W_{[1, n]}, n \varrho}$ is $\Theta(n)$-i.e. scaling linearly with $n$ - and (24) is satisfied for $\epsilon$ and $\varsigma$ that are $O(\ln n)$ 
-i.e. scaling no faster than linearly with $\ln n$ - then (25) has a prefactor that is $e^{-O(\ln n)}$, i.e. vanishing polynomially with $n$. Proof of Corollary 2 is presented in Appendix C.

Proof of Lemma 6. We establish the existence of the code with the desired properties through a random coding argument. For any $p \in \mathcal{P}(X)$ and $\mathcal{B} \subset \mathcal{X}$ satisfying $p(\mathcal{B})>0$, let $p_{\mathcal{B}} \in \mathcal{P}(\mathcal{X})$ be

$$
p_{\mathcal{B}}(x) \triangleq \frac{\mathbb{1}_{\{x \in \mathcal{B}\}} p(x)}{p(\mathcal{B})} \quad \forall x \in X .
$$

Let us consider an ensemble of codes in which the assignments of the messages to the elements of $\mathcal{B}$ are jointly independent and $\Psi(m)=x$ with probability $p_{\mathcal{B}}(x)$ for all $m$ in the message set. The decoder chooses $L$ messages with the greatest $\frac{f_{\Psi(m)}}{h_{\Psi}(m)}$ for $f_{x}$ and $h_{x}$ defined in (27) and (28) in the following. When there is a tie, the decoder prefers the message or messages with the lower index among the tied messages.

$$
\begin{array}{rlrl}
f_{x}(y) \triangleq \frac{\mathrm{d} W(x)}{\mathrm{d} q}(y) & & \forall x \in X, y \in \mathcal{Y} \\
h_{x} \triangleq e^{\frac{\alpha-1}{\alpha} \lambda \cdot \rho(x)} & \forall x \in X
\end{array}
$$

where $q \in \mathcal{P}(\mathcal{Y})$ to be chosen later satisfies $q_{\alpha, p} \prec q$. In order to bound the expected value of the error probability of the code over the ensemble, let us consider the expected value of the conditional error probability of the message with the greatest index. An error occurs only when $\frac{f}{h}$ associated with $L$ or more other messages are at least as large as $\frac{f}{h}$ associated with the transmitted message. We can bound this probability using a $y$-dependent auxiliary threshold $\gamma(y)$ and obtain the following bound on the expected value of $P_{\mathbf{e}}$ over the ensemble. ${ }^{5}$

$$
\mathbf{E}\left[P_{\mathbf{e}}\right] \leq \sum_{x} p_{\mathcal{B}}(x) \mathbf{E}_{q}\left[\mathbb{1}_{\left\{\frac{f_{x}}{h_{x}} \leq \gamma\right\}} f_{x}\right]+\left(\begin{array}{c}
M-1 \\
L
\end{array}\right) \sum_{x} p_{\mathcal{B}}(x) \mathbf{E}_{q}\left[\mathbb{1}_{\left\{\frac{f_{x}}{h_{x}}>\gamma\right\}}\left[\sum_{z} p_{\mathcal{B}}(z) \mathbb{1}_{\left\{\frac{f_{z}}{h_{z}} \geq \frac{f_{x}}{h_{x}}\right\}}\right]^{L} f_{x}\right]
$$

We invoke $\alpha \leq 1$ to bound the first term in the above sum, and $\alpha \geq \frac{1}{1+L}$ to bound the second term.

$$
\begin{aligned}
\mathbf{E}_{q}\left[\mathbb{1}_{\left\{\frac{f_{x}}{h_{x}} \leq \gamma\right\}} f_{x}\right] & \leq h_{x} \mathbf{E}_{q}\left[\mathbb{1}_{\left\{\frac{f_{x}}{h_{x}} \leq \gamma\right\}}\left(\frac{f_{x}}{h_{x}}\right)^{\alpha} \gamma^{1-\alpha}\right] \\
\mathbf{E}_{q}\left[\mathbb{1}_{\left\{\frac{f_{x}}{h_{x}}>\gamma\right\}}\left[\sum_{z} p_{\mathcal{B}}(z) \mathbb{1}_{\left\{\frac{f_{z}}{h_{z}} \geq \frac{f_{x}}{h_{x}}\right\}}\right]^{L} f_{x}\right] & \leq h_{x} \mathbf{E}_{q}\left[\mathbb{1}_{\left\{\frac{f_{x}}{h_{x}}>\gamma\right\}}\left[\sum_{z} p_{\mathcal{B}}(z)\left(\frac{f_{z}}{h_{z}}\right)^{\alpha}\right]^{L}\left(\frac{f_{x}}{h_{x}}\right)^{1-L \alpha}\right] \\
& \leq h_{x} \mathbf{E}_{q}\left[\mathbb{1}_{\left\{\frac{f_{x}}{h_{x}}>\gamma\right\}}\left[\sum_{z} p_{\mathcal{B}}(z)\left(\frac{f_{z}}{h_{z}}\right)^{\alpha}\right]^{L}\left(\frac{f_{x}}{h_{x}}\right)^{\alpha} \gamma^{1-\alpha-L \alpha}\right]
\end{aligned}
$$

If we set $\gamma=\left[\sum_{x} p_{\mathcal{B}}(x)\left(\frac{f_{x}}{h_{x}}\right)^{\alpha}\right]^{\frac{1}{\alpha}}\left[\left(\begin{array}{c}M-1 \\ L\end{array}\right)\right]^{\frac{1}{L \alpha}}$ we get

$$
\begin{aligned}
\mathbf{E}\left[P_{\mathbf{e}}\right] & \leq \sum_{x} p_{\mathcal{B}}(x) h_{x} \mathbf{E}_{q}\left[\left(\frac{f_{x}}{h_{x}}\right)^{\alpha} \gamma^{1-\alpha}\right] \\
& =\mathbf{E}_{q}\left[\left[\sum_{x} p_{\mathcal{B}}(x)\left(\frac{f_{x}}{h_{x}}\right)^{\alpha}\right]^{\frac{1}{\alpha}}\right]\left(\sup _{x \in \mathcal{B}} h_{x}\right)\left[\left(\begin{array}{c}
M-1 \\
L
\end{array}\right)\right]^{\frac{1-\alpha}{L \alpha}} .
\end{aligned}
$$

On the other hand the Stirling's approximation for the factorials, i.e. $\sqrt{2 \pi n}(n / e)^{n} \leq n ! \leq e \sqrt{n}(n / e)^{n}$, and the identity $\ln z \leq z-1$ imply that

$$
\begin{aligned}
\frac{1}{L} \ln \left(\begin{array}{c}
M-1 \\
L
\end{array}\right) & \leq \frac{1}{L} \ln \frac{e \sqrt{M-1}}{2 \pi \sqrt{L(M-1-L)}}+\ln \frac{M-1}{L}+\frac{M-1-L}{L} \ln \left(1+\frac{L}{M-1-L}\right) \\
& \leq \ln \frac{M-1}{L}+1 .
\end{aligned}
$$

Then we get the following bound on the expected value of $P_{\mathbf{e}}$ over the ensemble.

$$
\ln \mathbf{E}\left[P_{\mathbf{e}}\right] \leq \ln \mathbf{E}_{q}\left[\left(\sum_{x} p_{\mathcal{B}}(x) e^{(1-\alpha) \lambda \cdot \rho(x)}\left(\frac{\mathrm{d} W(x)}{\mathrm{d} q}\right)^{\alpha}\right)^{\frac{1}{\alpha}}\right]+\frac{\alpha-1}{\alpha}\left[\left(\inf _{x \in \mathcal{B}} \lambda \cdot \rho(x)\right)-\ln \frac{(M-1) e}{L}\right]
$$

If we set $q=q_{\alpha, p}$, using first the definition of the tilted channel given in (3) and the definition of $\tau$ we get

$$
\begin{aligned}
\ln \mathbf{E}\left[P_{\mathbf{e}}\right] & \leq \ln \mathbf{E}_{q_{\alpha, p}}\left[\left(\sum_{x} p_{\mathcal{B}}(x) e^{(\alpha-1)\left[D_{\alpha}\left(W(x) \| q_{\alpha, p}\right)-\lambda \cdot \rho(x)\right]} \frac{\mathrm{d} W_{\alpha}^{q_{\alpha}, p}(x)}{\mathrm{d} q_{\alpha, p}}\right)^{\frac{1}{\alpha}}\right]+\frac{\alpha-1}{\alpha}\left[\left(\inf _{x \in \mathcal{B}} \lambda \cdot \rho(x)\right)-\ln \frac{(M-1) e}{L}\right] \\
& \leq \ln \mathbf{E}_{q_{\alpha, p}}\left[\left(\sum_{x} p_{\mathcal{B}}(x) \frac{\mathrm{d} W_{\alpha}^{q_{\alpha, p}}(x)}{\mathrm{d} q_{\alpha, p}}\right)^{\frac{1}{\alpha}}\right]+\frac{\alpha-1}{\alpha}\left[\tau+\left(\inf _{x \in \mathcal{B}} \lambda \cdot \rho(x)\right)-\ln \frac{(M-1) e}{L}\right] .
\end{aligned}
$$

Since there exists a code with $P_{\mathbf{e}}$ less than or equal to $\mathbf{E}\left[P_{\mathbf{e}}\right]$, the existence of a code satisfying (23) with an encoding function of the form $\Psi: \mathcal{M} \rightarrow \mathcal{B}$ follows from (4), (7), and (26).

$$
{ }^{5} \text { Note that } \sum_{t=L}^{M}\left(\begin{array}{c}
M \\
t
\end{array}\right) s^{t}(1-s)^{M-t}=\left(\begin{array}{c}
M \\
L
\end{array}\right) s^{L} \sum_{t=0}^{M-L} \frac{L !(M-L) !}{(L+t) !(M-L-t)} s^{t}(1-s)^{M-L-t} \leq\left(\begin{array}{c}
M \\
L
\end{array}\right) s^{L} \text { for all } s \in[0,1] .
$$




\section{Sphere Packing Bounds For Memoryless Channels}

Assumption 1. The maximum $C_{1 / 2, U_{\mathcal{A}}^{(t)}}$ for integers $t$ less than or equal to $n$ is $O(\ln n)$, i.e.

$$
\exists n_{0} \in \mathbb{Z}_{+}, K \in \mathbb{R}_{+} \quad \text { such that } \quad \max _{t: t \leq n} C_{1 / 2, U_{\mathcal{A}}^{(t)}} \leq K \ln (n) \quad \forall n \geq n_{0},
$$

where $U_{\mathcal{A}}^{(t)}: X_{\mathcal{A}}^{(t)} \rightarrow \mathcal{P}(\mathcal{Y})$ satisfies $U_{\mathcal{A}}^{(t)}(x)=W(x)$ for all $x \in X_{\mathcal{A}}^{(t)}$ and $X_{\mathcal{A}}^{(t)}$ is defined for any $t \in \mathbb{Z}+$ and $\mathcal{A} \subset \mathcal{P}(X)$ as

$$
X_{\mathcal{A}}^{(t)} \triangleq\{x \in \mathcal{X}: \exists p \in \mathcal{A} \text { s.t. } p(z) t \in \mathbb{Z} \forall z \in \mathcal{X} \text { for which } p(x)>0\} .
$$

Theorem 1. Let $\left\{W_{t}\right\}_{t \in \mathbb{Z}_{+}}$be a stationary sequence of channels satisfying $W_{t}=W$ for all $t \in \mathbb{Z}_{+}, \mathcal{A} \subset \mathcal{P}(\mathcal{X})$ be a convex constraint set satisfying Assumption $1, \varepsilon, \alpha_{0}, \alpha_{1}$ be positive parameters satisfying $0<\alpha_{0}<\alpha_{1}<1$. Then for any sequence of codes $\left\{\left(\Psi^{(n)}, \Theta^{(n)}\right)\right\}_{n \in \mathbb{Z}_{+}}$on the product channels $\left\{W_{[1, n]}\right\}_{n \in \mathbb{Z}_{+}}$satisfying

$$
C_{\alpha_{1}, W, \mathcal{A}} \geq \frac{1}{n} \ln \frac{M_{n}}{L_{n}} \geq C_{\alpha_{0}, W, \mathcal{A}}+\frac{\ln n}{n}[\ln (\ln n)]^{\varepsilon} \quad \forall n \geq n_{0}
$$

and $\Upsilon\left(\Psi^{(n)}(m)\right) \in \mathcal{A}$ for all $m \in \mathcal{M}^{(n)}$, there exists $a \tau \in \mathbb{R}_{+}$and an $n_{1} \geq n_{0}$ such that

$$
P_{\mathbf{e}}^{(n)} \geq n^{-\tau} e^{-n E_{s p}\left(\frac{1}{n} \ln \frac{M_{n}}{L_{n}}, W, \mathcal{A}\right)} \quad \forall n \geq n_{1} .
$$

It is worth mentioning that the hypotheses of Theorem 1 are satisfied by the Gaussian and Poisson models considered in [15]-[23]; thus, Theorem 1 implies an asymptotic SPB for these channels. The non-asymptotic counterpart of this implication is presented in $\S 3.3$, between Lemma 9 and Corollary 3.

For codes on the cost constraint memoryless channels one can remove the stationarity hypothesis using the convex conjugation techniques, [25, Thm. 36.6]. Theorem 2 strengthens [25, Thm. 36.6] by removing the bounded cost function hypothesis and by establishing the SPB with a prefactor that is polynomial in the block length $n$ rather than a prefactor of the from $e^{-O(\sqrt{n})}$.

Assumption 2. The maximum $C_{1 / 2}, W_{t}, n \varrho$ for integers $t$ less than or equal to $n$ is $O(\ln n)$.

$$
\exists n_{0} \in \mathbb{Z}_{+}, K \in \mathbb{R}_{+} \quad \text { such that } \quad \max _{t: t \leq n} C_{1 / 2, W_{t}, n \varrho} \leq K \ln (n) \quad \forall n \geq n_{0} .
$$

Theorem 2. Let $\left\{\left(W_{t}, \rho_{t}\right)\right\}_{t \in \mathbb{Z}_{+}}$be a sequence of channels with associated cost functions satisfying Assumption 2 , $\varepsilon$, $\alpha_{0}$, $\alpha_{1}$ be positive parameters satisfying $0<\alpha_{0}<\alpha_{1}<1$. Then on the product channels $\left\{W_{[1, n]}\right\}_{n \in \mathbb{Z}_{+}}$with additive cost functions $\left\{\rho_{[1, n]}\right\}_{n \in \mathbb{Z}_{+}}$of the form $\rho_{[1, n]}\left(x_{1}^{n}\right)=\sum_{t=1}^{n} \rho_{t}\left(x_{t}\right)$, any sequence of codes $\left\{\left(\Psi^{(n)}, \Theta^{(n)}\right)\right\}_{n \in \mathbb{Z}_{+}}$satisfying

$$
C_{\alpha_{1}, W_{[1, n]}, n \varrho} \geq \ln \frac{M_{n}}{L_{n}} \geq C_{\alpha_{0}, W_{[1, n]}, n \varrho}+\varepsilon(\ln n)^{2} \quad \forall n \geq n_{0}
$$

and $\rho_{[1, n]}\left(\Psi^{(n)}(m)\right) \leq n \varrho$ for all $m \in \mathcal{M}^{(n)}$ for a per channel use cost constraint $\varrho$ satisfying $n \varrho \in$ int $\Gamma_{\rho_{[1, n]}}$, there exists a $\tau \in \mathbb{R}_{+}$and an $n_{1} \geq n_{0}$ such that

$$
P_{\mathbf{e}}^{(n)} \geq n^{-\tau} e^{-E_{s p}\left(\ln \frac{M_{n}}{L_{n}}, W_{[1, n]}, n \varrho\right)} \quad \forall n \geq n_{1} .
$$

In order to establish the asymptotic SPB with a polynomial prefactor in Theorem 2 , we have assumed that the order $1 / 2$ cost constrained Augustin capacity is scaling no faster than logarithmically with the cost. Although this hypothesis is true for most cases of interest, including various Gaussian and Poisson channels considered in [15]-[23] and many of their non-stationary variants, there does exist channels violating it, see [27, Example 1]. Thus one might want to remove the logarithmic growth with the cost constraint hypothesis. One can do so by using Lemma 11 instead of Lemma 10 in the proof of Theorem 2.

Our ultimate aim in this section is to prove the two asymptotic SPBs given in Theorems 1 and 2, which constitute the main contribution of this article. To that end, we first provide an impossibility result for the hypothesis testing problem with independent samples using Berry-Esseen theorem in $\$ 3.1$. Then, we introduce the concepts of averaged Augustin capacity and averaged SPE in $\$ 3.2$. We derive non-asymptotic — but parametric — SPBs in terms of these averaged quantities for the composition constrained codes on stationary memoryless channels in $\$ 3.3$ and for the cost constrained codes on (possibly nonstationary) memoryless channels in \$3.4. The derivation of the asymptotic SPBs using the non-asymptotic ones and Lemma 8 of $\S 3.2$, which is rather straightforward, is presented in Appendix C.

\subsection{An Impossibility Result For Hypothesis Testing}

Lemma 7. For any $\kappa \geq 3, \alpha \in(0,1), n \in \mathbb{Z}_{+}$, and product measures $w, q \in \mathcal{P}\left(\mathcal{Y}_{1}^{n}\right)$ of the form $w=\bigotimes_{t=1}^{n} w_{t}$ and $q=\bigotimes_{t=1}^{n} q_{t}$, let $\xi_{t} \triangleq \ln \frac{\mathrm{d} w_{t, a c}}{\mathrm{~d} q_{t}}-\mathbf{E}_{w_{\alpha}^{q}}\left[\ln \frac{\mathrm{d} w_{t, a c}}{\mathrm{~d} q_{t}}\right]$ where $w_{t, a c}$ is the component of $w_{t}$ that is absolutely continuous in $q_{t}$ and

Then any $\mathcal{E} \in \mathcal{Y}$ satisfying $q(\mathcal{E}) \leq \frac{1}{4 \sqrt{n}} e^{-D_{1}\left(w_{\alpha}^{q} \| q\right)-\alpha 3 g_{\kappa}}$ also satisfies

$$
g_{\kappa} \triangleq\left(\sum_{t=1}^{n} \mathbf{E}_{w_{\alpha}^{q}}\left[\left|\xi_{t}\right|^{\kappa}\right]\right)^{1 / \kappa}
$$

$$
w\left(y_{1}^{n} \backslash \mathcal{E}\right) \geq \frac{1}{4 \sqrt{n}} e^{-D_{1}\left(w_{\alpha}^{q} \| w\right)-(1-\alpha) 3 g_{\kappa}} .
$$


Lemma 7 provides an impossibility result for the hypothesis testing problem with independent samples in the spirit of [7, Thm. 5]. It, however, relies on the Berry-Esseen Theorem via [26, Lemma 19] rather than the Chebyshev inequality.

Proof of Lemma 7. Let $\mathcal{E}_{0}=\left\{y:\left|\sum_{t=1}^{n} \xi_{t}\right| \leq 3 g_{\kappa}\right\}$ then

$$
\begin{array}{ll}
\left|\ln \frac{\mathrm{d} w_{\alpha}^{q}}{\mathrm{~d} q}\left(y_{1}^{n}\right)-D_{1}\left(w_{\alpha}^{q} \| q\right)\right| \leq \alpha 3 g_{\kappa} & \forall y_{1}^{n} \in \mathcal{E}_{0}, \\
\left|\ln \frac{\mathrm{d} w_{\alpha}^{q}}{\mathrm{~d} w}\left(y_{1}^{n}\right)-D_{1}\left(w_{\alpha}^{q} \| w\right)\right| \leq(1-\alpha) 3 g_{\kappa} & \forall y_{1}^{n} \in \mathcal{E}_{0} .
\end{array}
$$

Hence,

$$
\begin{aligned}
& w_{\alpha}^{q}\left(\mathcal{E} \cap \mathcal{E}_{0}\right) \leq q\left(\mathcal{E} \cap \mathcal{E}_{0}\right) e^{D_{1}\left(w_{\alpha}^{q} \| q\right)+\alpha 3 g_{\kappa}}, \\
& w_{\alpha}^{q}\left(\mathcal{E} \cap \mathcal{E}_{0}\right) \leq w\left(\mathcal{E} \cap \mathcal{E}_{0}\right) e^{D_{1}\left(w_{\alpha}^{q} \| w\right)+(1-\alpha) 3 g_{\kappa}} .
\end{aligned}
$$

On the other hand as a result of the definition of $\mathcal{E}_{0}$ and [26, Lemma 19] we have

$$
\mathbf{P}_{w_{\alpha}^{q}}\left[\mathcal{E}_{0}\right] \geq \frac{1}{2 \sqrt{n}}
$$

(35) follows from (36), (37), and (38).

\subsection{Augustin's Averaging}

Our non-asymptotic SPBs are expressed in terms of the averaged Augustin capacity and averaged SPE defined in the following for all $\epsilon \in(0,1)$ and $R \in \mathbb{R}_{\geq 0}$.

$$
\begin{aligned}
\widetilde{C}_{\alpha, W, \mathcal{A}}^{\epsilon} \triangleq \frac{1}{\epsilon} \int_{\alpha-\epsilon \alpha}^{\alpha+\epsilon(1-\alpha)}\left[1 \vee\left(\frac{\alpha}{1-\alpha} \frac{1-\eta}{\eta}\right)\right] C_{\eta, W, \mathcal{A}} \mathrm{d} \eta \\
\widetilde{E}_{s p}^{\epsilon}(R, W, \mathcal{A}) \triangleq \sup _{\alpha \in(0,1)} \frac{1-\alpha}{\alpha}\left(\widetilde{C}_{\alpha, W, \mathcal{A}}^{\epsilon}-R\right)
\end{aligned}
$$

Note that $\lim _{\epsilon \downarrow 0} \widetilde{C}_{\alpha, W, \mathcal{A}}^{\epsilon}=C_{\alpha, W, \mathcal{A}}$ for all $\alpha \in(0,1)$ because $C_{\alpha, W, \mathcal{A}}$ is continuous in $\alpha$ on $(0,1)$ by [27, Lemma 23-(d)]. Furthermore, one can show that the convergence is uniform on compact subsets of $(0,1)$ using the monotonicity of $C_{\alpha, W, \mathcal{A}}$

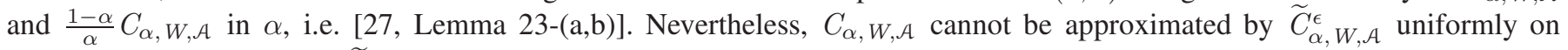
$(0,1)$ itself because $\lim _{\alpha \uparrow 1} \widetilde{C}_{\alpha, W, \mathcal{A}}^{\epsilon}=\infty$ for all positive $\epsilon$ 's whenever $C_{1 / 2, W, \mathcal{A}}$ is positive, even if $C_{1, W, \mathcal{A}}$ is finite. The latter observation follows from the monotonicity of $C_{\alpha, W, \mathcal{A}}$ and $\frac{1-\alpha}{\alpha} C_{\alpha, W, \mathcal{A}}$ in $\alpha$ :

$$
\begin{aligned}
\widetilde{C}_{\alpha, W, \mathcal{A}}^{\epsilon} & \geq \frac{1}{\epsilon} \int_{\alpha-\epsilon \alpha}^{\alpha-\frac{\epsilon}{2} \alpha} \frac{\alpha}{1-\alpha} \frac{1-\eta}{\eta} C_{\eta, W, \mathcal{A}} \mathrm{d} \eta \\
& \geq \frac{\alpha}{2-\epsilon}\left(1+\frac{\alpha \epsilon}{2(1-\alpha)}\right) C_{\alpha-\epsilon \alpha, W, \mathcal{A}} .
\end{aligned}
$$

As a function of the rate, the averaged SPE converges uniformly to the SPE on any compact set of rates less than the order one Augustin capacity, as demonstrated by the following lemma.

Lemma 8. For any $W: X \rightarrow \mathcal{P}(\mathcal{Y}), \mathcal{A} \subset \mathcal{P}(X)$ satisfying $C_{1 / 2, W, \mathcal{A}} \in \mathbb{R}_{+}, \phi \in(0,1), R \in\left[C_{\phi}, W, \mathcal{A}, \infty\right)$, and $\in \in(0, \phi)$,

$$
\begin{aligned}
0 \leq \widetilde{E}_{s p}^{\epsilon}(R, W, \mathcal{A})-E_{s p}(R, W, \mathcal{A}) & \leq \frac{\epsilon}{1-\epsilon} \frac{R \vee E_{s p}(R, W, \mathcal{A})}{\phi} \\
& \leq \frac{\epsilon}{1-\epsilon} \frac{R}{\phi^{2}} .
\end{aligned}
$$

Lemma 8 follows from the monotonicity of $C_{\alpha, W, \mathcal{A}}$ and $\frac{1-\alpha}{\alpha} C_{\alpha, W, \mathcal{A}}$ in $\alpha$ established in [27, Lemma 23-(a,b)]; a proof can be found in Appendix $C$. The proof of Lemma 8 is nearly identical to the proof of [26, Lemma 15], establishing the very same result for $\mathcal{A}=\mathcal{P}(\mathcal{X})$ case.

\subsection{Non-asymptotic Sphere Packing Bounds for the Composition Constrained Codes}

The composition constrained codes on the stationary memoryless channels have been analyzed extensively, but only for the finite input set case. It is often assumed that all codewords of the code have precisely the same composition. If that is not the case, one usually invokes an expurgation based on the compositions of the codewords and focus on the most populous composition. However, such an expurgation leads to a non-trivial result only when the input set is finite. Bounds derived through the analysis of the most populous composition in such cases can be derived using Augustin information and mean. The following lemma, on the other hand, use the concepts of the Augustin capacity and center together with Augustins averaging in order to avoid assuming a finite input set or a composition that has a fraction of the codewords that is no smaller than a polynomial function of the block length. 
Lemma 9. Let $n \in \mathbb{Z}_{+}, W: X \rightarrow \mathcal{P}(\mathcal{Y})$, $\mathcal{A}$ be a convex subset of $\mathcal{P}(X), \kappa, \phi, \epsilon$ be positive parameters satisfying $\kappa \geq 3$, $\phi<1, \epsilon<1$, and $\gamma$ be

$$
\gamma \triangleq 3 \sqrt[\kappa]{3 n}\left(\left[C_{1 / 2, U_{\mathcal{A}}^{(n)}}+(1-\phi) \ln (1+n)\right] \vee \kappa\right)
$$

If $W_{t}=W$ for all $t \leq n$ and $M, L \in \mathbb{Z}_{+}$are such that $\ln \frac{M}{L}>n \widetilde{C}_{\phi, W, \mathcal{A}}^{\epsilon}+\frac{\gamma}{1-\phi}+\ln \frac{8 e^{3} n^{1.5}}{\epsilon}$, then any $(M, L)$ channel code on $W_{[1, n]}$ satisfying $\Upsilon(\Psi(m)) \in \mathcal{A}$ for all $m \in \mathcal{M}$ satisfies

$$
P_{\mathbf{e}} \geq\left(\frac{\epsilon e^{-2 \gamma}}{8 e^{3} n^{1.5}}\right)^{1 / \phi} e^{-n \widetilde{E}_{s p}^{\epsilon}\left(\frac{1}{n} \ln \frac{M}{L}, W, \mathcal{A}\right)}
$$

The absence of a back-off term for the rate in (44) can be interpreted as an advantage; but this is possible only with a prefactor that makes the bound trivial, i.e. zero, as $\phi$ converges to zero. By changing the analysis slightly it is possible to obtain the following alternative bound:

$$
P_{\mathbf{e}} \geq \frac{\epsilon e^{-2 \gamma}}{8 n^{1.5}} e^{-n \widetilde{E}_{s p}^{\epsilon}(R, W, \mathcal{A})} \quad R=\frac{1}{n} \ln \frac{M}{L}-\frac{1}{n} \ln \frac{8 e^{3} n^{1.5}}{\epsilon e^{-2 \gamma}} .
$$

When considered together with the identity $C_{\alpha, U_{\mathcal{A}(\varrho)}^{(n)}} \leq C_{\alpha, W, n \varrho}$, Lemma 9 and (45) imply Corollary 3 and (46) given in the following.

Corollary 3. Let $n, \ell \in \mathbb{Z}_{+}, W: X \rightarrow \mathcal{P}(\mathcal{Y}), \rho: X \rightarrow \mathbb{R}_{\geq 0}^{\ell}, \varrho \in \Gamma_{\rho}, \kappa, \phi$, $\epsilon$ be positive parameters satisfying $\kappa \geq 3, \phi<1$, $\epsilon<1$, and $\gamma$ be

$$
\gamma \triangleq 3 \sqrt[\kappa]{3 n}\left(\left[C_{1 / 2, W, n \varrho}+(1-\phi) \ln (1+n)\right] \vee \kappa\right)
$$

If $W_{t}=W$ for all $t \leq n$ and $M, L \in \mathbb{Z}_{+}$are such that $\ln \frac{M}{L}>n \widetilde{C}_{\phi, W, \varrho}^{\epsilon}+\frac{\gamma}{1-\phi}+\ln \frac{8 e^{3} n^{1.5}}{\epsilon}$, then any $(M, L)$ channel code on $W_{[1, n]}$ satisfying $\sum_{t=1}^{n} \rho\left(\Psi_{t}(m)\right) \leq n \varrho$ for all $m \in \mathcal{M}$ satisfies

$$
P_{\mathbf{e}} \geq\left(\frac{\epsilon e^{-2 \gamma}}{8 e^{3} n^{1.5}}\right)^{1 / \phi} e^{-n \widetilde{E}_{s p}^{\epsilon}\left(\frac{1}{n} \ln \frac{M}{L}, W, \varrho\right)} .
$$

By changing the the analysis slightly it is possible to obtain the following alternative bound:

$$
P_{\mathbf{e}} \geq \frac{\epsilon e^{-2 \gamma}}{8 n^{1.5}} e^{-n \widetilde{E}_{s p}^{\epsilon}(R, W, \varrho)} \quad R=\frac{1}{n} \ln \frac{M}{L}-\frac{1}{n} \ln \frac{8 e^{3} n^{1.5}}{\epsilon e^{-2 \gamma}} .
$$

Proof of Lemma 9 and (45). The constrained Augustin center $q_{\alpha, W, \mathcal{A}}$ is continuous in $\alpha$ on $(0,1)$ for the total variation topology on $\mathcal{P}(\mathcal{Y})$ by [27, Lemmas $23-(\mathrm{d})$ and 24$]$. Thus $q \cdot, W, \mathcal{A}$ is a transition probability from $((0,1), \mathcal{B}((0,1)))$ to $(\mathcal{Y}, \mathcal{Y})$. For each $t \leq n$, we define the averaged center $q_{\alpha, t}^{\epsilon}$ as the $y$ marginal of the probability measure $u_{\alpha, \epsilon} \circledast q \cdot, W, \mathcal{A}$ where $u_{\alpha, \epsilon}$ is the uniform probability distribution on $(\alpha-\epsilon \alpha, \alpha+\epsilon(1-\alpha))$

$$
q_{\alpha, t}^{\epsilon} \triangleq \frac{1}{\epsilon} \int_{\alpha-\alpha \epsilon}^{\alpha+(1-\alpha) \epsilon} q_{\eta, W, \mathcal{A}} \mathrm{d} \eta \quad \forall t \in\{1, \ldots, n\} .
$$

Let $q_{\alpha, t} \in \mathcal{P}\left(\mathcal{Y}_{t}\right)$ and $q_{\alpha} \in \mathcal{P}\left(\mathcal{Y}_{1}^{n}\right)$ be

$$
\begin{aligned}
q_{\alpha, t} & \triangleq \frac{n}{n+1} q_{\alpha, t}^{\epsilon}+\frac{1}{n+1} q_{1 / 2, U_{\mathcal{A}}^{(n)}} \\
q_{\alpha} & \triangleq \bigotimes_{t=1}^{n} q_{\alpha, t} .
\end{aligned} \quad \forall t \in\{1, \ldots, n\},
$$

Let us denote the probability measure generated by $\Psi(m)$, i.e. $\bigotimes_{t=1}^{n} W\left(\Psi_{t}(m)\right)$, by $w^{m}$ for brevity.

$$
\begin{aligned}
D_{\alpha}\left(w^{m} \| q_{\alpha}\right) & =\sum_{t=1}^{n} D_{\alpha}\left(W\left(\Psi_{t}(m)\right) \| q_{\alpha, t}\right) & & \text { by [37, Thm. 28], } \\
& \leq \sum_{t=1}^{n}\left(\ln \frac{n+1}{n}+D_{\alpha}\left(W\left(\Psi_{t}(m)\right) \| q_{\alpha, t}^{\epsilon}\right)\right) & & \text { by [27, Lemma 1] because } \frac{n}{n+1} q_{\alpha, t}^{\epsilon} \leq q_{\alpha, t}, \\
& \leq 1+\sum_{t=1}^{n} D_{\alpha}\left(W\left(\Psi_{t}(m)\right) \| q_{\alpha, t}^{\epsilon}\right) & & \text { by } \ln \tau \leq \tau-1, \\
& \leq 1+\sum_{t=1}^{n} \frac{1}{\epsilon} \int_{\alpha(1-\epsilon)}^{\alpha+(1-\alpha) \epsilon} D_{\alpha}\left(W\left(\Psi_{t}(m)\right) \| q_{\eta, W, \mathcal{A}}\right) \mathrm{d} \eta & & \text { by the Jensen's inequality and [37, Thm. 12], } \\
& =1+\frac{n}{\epsilon} \int_{\alpha(1-\epsilon)}^{\alpha+(1-\alpha) \epsilon} D_{\alpha}\left(W \| q_{\eta}, W, \mathcal{A} \mid \Upsilon(\Psi(m))\right) \mathrm{d} \eta & & \text { by Definition 3 and the definition of } \Upsilon, \\
& \left.\leq 1+\frac{n}{\epsilon} \int_{\alpha(1-\epsilon)}^{\alpha+(1-\alpha) \epsilon}\left(1 \vee \frac{1-\eta}{\eta} \frac{\alpha}{1-\alpha}\right) D_{\eta}\left(W \| q_{\eta}, W, \mathcal{A} \mid \Upsilon(\Psi(m))\right)\right) & & \text { by [37, Thm. 3 and Proposition 2], } \\
& \leq 1+\frac{n}{\epsilon} \int_{\alpha(1-\epsilon)}^{\alpha+(1-\alpha) \epsilon}\left(1 \vee \frac{1-\eta}{\eta} \frac{\alpha}{1-\alpha}\right) C_{\eta, W, \mathcal{A}} \mathrm{d} \eta & & \text { by [27, Thm. 1] because } \Upsilon(\Psi(m)) \in \mathcal{A}, \\
& =1+n \widetilde{C}_{\alpha, W, \mathcal{A}}^{\epsilon} & & \text { by the definition of } \widetilde{C}_{\alpha, W, \mathcal{A}}^{\epsilon} .
\end{aligned}
$$


Let the probability measure $v_{\alpha}^{m}$ be the order $\alpha$ tilted probability measure between $w^{m}$ and $q_{\alpha}$ defined in (2). Then $v_{\alpha}^{m}$ is of the form $v_{\alpha}^{m}=\bigotimes_{t=1}^{n} W_{\alpha}^{q_{\alpha, t}}\left(\Psi_{t}(m)\right)$ as a result of product structure of $w^{m}$ and $q_{\alpha}$. Let the random variables $\xi_{\alpha, t}^{m}$ and $\xi_{\alpha}^{m}$ be

$$
\begin{aligned}
\xi_{\alpha, t}^{m} & \triangleq \ln \frac{\mathrm{d}\left[W\left(\Psi_{t}(m)\right)\right]_{a c}}{\mathrm{~d} q_{\alpha, t}}-\mathbf{E}_{v_{\alpha}^{m}}\left[\ln \frac{\mathrm{d}\left[W\left(\Psi_{t}(m)\right)\right]_{a c}}{\mathrm{~d} q_{\alpha, t}}\right], \\
\xi_{\alpha}^{m} & \triangleq \sum_{t=1}^{n} \xi_{\alpha, t}^{m},
\end{aligned}
$$

where $\left[W\left(\Psi_{t}(m)\right)\right]_{a c}$ is the component of $W\left(\Psi_{t}(m)\right)$ that is absolutely continuous in $q_{\alpha, t}$. Then for all $\kappa \in \mathbb{R}_{+}$and $\alpha \in(0,1)$

$$
\begin{aligned}
\mathbf{E}_{v_{\alpha}^{m}}\left[\left|\xi_{\alpha, t}^{m}\right|^{\kappa}\right]^{1 / \kappa} & \leq 3^{1 / \kappa} \frac{\left[(1-\alpha) D_{\alpha}\left(W\left(\Psi_{t}(m)\right) \| q_{\alpha, t}\right)\right] \vee \kappa}{\alpha(1-\alpha)} \\
& \leq 3^{1 / \kappa} \frac{\left[(1-\alpha) D_{\alpha}\left(W\left(\Psi_{t}(m)\right) \| q_{1 / 2, U_{\mathcal{A}}^{(n)}}\right)+(1-\alpha) \ln (1+n)\right] \vee \kappa}{\alpha(1-\alpha)} \\
& \leq 3^{1 / \kappa} \frac{\left[D_{1 / 2}\left(W\left(\Psi_{t}(m)\right) \| q_{1 / 2, U_{\mathcal{A}}^{(n)}}\right)+(1-\alpha) \ln (1+n)\right] \vee \kappa}{\alpha(1-\alpha)} \\
& \leq 3^{1 / \kappa} \frac{\left[C_{1 / 2, U_{\mathcal{A}}^{(n)}}+(1-\alpha) \ln (1+n)\right] \vee \kappa}{\alpha(1-\alpha)}
\end{aligned}
$$

by [26, Lemma 17],

by [27, Lemma 1] because $\frac{q_{1 / 2, U_{\mathcal{A}}^{(n)}}}{n+1} \leq q_{\alpha, t}$,

by [37, Thm. 3 and Proposition 2],

by [27, Thm. 1] because $\Psi_{t}(m) \in X_{\mathcal{A}}^{(n)}$.

Then using the definition of $\gamma$ given in (43), we get

$$
\left[\sum_{t=1}^{n} \mathbf{E}_{v_{\alpha}^{m}}\left[\left|\xi_{\alpha, t}^{m}\right|^{\kappa}\right]\right]^{1 / \kappa} \leq \frac{\gamma}{3 \alpha(1-\alpha)} \quad \forall \alpha \in[\phi, 1) .
$$

On the other hand, [37, Thm. 30] implies

$$
D_{1}\left(v_{\alpha}^{m} \| q_{\alpha}\right)=D_{\alpha}\left(w^{m} \| q_{\alpha}\right)-\frac{\alpha}{1-\alpha} D_{1}\left(v_{\alpha}^{m} \| w^{m}\right) \quad \forall m \in \mathcal{M}, \alpha \in(0,1) .
$$

Thus we can bound $D_{1}\left(v_{\alpha}^{m} \| q_{\alpha}\right)$ using the non-negativity of the Rényi divergence, i.e. [37, Thm. 8], and (48)

$$
0 \leq D_{1}\left(v_{\alpha}^{m} \| q_{\alpha}\right) \leq 1+n \widetilde{C}_{\alpha, W, \mathcal{A}}^{\epsilon}
$$

Hence,

$$
\begin{aligned}
& \lim _{\alpha \downarrow \phi} D_{1}\left(v_{\alpha}^{m} \| q_{\alpha}\right)+\frac{\gamma}{1-\alpha}<\ln \frac{M}{L} \frac{\epsilon}{8 e^{2} n^{1.5}}, \\
& \lim _{\alpha \uparrow 1} D_{1}\left(v_{\alpha}^{m} \| q_{\alpha}\right)+\frac{\gamma}{1-\alpha}=\infty .
\end{aligned}
$$

$D_{1}\left(v_{\alpha}^{m} \| q_{\alpha}\right)$ is continuous in $\alpha$ by [26, Lemma 16] because $q_{\alpha}$ is continuous in $\alpha$ for the total variation topology ${ }^{6}$ on $\mathcal{P}(\mathcal{Y})$. Then as a result of the intermediate value theorem [38,4.23], for each $m \in \mathcal{M}$ there exists an $\alpha_{m}$ in $(\phi, 1)$ satisfying

$$
\left.\left(D_{1}\left(v_{\alpha}^{m} \| q_{\alpha}\right)+\frac{\gamma}{1-\alpha}\right)\right|_{\alpha=\alpha_{m}}=\ln \frac{M}{L} \frac{\epsilon}{8 e^{2} n^{1.5}} .
$$

For any $K \in \mathbb{Z}_{+}$, there exists a $1 / K$ long closed subinterval of $(0,1)$ with $\left\lceil\frac{M}{K}\right\rceil$ or more of these $\alpha_{m}$ 's. Let $[\eta, \eta+1 / K]$ be this interval and $\widetilde{q}$ and $\widetilde{q_{t}}$ be

$$
\widetilde{q} \triangleq \bigotimes_{t=1}^{n} \widetilde{q}_{t}
$$$$
\widetilde{q}_{t}=\frac{n}{n+1} q_{\widetilde{\alpha}, t}^{\widetilde{\tau}}+\frac{1}{n+1} q_{1 / 2, U_{\mathcal{A}}^{(n)}},
$$

where $\widetilde{\epsilon}$ and $\widetilde{\alpha}$ are given by

$$
\widetilde{\epsilon}=\frac{1}{K}+\epsilon\left(1-\frac{1}{K}\right) \quad \widetilde{\alpha}=\frac{1-\epsilon}{1-\widetilde{\epsilon}} \eta .
$$

Then for all $\alpha$ in $\left[\eta, \eta+\frac{1}{K}\right]$ using the definition of the averaged center $q_{\alpha, t}^{\epsilon}$ given in (47) we get

$$
q_{\alpha, t} \leq \widetilde{\frac{\epsilon}{\epsilon}} \widetilde{q_{t}} \quad q_{\alpha} \leq\left(\frac{\widetilde{\epsilon}}{\epsilon}\right)^{n} \widetilde{q} .
$$

At least half of the messages with $\alpha_{m}$ 's in $[\eta, \eta+1 / K]$, i.e. at least $\left\lceil\frac{1}{2}\left\lceil\frac{M}{K}\right\rceil\right\rceil$ messages, satisfy $\widetilde{q}\left(\mathcal{E}_{m}\right) \leq 2 \frac{L}{\lceil M / K\rceil}$ as a result of Markov's inequality because $\sum_{m \in \widetilde{\mathcal{M}}} \widetilde{q}\left(\mathcal{E}_{m}\right) \leq L$ by the definition of list decoding, where $\widetilde{\mathcal{M}}=\left\{m: \alpha_{m} \in[\eta, \eta+1 / K]\right\}$. Then at least $\left\lceil\frac{1}{2}\left\lceil\frac{M}{K}\right\rceil\right\rceil$ messages with $\alpha_{m}$ 's in $[\eta, \eta+1 / K]$ satisfy

$$
\begin{aligned}
q_{\alpha_{m}}\left(\mathcal{E}_{m}\right) & \leq \frac{2 L K}{M}\left(1+\frac{1}{K} \frac{1-\epsilon}{\epsilon}\right)^{n} \\
& \leq \frac{2 L K}{M}\left(1+\frac{1}{K \epsilon}\right)^{n} .
\end{aligned}
$$

${ }^{6}$ In particular $\left\|q_{\alpha}-q_{\eta}\right\| \leq \sqrt{8 \ln \frac{\epsilon}{\epsilon-(1-\epsilon)|\alpha-\eta|}}$ because $\left\|q_{\alpha}-q_{\eta}\right\| \leq \sqrt{4 D_{1 / 2}\left(q_{\alpha} \| q_{\eta}\right)}$ by [37, Thm. 31], $D_{1 / 2}\left(q_{\alpha} \| q_{\eta}\right)=n D_{1 / 2}\left(q_{\alpha, t} \| q_{\eta, t}\right)$ by [37, Thm. 28] and the definition of $q_{\alpha}$ 's, $D_{1 / 2}\left(q_{\alpha, t} \| q_{\eta, t}\right) \leq 2 \ln \frac{2}{2-\left\|q_{\alpha, t}-q_{\eta, t}\right\|}$ by [27, (9)], and $\left\|q_{\alpha, t}-q_{\eta, t}\right\| \leq 2 \frac{1-\epsilon}{\epsilon}|\eta-\alpha|$ by the definition of $q_{\alpha}$ 's. 
Note that $\frac{n}{\epsilon}>1$ because we have assumed that $\epsilon<1$ and $n \geq 1$. If we set $K$ to be $K=\left\lfloor\frac{n}{\epsilon}\right\rfloor$ and use the identity $(1+\tau)^{1 / \tau}<e$ together with (51) we get

$$
\begin{aligned}
q_{\alpha_{m}}\left(\mathcal{E}_{m}\right) & \leq \frac{2 L K}{M}\left(1+\frac{1}{K \epsilon}\right) \text { Ke } \frac{n}{K e} \\
& \leq \frac{2 L}{M} \frac{n}{\epsilon} e^{2} \\
& \leq \frac{1}{4 \sqrt{n}} e^{-D_{1}\left(v_{\alpha_{m}}^{m} \| q_{\alpha_{m}}\right)-\frac{\gamma}{1-\alpha_{m}}}
\end{aligned}
$$

Then (49) and Lemma 7 imply

$$
P_{\mathbf{e}}^{m} \geq \frac{1}{4 \sqrt{n}} e^{-D_{1}\left(v_{\alpha_{m}}^{m} \| w^{m}\right)-\frac{\gamma}{\alpha_{m}}}
$$

Using (48), (50), (51), and (52) we get

$$
P_{\mathbf{e}}^{m} \geq \frac{1}{4 \sqrt{n}}\left(\frac{\epsilon}{8 e^{2} n^{1.5}} \frac{M}{L}\right)^{\frac{1-\alpha_{m}}{\alpha_{m}}} e^{-\frac{1-\alpha_{m}}{\alpha_{m}}\left(1+n \widetilde{C}_{\alpha_{m}, W}^{\epsilon}\right)-\frac{2 \gamma}{\alpha_{m}}} .
$$

Hence, for all $m$ satisfying $(51)$ as a result of the definition of $\widetilde{E}_{s p}^{\epsilon}(R, W, \mathcal{A})$ given in (40) we have

$$
P_{\mathbf{e}}^{m} \geq \frac{e^{-2 \gamma / \phi}}{4 \sqrt{n}}\left(\frac{\epsilon}{8 e^{3} n^{1.5}}\right)^{\frac{1-\phi}{\phi}} e^{-n \widetilde{E}_{s p}^{\epsilon}(R, W, \mathcal{A})} \quad R=\frac{1}{n} \ln \frac{M}{L} .
$$

Since there are at least $\left\lceil\frac{1}{2}\left\lceil\frac{M}{K}\right\rceil\right\rceil$ such messages and $\left\lceil\frac{1}{2}\left\lceil\frac{M}{K}\right\rceil\right\rceil \geq \frac{M \epsilon}{2 n}$ by construction, we get the lower bound given in (44).

Note that $\frac{\epsilon e^{-2 \gamma}}{8 e^{3} n^{1.5}}<1$ because $\epsilon \in(0,1), n \geq 1$, and $\gamma \geq 0$. Thus the prefactor in (44) converges to zero as $\phi$ goes to zero. In order avoid this phenomena, one can change the analysis after (53) and introduce an approximation error term to the rate of the averaged SPE term: For all for all $m$ satisfying (51) as a result of the definition of $\widetilde{E}_{s p}^{\epsilon}(R, W, \varrho)$ given in (40) and (53) we have

$$
P_{\mathbf{e}}^{m} \geq \frac{e^{-2 \gamma}}{4 \sqrt{n}} e^{-n \widetilde{E}_{s p}^{\epsilon}(R, W)} \quad R=\frac{1}{n} \ln \frac{M}{L}-\frac{2 \gamma}{n}-\frac{1}{n} \ln \frac{8 e^{3} n^{1.5}}{\epsilon} .
$$

Since there are at least $\left\lceil\frac{1}{2}\left\lceil\frac{M}{K}\right\rceil\right\rceil$ such messages and $\left\lceil\frac{1}{2}\left\lceil\frac{M}{K}\right\rceil\right\rceil \geq \frac{M \epsilon}{2 n}$ by construction, we get the lower bound given in (45).

\subsection{Non-asymptotic Sphere Packing Bounds for the Cost Constrained Codes}

In $\$ 3.3$, we were primarily interested in the stationary memoryless channels with convex composition constraints; the cost constrained memoryless channels, considered in Corollary 3, were merely an afterthought. For the cost constrained memoryless channels it is possible to establish the SPB even for the non-stationary channels using the convex-conjugation techniques, as we will demonstrate in the following. The only drawback of the use the convex-conjugation techniques is that we will be able to establish the SPB for the cost constraints in int $\Gamma_{\rho}$, rather than the cost constraints in $\Gamma_{\rho}$.

Lemma 10. Let $n \in \mathbb{Z}_{+}, W_{[1, n]}: X_{1}^{n} \rightarrow \mathcal{P}\left(\mathcal{Y}_{1}^{n}\right)$ be a length $n$ product channel with an additive cost function $\rho_{[1, n]}: X_{1}^{n} \rightarrow \mathbb{R}_{\geq 0}^{\ell}$

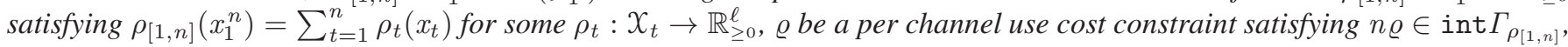
$\kappa, \phi, \epsilon$ be arbitrary positive parameters satisfying $\kappa \geq 3, \phi<1, \epsilon<1$, and $\gamma$ be

$$
\gamma \triangleq 3\left[3 \sum_{t=1}^{n}\left(\left[C_{1 / 2}, W_{t}, n \varrho+(1-\phi) \ln (1+n)\right] \vee \kappa\right)^{\kappa}\right]^{1 / \kappa}
$$

If $M$ and $L$ are integers such that $\ln \frac{M}{L}>\widetilde{C}_{\phi, W_{[1, n]}, n \varrho}^{\epsilon}+\frac{\gamma}{1-\phi}+\ln \frac{8 e^{3} n^{1.5}}{\epsilon}$, then any $(M, L)$ channel code on $W_{[1, n]}$ satisfying $\bigvee_{m=1}^{M} \rho_{[1, n]}(\Psi(m)) \leq n \varrho$ satisfies

$$
P_{\mathbf{e}} \geq\left(\frac{\epsilon e^{-2 \gamma}}{8 e^{3} n^{1.5}}\right)^{1 / \phi} e^{-\widetilde{E}_{s p}^{\epsilon}\left(\ln \frac{M}{L}, W_{[1, n]}, n \varrho\right)}
$$

The bound given in (55) does not have a back-off term for the rate in the averaged SPE but it becomes trivial, i.e. zero, as $\phi$ converges to zero. By changing the analysis slightly it is possible to obtain the following alternative bound:

$$
P_{\mathbf{e}} \geq \frac{\epsilon e^{-2 \gamma}}{8 n^{1.5}} e^{-\widetilde{E}_{s p}^{\epsilon}\left(R, W_{[1, n]}, n \varrho\right)} \quad R=\ln \frac{M}{L}-\ln \frac{8 e^{3} n^{1.5}}{\epsilon e^{-2 \gamma}} .
$$

The bounds in Lemma 10 and (56) are good enough for proving the asymptotic SPB given in Theorem 2 . However, the $\gamma$ term can be improved by a more careful calculation. The resulting expression, however, includes an additional optimization.

Lemma 11. Lemma 10 and (56) are valid as they are if the definition of $\gamma$ given in (54) is replaced by the one given in (57).

$$
\gamma \triangleq \max _{\left\{\varrho_{t}\right\}: \varrho_{t} \geq 0 \forall t, \sum_{t=1}^{n} \varrho_{t} \leq n \varrho} 3\left[3 \sum_{t=1}^{n}\left(\left[C_{1 / 2, W_{t}}^{\lambda}+\lambda \cdot \varrho_{t}+(1-\phi) \ln (1+n)\right] \vee \kappa\right)^{\kappa}\right]^{1 / \kappa}
$$

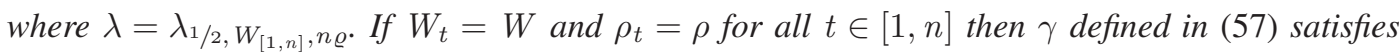

$$
\gamma=\max _{\left\{\varrho_{t}\right\}: \varrho_{t} \geq 0 \forall t, \sum_{t=1}^{n} \varrho_{t} \leq n \varrho} 3\left[3 \sum_{t=1}^{n}\left(\left[C_{1 / 2, W, \varrho}+\lambda \cdot\left(\varrho_{t}-\varrho\right)+(1-\phi) \ln (1+n)\right] \vee \kappa\right)^{\kappa}\right]^{1 / \kappa} .
$$


Proof of Lemma 10 and (56). For each $\alpha \in(0,1)$ there exists a $\lambda_{\alpha} \in \mathbb{R}_{\geq 0}^{\ell}$ satisfying

$$
C_{\alpha, W_{[1, n]}, n \varrho}=C_{\alpha, W_{[1, n]}}^{\lambda_{\alpha}}+\lambda_{\alpha} \cdot \varrho n
$$

by [27, Lemma 29-(c)] because $n \varrho \in \operatorname{int} \Gamma_{\rho_{[1, n]}}$. Hence, $q_{\alpha, W_{[1, n]}, n \varrho}=q_{\alpha, W_{[1, n]}}^{\lambda_{\alpha}}$ by [27, Lemma 31]. Furthermore, $q_{\alpha, W_{[1, n]}}^{\lambda_{\alpha}}$ is of the form $q_{\alpha, W_{[1, n]}}^{\lambda_{\alpha}}=\bigotimes_{t=1}^{n} q_{\alpha, W_{t}}^{\lambda_{\alpha}}$ by [27, Lemma 32]. Then

$$
\begin{aligned}
\left\|q_{\alpha, W_{t}}^{\lambda_{\alpha}}-q_{\phi, W_{t}}^{\lambda_{\phi}}\right\|^{2} & \leq 4 D_{1 / 2}\left(q_{\alpha, W_{t}}^{\lambda_{\alpha}} \| q_{\phi, W_{t}}^{\lambda_{\phi}}\right) \\
& \leq 4 D_{1 / 2}\left(q_{\alpha, W_{[1, n]}}^{\lambda_{\alpha}} \| q_{\phi, W_{[1, n]}}^{\lambda_{\phi}}\right) \\
& =4 D_{1 / 2}\left(q_{\alpha, W_{[1, n]}, n \varrho} \| q_{\phi, W_{[1, n]}, n \varrho}\right) \\
& \leq 8 \ln \frac{2}{2-\left\|q_{\alpha, W_{[1, n]}, \varrho}-q_{\phi, W_{[1, n]}, \varrho}\right\|}
\end{aligned}
$$

by [37, Thm. 31],

by [37, Thms. 8 and 28],

because $q_{\alpha, W_{[1, n]}, n \varrho}=q_{\alpha, W_{[1, n]}}^{\lambda_{\alpha}} \forall \alpha \in(0,1)$,

by $[27,(9)]$.

On the other hand, the constrained Augustin center $q_{\alpha, W_{[1, n]}, n \varrho}$ is continuous in $\alpha$ on $(0,1)$ for the total variation topology on $\mathcal{P}\left(\mathcal{Y}_{1}^{n}\right)$ by [27, Lemmas 23-(d) and 24]. Thus $q_{\alpha, W_{t}}^{\lambda_{\alpha}}$ is a continuous function of $\alpha$ for the total variation topology on $\mathcal{P}\left(\mathcal{Y}_{t}\right)$, as well. Then $q_{\cdot, W_{t}}^{\lambda}$ is a transition probability from $((0,1), \mathcal{B}((0,1)))$ to $\left(y_{t}, \mathcal{Y}_{t}\right)$. We define $q_{\alpha, t}^{\epsilon}$ as the $y_{t}$ marginal of the probability measure $u_{\alpha, \epsilon} \circledast q^{\lambda ., W_{t}} W_{,}$where $u_{\alpha, \epsilon}$ is the uniform probability distribution on $(\alpha-\epsilon \alpha, \alpha+\epsilon(1-\alpha))$ :

$$
q_{\alpha, t}^{\epsilon}=\frac{1}{\epsilon} \int_{\alpha-\alpha \epsilon}^{\alpha+(1-\alpha) \epsilon} q_{\eta, W_{t}}^{\lambda_{\eta}} \mathrm{d} \eta \quad \forall t \in\{1, \ldots, n\} .
$$

Let $q_{\alpha, t} \in \mathcal{P}\left(\mathcal{Y}_{t}\right)$ and $q_{\alpha} \in \mathcal{P}\left(\mathcal{Y}_{1}^{n}\right)$

$$
\begin{aligned}
q_{\alpha, t} & \triangleq \frac{n}{n+1} q_{\alpha, t}^{\epsilon}+\frac{1}{n+1} q_{1 / 2}, W_{t}, n \varrho
\end{aligned} \quad \forall t \in\{1, \ldots, n\},
$$

Let us denote the probability measure generated by $\Psi(m)$, i.e. $\bigotimes_{t=1}^{n} W_{t}\left(\Psi_{t}(m)\right)$, by $w^{m}$ for brevity.

$$
\begin{aligned}
D_{\alpha}\left(w^{m} \| q_{\alpha}\right) & =\sum_{t=1}^{n} D_{\alpha}\left(W_{t}\left(\Psi_{t}(m)\right) \| q_{\alpha, t}\right) & & \text { by [37, Thm. 28], } \\
& \leq \sum_{t=1}^{n}\left(\ln \frac{n+1}{n}+D_{\alpha}\left(W_{t}\left(\Psi_{t}(m)\right) \| q_{\alpha, t}^{\epsilon}\right)\right) & & \text { by [27, Lemma 1] because } \frac{n}{n+1} q_{\alpha, t}^{\epsilon} \leq q_{\alpha, t}, \\
& \leq 1+\sum_{t=1}^{n} D_{\alpha}\left(W_{t}\left(\Psi_{t}(m)\right) \| q_{\alpha, t}^{\epsilon}\right) & & \text { by } \ln \tau \tau-1, \\
& \leq 1+\sum_{t=1}^{n} \frac{1}{\epsilon} \int_{\alpha(1-\epsilon)}^{\alpha+(1-\alpha) \epsilon} D_{\alpha}\left(W_{t}\left(\Psi_{t}(m)\right) \| q_{\eta, W_{t}}^{\lambda_{\eta}}\right) \mathrm{d} \eta & & \text { by the Jensen's inequality and [37, Thm. 12], } \\
& =1+\frac{1}{\epsilon} \int_{\alpha(1-\epsilon)}^{\alpha+(1-\alpha) \epsilon} D_{\alpha}\left(w^{m} \| q_{\eta, W_{[1, n]}}^{\lambda_{\eta}}\right) \mathrm{d} \eta & & \text { by [37, Thm. 28] and [27, Lemma 32], } \\
& \leq 1+\frac{1}{\epsilon} \int_{\alpha(1-\epsilon)}^{\alpha+(1-\alpha) \epsilon}\left(1 \vee \frac{1-\eta}{\eta} \frac{\alpha}{1-\alpha}\right) D_{\eta}\left(w^{m} \| q_{\eta, W_{[1, n]}}^{\lambda_{\eta}}\right) \mathrm{d} \eta & & \text { by [37, Thm. 3 and Proposition 2], } \\
& \leq 1+\frac{1}{\epsilon} \int_{\alpha(1-\epsilon)}^{\alpha+(1-\alpha) \epsilon}\left(1 \vee \frac{1-\eta}{\eta} \frac{\alpha}{1-\alpha}\right)\left(C_{\eta, W}^{\lambda_{\eta}}+\lambda_{\eta} \cdot \varrho n\right) \mathrm{d} \eta & & \text { by [27, Thm. 2] because } \rho_{[1, n]}(\Psi(m)) \leq n \varrho, \\
& =1+\widetilde{C}_{\alpha, W, \varrho}^{\epsilon} \quad & & \text { by (39) and (59). }
\end{aligned}
$$

Let $v_{\alpha}^{m}$ be the order $\alpha$ tilted probability measure between $w^{m}$ and $q_{\alpha}$ defined in (2). Then $v_{\alpha}^{m}$ is a probability measure of the form $v_{\alpha}^{m}=\bigotimes_{t=1}^{n}\left[W_{t}\right]_{\alpha}^{q_{\alpha, t}}\left(\Psi_{t}(m)\right)$ as a result of the product structure of $w^{m}$ and $q_{\alpha}$. Let the random variables $\xi_{\alpha, t}^{m}$ and $\xi_{\alpha}^{m}$ be

$$
\begin{aligned}
\xi_{\alpha, t}^{m} & \triangleq \ln \frac{\mathrm{d}\left[W_{t}\left(\Psi_{t}(m)\right)\right]_{a c}}{\mathrm{~d} q_{\alpha, t}}-\mathbf{E}_{v_{\alpha}^{m}}\left[\ln \frac{\mathrm{d}\left[W_{t}\left(\Psi_{t}(m)\right)\right]_{a c}}{\mathrm{~d} q_{\alpha, t}}\right], \\
\xi_{\alpha}^{m} & \triangleq \sum_{t=1}^{n} \xi_{\alpha, t}^{m},
\end{aligned}
$$

where $\left[W_{t}\left(\Psi_{t}(m)\right)\right]_{a c}$ is the component of $W_{t}\left(\Psi_{t}(m)\right)$ that is absolutely continuous in $q_{\alpha, t}$. Then for all $\kappa \in \mathbb{R}_{+}$and $\alpha \in(0,1)$

$$
\begin{aligned}
\mathbf{E}_{v_{\alpha}^{m}}\left[\left|\xi_{\alpha, t}^{m}\right|^{\kappa}\right]^{1 / \kappa} & \leq 3^{1 / \kappa} \frac{\left[(1-\alpha) D_{\alpha}\left(W_{t}\left(\Psi_{t}(m)\right) \| q_{\alpha, t}\right)\right] \vee \kappa}{\alpha(1-\alpha)} \\
& \leq 3^{1 / \kappa} \frac{\left[(1-\alpha) D_{\alpha}\left(W_{t}\left(\Psi_{t}(m)\right) \| q_{1 / 2, W_{t}, n \varrho}\right)+(1-\alpha) \ln (1+n)\right] \vee \kappa}{\alpha(1-\alpha)} \\
& \leq 3^{1 / \kappa} \frac{\left[D_{1 / 2}\left(W_{t}\left(\Psi_{t}(m)\right) \| q_{1 / 2, W_{t}, n \varrho}\right)+(1-\alpha) \ln (1+n)\right] \vee \kappa}{\alpha(1-\alpha)} \\
& \leq 3^{1 / \kappa} \frac{\left[C_{1 / 2, W_{t}, n \varrho}+(1-\alpha) \ln (1+n)\right] \vee \kappa}{\alpha(1-\alpha)}
\end{aligned}
$$

by [26, Lemma 17],

by [27, Lemma 1] because $\frac{q_{1 / 2, W_{t}, n \varrho}}{n+1} \leq q_{\alpha, t}$, by [37, Thm. 3 and Proposition 2],

by [27, Thm. 1] because $\rho_{t}\left(\Psi_{t}(m)\right) \leq n \varrho$. 
Then using the definition of $\gamma$ given in (54), we get

$$
\left[\sum_{t=1}^{n} \mathbf{E}_{v_{\alpha}^{m}}\left[\left|\xi_{\alpha, t}^{m}\right|^{\kappa}\right]\right]^{1 / \kappa} \leq \frac{\gamma}{3 \alpha(1-\alpha)} \quad \forall \alpha \in[\phi, 1) .
$$

The rest of the proof is analogous to the proof of Lemma 9 and (45) after (49). The differences that might worth mentioning are that bounds given in (60) and (61) are invoked instead of (48) and (49), and $\widetilde{q}_{t}=\frac{n}{n+1} q_{\widetilde{\alpha}, t}^{\widetilde{\epsilon}}+\frac{1}{n+1} q_{1 / 2, W_{t}, \varrho}$ is used instead of $\widetilde{q}_{t}=\frac{n}{n+1} q_{\widetilde{\alpha}, t}^{\widetilde{\epsilon}}+\frac{1}{n+1} q_{1 / 2, U_{\mathcal{A}}^{(n)}}$.

Proof of Lemma 11. The proof is identical to that of Lemma 10 and (56) except for the definition of $q_{\alpha, t}$ and the bound on $\mathbf{E}_{v_{\alpha}^{m}}\left[\left|\xi_{\alpha, t}^{m}\right|^{\kappa}\right]^{1 / \kappa}$. In particular, we set $q_{\alpha, t} \in \mathcal{P}\left(\mathcal{Y}_{t}\right)$ to be $\frac{n}{n+1} q_{\alpha, t}^{\epsilon}+\frac{1}{n+1} q_{1 / 2, W_{t}}^{\lambda_{1 / 2}}$ for all $t \leq n$ and bound $\mathbf{E}_{v_{\alpha}^{m}}\left[\left|\xi_{\alpha, t}^{m}\right|^{\kappa}\right]^{1 / \kappa}$ as follows

$$
\begin{aligned}
\mathbf{E}_{v_{\alpha}^{m}}\left[\left|\xi_{\alpha, t}^{m}\right|^{\kappa}\right]^{1 / \kappa} & \leq 3^{1 / \kappa} \frac{\left[(1-\alpha) D_{\alpha}\left(W_{t}\left(\Psi_{t}(m)\right) \| q_{\alpha, t}\right)\right] \vee \kappa}{\alpha(1-\alpha)} \\
& \leq 3^{1 / \kappa} \frac{\left[(1-\alpha) D_{\alpha}\left(W_{t}\left(\Psi_{t}(m)\right) \| q_{1 / 2}^{\lambda_{1 / 2}}\right)+(1-\alpha) \ln (1+n)\right] \vee \kappa}{\alpha(1-\alpha)} \\
& \leq 3^{1 / \kappa} \frac{\left[D_{1 / 2}\left(W_{t}\left(\Psi_{t}(m)\right) \| q_{1 / 2}^{\lambda_{1 / 2}}\right)+(1-\alpha) \ln (1+n)\right] \vee \kappa}{\alpha(1-\alpha)} \\
& \leq 3^{1 / \kappa} \frac{\left[C_{1 / 2, W_{t}}^{\lambda_{1 / 2}}+\lambda_{1 / 2} \cdot \rho_{t}\left(\Psi_{t}(m)\right)+(1-\alpha) \ln (1+n)\right] \vee \kappa}{\alpha(1-\alpha)}
\end{aligned}
$$

by [26, Lemma 17],

by [27, Lemma 1] because $\frac{q_{1 / 2, W_{t}}^{\lambda_{1 / 2}}}{n+1} \leq q_{\alpha, t}$,

by [37, Thm. 3 and Proposition 2],

by [27, Thm. 2].

Then using the definition of $\gamma$ given in (57), we get

$$
\left[\sum_{t=1}^{n} \mathbf{E}_{v_{\alpha}^{m}}\left[\left|\xi_{\alpha, t}^{m}\right|^{\kappa}\right]\right]^{1 / \kappa} \leq \frac{\gamma}{3 \alpha(1-\alpha)} \quad \forall \alpha \in[\phi, 1) .
$$

The rest of the proof is identical to that of Lemma 10 and (56).

If $W_{t}=W$ and $\rho_{t}=\rho$ for all $t \in[1, n]$, then $C_{\alpha, W_{[1, n]}, n \varrho}=n C_{\alpha, W, \varrho}$ for all $\varrho \in \Gamma_{\rho}$ and $C_{\alpha, W_{[1, n]}}^{\lambda}=n C_{\alpha, W}^{\lambda}$ for all $\lambda \in \mathbb{R}_{\geq 0}^{\ell}$ by [27, Lemmas 28 and 32]. Then (58) follows from $C_{\alpha, W, \varrho}=C_{\alpha, W}^{\lambda_{\alpha, W, \varrho}}+\lambda_{\alpha, W, \varrho} \cdot \varrho$, established in [27, Lemma 29-(c)].

\section{EXAMPLES}

As a result of $\$ 2.4$ and $\S 3$, we can conclude that the SPE governs the exponential decay rate of the error probability of channel codes with list decoding on memoryless channels under rather mild hypotheses. The calculation of the SPE itself, however, is a separate issue that is essential from a practical standpoint. In this section, we derive the SPE for various Gaussian and Poisson channels and demonstrate that it is possible to obtain parametric forms for these channels similar to the one given in Lemma 2 for $E_{s p}(R, W, p)$. We believe these parametric forms are more straightforward and intuitive than commonly used equivalent parametric forms that were previously derived.

\subsection{Gaussian Channels}

We denote the probability density function of the zero mean Gaussian random variable with variance $\sigma^{2}$ by $\varphi_{\sigma^{2}}$, i.e.

$$
\varphi_{\sigma^{2}}(z) \triangleq \frac{1}{\sqrt{2 \pi} \sigma} e^{-\frac{z^{2}}{2 \sigma^{2}}} \quad \forall z \in \mathbb{R}
$$

With a slight abuse of notation, we denote the corresponding probability measure on $\mathcal{B}(\mathbb{R})$ by $\varphi_{\sigma^{2}}$, as well.

Example 1 (The Scalar Gaussian Channel). Let $W$ be the scalar Gaussian channel with noise variance $\sigma^{2}$ and the associated cost function $\rho$ be the quadratic one:

$$
\begin{aligned}
W(\mathcal{E} \mid x) & =\int_{\mathcal{E}} \varphi_{\sigma^{2}}(y-x) \mathrm{d} y & & \forall \mathcal{E} \in \mathcal{B}(\mathbb{R}), \\
\rho(x) & =x^{2} & & \forall x \in \mathbb{R} .
\end{aligned}
$$

The cost constrained Augustin capacity and center of this channel are determined in [27, Example 4]:

$$
\begin{aligned}
C_{\alpha, W, \varrho} & =\left\{\begin{array}{ll}
\frac{\alpha \varrho}{2\left(\alpha \theta_{\alpha, \sigma, \varrho}+(1-\alpha) \sigma^{2}\right)}+\frac{1}{\alpha-1} \ln \frac{\left(\theta_{\alpha, \sigma, \varrho}\right)^{\alpha / 2} \sigma^{(1-\alpha)}}{\sqrt{\alpha \theta_{\alpha, \sigma, \varrho}+(1-\alpha) \sigma^{2}}} & \alpha \in \mathbb{R}+\backslash\{1\}, \\
\frac{1}{2} \ln \left(1+\frac{\varrho}{\sigma^{2}}\right) & \alpha=1
\end{array},\right. \\
q_{\alpha, W, \varrho} & =\varphi_{\theta_{\alpha, \sigma, \varrho}}, \\
\theta_{\alpha, \sigma, \varrho} & \triangleq \sigma^{2}+\frac{\varrho}{2}-\frac{\sigma^{2}}{2 \alpha}+\sqrt{\left(\frac{\varrho}{2}-\frac{\sigma^{2}}{2 \alpha}\right)^{2}+\varrho \sigma^{2}} .
\end{aligned}
$$


It is worth mentioning that $C_{\alpha, W, \varrho}=I_{\alpha}\left(\varphi_{\varrho} ; W\right)$ and $q_{\alpha, W, \varrho}=q_{\alpha, \varphi_{\varrho}}$ for all positive orders $\alpha$, i.e. zero mean Gaussian distribution with variance $\varrho$ is the optimal input distribution for all orders. Thus $E_{s p}(R, W, \varrho)=E_{s p}\left(R, W, \varphi_{\varrho}\right)$.

The SPE of the scalar Gaussian channel can be characterized using Lemma 4. To see how, first note that for any $\theta>0$ and the corresponding the Gaussian probability measure $\varphi_{\theta}$, the order $\alpha$ tilted channel $W_{\alpha}^{\varphi_{\theta}}$, defined in (3), is given by

$$
W_{\alpha}^{\varphi_{\theta}}(\mathcal{E} \mid x)=\int_{\mathcal{E}} \varphi \frac{\sigma^{2} \theta}{\alpha \theta+(1-\alpha) \sigma^{2}}\left(y-\frac{\alpha \theta}{\alpha \theta+(1-\alpha) \sigma^{2}} x\right) \mathrm{d} y \quad \forall \mathcal{E} \in \mathcal{B}(\mathbb{R}) .
$$

Since $\theta_{\alpha, \sigma, \varrho}$ is a root of the equality $\theta^{2}-\theta\left[\varrho+\left(2-\frac{1}{\alpha}\right) \sigma^{2}\right]+\left(1-\frac{1}{\alpha}\right) \sigma^{4}=0$ for $\theta$ by [27, (132) and (133)], one can confirm using $[27,(131)]$ by substitution that

$$
\begin{aligned}
D_{1}\left(W_{\alpha}^{\varphi_{\theta_{\alpha, \sigma, \varrho}}} \| \varphi_{\theta_{\alpha, \sigma, \varrho}} \mid p\right) & =\frac{\alpha^{2} \theta_{\alpha, \sigma, \varrho}}{2\left(\alpha \theta_{\alpha, \sigma, \varrho}+(1-\alpha) \sigma^{2}\right)^{2}}\left(\mathbf{E}_{p}[\rho]-\varrho\right)+\frac{1}{2} \ln \frac{\alpha \theta_{\alpha, \sigma, \varrho}+(1-\alpha) \sigma^{2}}{\sigma^{2}} \\
D_{1}\left(W_{\alpha}^{\varphi_{\theta_{\alpha, \sigma, \varrho}}} \| W \mid p\right) & =\frac{(1-\alpha)^{2} \sigma^{2}}{2\left(\alpha \theta_{\alpha, \sigma, \varrho}+(1-\alpha) \sigma^{2}\right)^{2}}\left(\mathbf{E}_{p}[\rho]-\varrho\right)+\frac{(1-\alpha) \varrho}{2\left(\alpha \theta_{\alpha, \sigma, \varrho}+(1-\alpha) \sigma^{2}\right)}+\frac{1}{2} \ln \frac{\alpha \theta_{\alpha, \sigma, \varrho}+(1-\alpha) \sigma^{2}}{\theta_{\alpha, \sigma, \varrho}}
\end{aligned}
$$

Thus for each $\alpha^{*} \in(0,1), V_{p}=W_{\alpha^{*}}^{\varphi_{\theta} \alpha^{*}, \sigma, \varrho}$ satisfies the hypotheses of Lemma 4 given in (21) and (22) for $R=\frac{1}{2} \ln \frac{\alpha^{*} \theta_{\alpha, \sigma, \varrho}+\left(1-\alpha^{*}\right) \sigma^{2}}{\sigma^{2}}$ as a result of (62) and the constraint $\mathbf{E}_{p}[\rho] \leq \varrho$. Furthermore, $f(\alpha) \triangleq \frac{1}{2} \ln \frac{\alpha \theta_{\alpha, \sigma, \varrho}+(1-\alpha) \sigma^{2}}{\sigma^{2}}$ is a continuous and increasing function of $\alpha$ satisfying $\lim _{\alpha \downarrow 0} f(\alpha)=0$ and $f(1)=C_{1, W, \varrho}$. Thus the SPE can be written in the following parametric form in terms of $\alpha \in[0,1]$ for all rates in $\left[0, C_{1, W, \varrho}\right]$ :

$$
\begin{aligned}
R & =\frac{1}{2} \ln \frac{\alpha \theta_{\alpha, \sigma, \varrho}+(1-\alpha) \sigma^{2}}{\sigma^{2}}, \\
E_{s p}(R, W, \varrho) & =\frac{(1-\alpha) \varrho}{2\left(\alpha \theta_{\alpha, \sigma, \varrho}+(1-\alpha) \sigma^{2}\right)}+\frac{1}{2} \ln \frac{\alpha \theta_{\alpha, \sigma, \varrho}+(1-\alpha) \sigma^{2}}{\theta_{\alpha, \sigma, \varrho}} .
\end{aligned}
$$

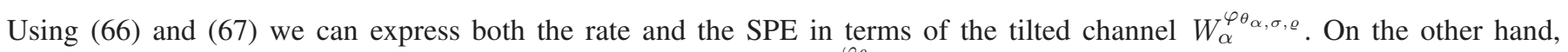
$\varphi_{\theta_{\alpha, \sigma, \varrho}}$ is the output distribution for the input distribution $\varphi_{\varrho}$ on $W_{\alpha}^{\varphi_{\theta_{\alpha}, \sigma, \varrho}}$ because $\varphi_{\theta_{\alpha, \sigma, \varrho}}$ is the Augustin mean $q_{\alpha, \varphi_{\varrho}}$ for the input distribution $\varphi_{\varrho}$ satisfying the fixed point property $\mathrm{T}_{\alpha, \varphi_{\varrho}}\left(q_{\alpha, \varphi_{\varrho}}\right)=q_{\alpha, \varphi_{\varrho}}$, as well. Thus we can rewrite (68) and (69), using (66) and (67), as follows:

$$
\begin{gathered}
R=I_{1}\left(\varphi_{\varrho} ; W_{\alpha}^{\varphi_{\theta_{\alpha}, \sigma, \varrho}}\right), \\
E_{s p}(R, W, \varrho)=D_{1}\left(W_{\alpha}^{\varphi_{\theta_{\alpha, \sigma, \varrho}}} \| W \mid \varphi_{\varrho}\right) .
\end{gathered}
$$

To obtain an expression for the SPE that does not depend on $\theta_{\alpha, \sigma, \varrho}$ explicitly, we first note that (64) and (68) imply

$$
\alpha=\frac{e^{2 R}-1}{2}\left(\sqrt{1+\frac{4 \sigma^{2}}{\varrho} \frac{e^{2 R}}{e^{2 R}-1}}-1\right) .
$$

On the other hand, $\varphi_{\theta_{\alpha, \sigma, \varrho}}$ is the output distribution for the input distribution $\varphi_{\varrho}$ on channel $W_{\alpha}^{\varphi_{\theta_{\alpha}, \sigma, \varrho}}$. Thus (65) implies

$$
\frac{\sigma^{2} \theta_{\alpha, \sigma, \varrho}}{\alpha \theta_{\alpha, \sigma, \varrho}+(1-\alpha) \sigma^{2}}+\left(\frac{\alpha \theta_{\alpha, \sigma, \varrho}}{\alpha \theta_{\alpha, \sigma, \varrho}+(1-\alpha) \sigma^{2}}\right)^{2} \varrho=\theta_{\alpha, \sigma, \varrho}
$$

Thus

$$
\begin{aligned}
\frac{\sigma^{2}}{\theta_{\alpha, \sigma, \varrho}} & =1-\frac{\alpha \varrho}{\alpha \theta_{\alpha, \sigma, \varrho}+(1-\alpha) \sigma^{2}} \\
& =1-\frac{\varrho \alpha}{\sigma^{2} e^{2 R}},
\end{aligned}
$$

where (73) follows from (68).

Using first (68) and (73) in (69), and then invoking (72) we get the following expression for SPE:

$$
\begin{aligned}
E_{s p}(R, W, \varrho) & =\frac{1}{2} \frac{(1-\alpha) \varrho}{\sigma^{2} e^{2 R}}+R+\frac{1}{2} \ln \left(1-\frac{\alpha \varrho}{\sigma^{2} e^{2 R}}\right) \\
& =\frac{\varrho}{4 \sigma^{2}}\left[1+\frac{1}{e^{2 R}}-\left(1-\frac{1}{e^{2 R}}\right) \sqrt{1+\frac{4 \sigma^{2}}{\varrho} \frac{e^{2 R}}{e^{2 R}-1}}\right]+\frac{1}{2} \ln \left[e^{2 R}-\frac{\varrho}{\sigma^{2}}\left(\frac{e^{2 R}-1}{2}\right)\left(\sqrt{\left.\left.1+\frac{4 \sigma^{2}}{\varrho} \frac{e^{2 R}}{e^{2 R}-1}-1\right)\right] .} .\right.\right.
\end{aligned}
$$

The expression given in (74) for the SPE is equivalent to [8, (7.4.33)].

The parametric characterization given in (68) and (69) can be obtained by a more direct approach using the differentiability of $\theta_{\alpha, \sigma, \varrho}$ and $C_{\alpha, W, \varrho}$ in $\alpha$, as well. In particular, since $\theta_{\alpha, \sigma, \varrho}$ is a root of the equality $\theta^{2}-\theta\left[\varrho+\left(2-\frac{1}{\alpha}\right) \sigma^{2}\right]+\left(1-\frac{1}{\alpha}\right) \sigma^{4}=0$ for $\theta$, we get the following closed form expression for the derivative of the Augustin capacity with respect to the order:

$$
\begin{aligned}
\frac{\partial}{\partial \alpha} C_{\alpha, W, \varrho} & =\frac{1}{2(1-\alpha)^{2}}\left[\frac{(1-\alpha) \varrho}{\alpha \theta_{\alpha, \sigma, \varrho}+(1-\alpha) \sigma^{2}}+\ln \frac{\alpha \theta_{\alpha, \sigma, \varrho}+(1-\alpha) \sigma^{2}}{\theta_{\alpha, \sigma, \varrho}}\right]+\frac{\theta_{\alpha, \sigma, \varrho}^{2}-\theta_{\alpha, \sigma, \varrho}\left[\varrho+\left(2-\frac{1}{\alpha}\right) \sigma^{2}\right]+\left(1-\frac{1}{\alpha}\right) \sigma^{4}}{2\left(\alpha \theta_{\alpha, \sigma, \varrho}+(1-\alpha) \sigma^{2}\right)^{2}}\left[\frac{\alpha}{1-\alpha}+\frac{\alpha^{2}}{\theta_{\alpha, \sigma, \varrho}} \frac{\partial}{\partial \alpha} \theta_{\alpha, \sigma, \varrho}\right] \\
& =\frac{1}{2(1-\alpha)^{2}}\left[\frac{(1-\alpha) \varrho}{\alpha \theta_{\alpha, \sigma, \varrho}+(1-\alpha) \sigma^{2}}+\ln \frac{\alpha \theta_{\alpha, \sigma, \varrho}+(1-\alpha) \sigma^{2}}{\theta_{\alpha, \sigma, \varrho}}\right] .
\end{aligned}
$$


Using first (75), and then (62) we get

$$
\begin{aligned}
\frac{\mathrm{d}}{\mathrm{d} \alpha} \frac{1-\alpha}{\alpha}\left(C_{\alpha, W, \varrho}-R\right) & =\frac{1-\alpha}{\alpha}\left(\frac{1}{2(1-\alpha)^{2}}\left[\frac{(1-\alpha) \varrho}{\alpha \theta_{\alpha, \sigma, \varrho}+(1-\alpha) \sigma^{2}}+\ln \frac{\alpha \theta_{\alpha, \sigma, \varrho}+(1-\alpha) \sigma^{2}}{\theta_{\alpha, \sigma, \varrho}}\right]\right)-\frac{1}{\alpha^{2}}\left(C_{\alpha, W, \varrho}-R\right) \\
& =\frac{1}{\alpha^{2}}\left(R-\frac{1}{2} \ln \frac{\alpha \theta_{\alpha, \sigma, \varrho}+(1-\alpha) \sigma^{2}}{\sigma^{2}}\right) .
\end{aligned}
$$

Then the derivative test implies the parametric form given in (68) and (69) as a result of (62).

Example 2 (The Parallel Gaussian Channels). Let $W_{[1, n]}$ be the product of scalar Gaussian channels with noise variance $\sigma_{\imath}^{2}$ for $\imath \in\{1, \ldots, n\}$ and the cost function $\rho_{[1, n]}$ be the additive quadratic one, i.e.

$$
\begin{aligned}
W_{[1, n]}\left(\mathcal{E} \mid x_{1}^{n}\right) & =\int_{\mathcal{E}}\left[\prod_{\imath=1}^{n} \varphi_{\sigma_{\imath}^{2}}\left(y_{\imath}-x_{\imath}\right)\right] \mathrm{d} y_{1}^{n} & & \forall \mathcal{E} \in \mathcal{B}\left(\mathbb{R}^{n}\right), \\
\rho_{[1, n]}\left(x_{1}^{n}\right) & =\sum_{\imath=1}^{n} x_{\imath}^{2} & & \forall x_{1}^{n} \in \mathbb{R}^{n} .
\end{aligned}
$$

The constrained Augustin capacity and center of $W_{[1, n]}$ were determined in [27, Example 5]:

$$
\begin{aligned}
C_{\alpha, W_{[1, n]}, \varrho} & =\sum_{\imath=1}^{n} C_{\alpha, W_{\imath}, \varrho_{\alpha, \imath}}, \\
q_{\alpha, W_{[1, n]}, \varrho} & =\bigotimes_{\imath=1}^{n} \varphi_{\theta_{\alpha, \sigma_{\imath}, \varrho_{\alpha, \imath}},} \\
\varrho_{\alpha, \imath} & =\frac{\left|\alpha-2 \sigma_{\imath}^{2} \lambda_{\alpha}\right|^{+}}{2 \lambda_{\alpha}\left(\alpha+2(\alpha-1) \sigma_{\imath}^{2} \lambda_{\alpha}\right)},
\end{aligned}
$$

where $\theta_{\alpha, \sigma, \varrho}$ is defined in (64) and $\lambda_{\alpha}$ is determined by $\sum_{\imath} \varrho_{\alpha, \imath}=\varrho$ uniquely. ${ }^{7}$ Furthermore, $\theta_{\alpha, \sigma_{\imath}, \varrho_{\alpha, \imath}}$ can be expressed in terms of $\sigma_{\imath}$ and $\lambda_{\alpha}$ without explicitly referring to $\varrho_{\alpha, \imath}$ as follows:

$$
\theta_{\alpha, \sigma_{\imath}, \varrho_{\alpha, \imath}}=\sigma_{\imath}^{2}+\left|\frac{1}{2 \lambda_{\alpha}}-\frac{\sigma_{\imath}^{2}}{\alpha}\right|^{+} .
$$

On the other hand $\frac{\mathrm{d}}{\mathrm{d} \varrho_{\imath}} C_{\alpha, W_{\imath}, \varrho_{\imath}} \varrho_{\varrho_{\imath}=\varrho_{\alpha, \imath}}=\lambda_{\alpha}$ for all $\imath$ 's with a positive $\varrho_{\alpha, \imath}$ and $\left.\frac{\mathrm{d}}{\mathrm{d} \varrho_{\imath}} C_{\alpha, W_{\imath}, \varrho_{\imath}}\right|_{\varrho_{\imath}}=\varrho_{\alpha, \imath} \leq \lambda_{\alpha}$ for all $\imath$ 's. Then using the chain rule of derivatives together with (75) and (62) we get

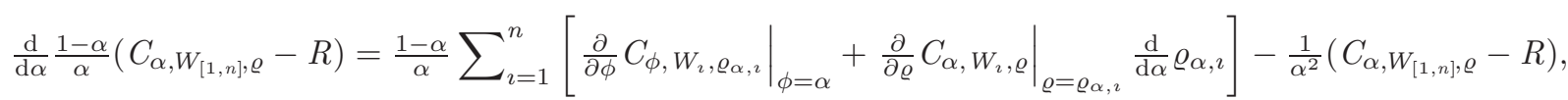

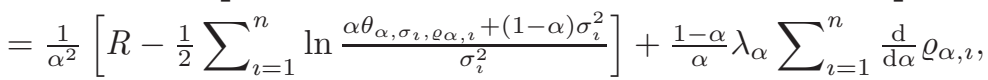

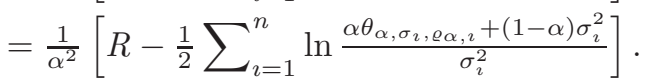

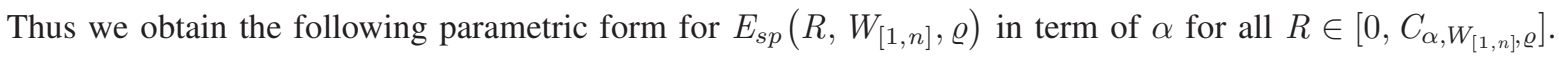

$$
\begin{gathered}
R=\frac{1}{2} \sum_{\imath=1}^{n} \ln \frac{\alpha \theta_{\alpha, \sigma_{\imath}, \varrho_{\alpha, \imath}+(1-\alpha) \sigma_{\imath}^{2}}}{\sigma_{\imath}^{2}}, \\
E_{s p}\left(R, W_{[1, n]}, \varrho\right)=\frac{1}{2} \sum_{\imath=1}^{n}\left[\frac{(1-\alpha) \varrho_{\alpha, \imath}}{\alpha \theta_{\alpha, \sigma_{\imath}, \varrho_{\alpha, \imath}+(1-\alpha) \sigma_{\imath}^{2}}}+\ln \frac{\alpha \theta_{\alpha, \sigma_{\imath}, \varrho_{\alpha, \imath}}+(1-\alpha) \sigma_{\imath}^{2}}{\theta_{\alpha, \sigma_{\imath}, \varrho_{\alpha, \imath}}}\right] .
\end{gathered}
$$

Thus $E_{s p}\left(R, W_{[1, n]}, \varrho\right)=D_{1}\left(V_{\alpha} \| W_{[1, n]} \mid \Phi_{\alpha}\right)$ for $R=I_{1}\left(\Phi_{\alpha} ; V_{\alpha}\right)$ where the input distribution $\Phi_{\alpha}$ is a zero mean the Gaussian distribution with the diagonal covariance matrix whose eigenvalues are $\varrho_{\alpha, 1}, \ldots, \varrho_{\alpha, n}$ and $V_{\alpha}$ is the order $\alpha$ tilted channel between $W_{[1, n]}$ and $q_{\alpha, \Phi_{\alpha}}$.

If $\sigma_{\imath}^{2} \geq \frac{\alpha}{2 \lambda_{\alpha}}$ for an $\imath$, then $\varrho_{\alpha, \imath}=0$ and the corresponding terms in the sums given in (80) and (81) are zero. In [19], Ebert provided an alternative parametric form for the SPE relying on this observation. To obtain Ebert's characterization first note that

$$
\alpha \theta_{\alpha, \sigma_{\imath}, \varrho_{\alpha, \imath}}+(1-\alpha) \sigma_{\imath}^{2}=\frac{\alpha}{2 \lambda_{\alpha}} \vee \sigma_{\imath}^{2} \quad \forall \imath \in\{1, \ldots, n\}
$$

by (79). Thus (79), (80), (81) and the constraint $\sum_{\imath} \varrho_{\alpha, \imath}=\varrho$ imply the following parametric form in terms of $N=\frac{\alpha}{2 \lambda_{\alpha}}$, which is equivalent to Ebert's characterization [8, (7.5.28), (7.5.32), (7.5.34)], [18, p. 294], [19, (20)]

$$
\begin{aligned}
R & =\frac{1}{2} \sum_{\imath: \sigma_{\imath}^{2} \leq N} \ln \frac{N}{\sigma_{\imath}^{2}}, \\
\varrho & =\frac{1}{\alpha} \sum_{\imath=1}^{n} \frac{\left|N-\sigma_{\imath}^{2}\right|^{+}}{1+(\alpha-1) \frac{\sigma_{2}^{2}}{N}}, \\
E_{s p}\left(R, W_{[1, n]}, \varrho\right) & =\frac{(1-\alpha) \varrho}{2 N}+\frac{1}{2} \sum_{\imath: \sigma_{\imath}^{2} \leq N} \ln \frac{\alpha N}{N-(1-\alpha) \sigma_{\imath}^{2}} .
\end{aligned}
$$

Note that one does not need to determine $\lambda_{\alpha}$ or invoke $N=\frac{\alpha}{2 \lambda_{\alpha}}$ in the above expressions. One can first determine $N$ using (82), and then determine $\alpha$ using (83), in order to determine $E_{s p}\left(R, W_{[1, n]}, \varrho\right)$, as noted by Ebert in [18] and [19].

${ }^{7}$ The constraint $\sum_{\imath} \varrho_{\alpha, \imath}=\varrho$ determines $\lambda_{\alpha}$ uniquely because the expression on the right hand side of (78) is a nonincreasing function of $\lambda_{\alpha}$. 


\subsection{Poisson Channels}

Let $T \in \mathbb{R}_{+}$and $a, b \in \mathbb{R}_{\geq 0}$ such that $a \leq b$. Then for the Poisson channel $\Lambda: \mathcal{F} \rightarrow \mathcal{P}(\mathcal{Y})$, the input set $\mathcal{F}$ is the set of all measurable functions of the form $f:(0, T] \rightarrow[a, b]$, the output set $y$ is the set of all nondecreasing, right-continuous, integer valued functions on $(0, T]$, the $\sigma$-algebra of the output events $\mathcal{Y}$ is the Borel $\sigma$-algebra for the topology generated by the Skorokhod metric on $y$, and $\Lambda(f)$ is the Poisson point process with deterministic intensity function $f$ for all $f \in \mathcal{F}$. With a slight abuse of notation we denote the Poisson process with constant intensity $\gamma$ by $\Lambda(\gamma)$. The cost function $\rho: \mathcal{F} \rightarrow \mathbb{R} \geq 0$ is

$$
\rho(f) \triangleq \frac{1}{T} \int_{0}^{T} f(t) \mathrm{d} t .
$$

In $[28, \S \mathrm{V}-\mathrm{C}]$, the (unconstrained) Rényi capacities and centers of various Poisson channels are determined. These expressions are equal to the corresponding Augustin capacities and centers because $C_{\alpha, W}^{0}=C_{\alpha, W}^{g 0}$ for any $W$ and $q_{\alpha, W}^{0}=q_{\alpha, W}^{g 0}$ for any $W$ with finite $C_{\alpha, W}^{0}$ by [27, Thms. 2 and 3].

Example 3 (The Poisson Channels With Given Average Intensity). [28, Example 9] considers $\Lambda^{\varrho}: \mathcal{F}^{\varrho} \rightarrow \mathcal{P}(\mathcal{Y})$ where $\mathcal{F}^{\varrho}=\{f \in \mathcal{F}: \rho(f)=\varrho\}$ and $\Lambda^{\varrho}(f)=\Lambda(f)$ for all $f \in \mathcal{F}$. The Rényi capacity and center of $\Lambda^{\varrho}$ - hence the Augustin capacity and center of $\Lambda^{\varrho}$ - are given in $[28,(73)$ and (74)] to be

$$
\begin{aligned}
C_{\alpha, \Lambda \varrho} & =\left\{\begin{array}{ll}
\frac{\alpha}{\alpha-1}\left(\zeta_{\alpha, \varrho}-\varrho\right) T & \alpha \neq 1 \\
\left(\frac{\varrho-a}{b-a} b \ln \frac{b}{\varrho}+\frac{b-\varrho}{b-a} a \ln \frac{a}{\varrho}\right) T & \alpha=1
\end{array},\right. \\
q_{\alpha, \Lambda \varrho} & =\Lambda\left(\zeta_{\alpha, \varrho}\right), \\
\zeta_{\alpha, \varrho} & \triangleq\left(\frac{\varrho-a}{b-a} b^{\alpha}+\frac{b-\varrho}{b-a} a^{\alpha}\right)^{1 / \alpha} .
\end{aligned}
$$

Since the expression for $C_{\alpha, \Lambda}$ is differentiable in $\alpha$; we obtain the following parametric expression for the SPE using the derivative test

$$
\begin{aligned}
R & =\left(\frac{\varrho-a}{b-a} b^{\alpha} \zeta_{\alpha, \varrho}^{1-\alpha} \ln \frac{b^{\alpha} \zeta_{\alpha, \varrho}^{1-\alpha}}{\zeta_{\alpha, \varrho}}+\frac{b-\varrho}{b-a} a^{\alpha} \zeta_{\alpha, \varrho}^{1-\alpha} \ln \frac{a^{\alpha} \zeta_{\alpha, \varrho}^{1-\alpha}}{\zeta_{\alpha, \varrho}}\right) T \\
E_{s p}\left(R, \Lambda^{\varrho}\right) & =\left(\varrho-\zeta_{\alpha, \varrho}+\frac{\varrho-a}{b-a} b^{\alpha} \zeta_{\alpha, \varrho}^{1-\alpha} \ln \frac{b^{\alpha} \zeta_{\alpha, \varrho}^{1-\alpha}}{b}+\frac{b-\varrho}{b-a} a^{\alpha} \zeta_{\alpha, \varrho}^{1-\alpha} \ln \frac{a^{\alpha} \zeta_{\alpha, \varrho}^{1-\alpha}}{a}\right) T .
\end{aligned}
$$

There is an alternative parametric characterization, which is considerably easier to remember in terms of the tilted channels. In order to derive that expression, first note that $[28,(66),(68)]$ and the definition of the tilted channel given in (3) imply that

$$
\Lambda_{\alpha}^{\Lambda(g)}(f)=\Lambda\left(f^{\alpha} g^{(1-\alpha)}\right)
$$

Then using [28, (68)], (86), (87), (88), (89), we get

$$
\begin{aligned}
R & =D_{1}\left(\Lambda_{\alpha}^{q_{\alpha}, \Lambda^{\varrho}}\left(f_{o p t}\right) \| q_{\alpha, \Lambda^{\varrho}}\right), \\
E_{s p}\left(R, \Lambda^{\varrho}\right) & =D_{1}\left(\Lambda_{\alpha}^{q_{\alpha}, \Lambda^{\varrho}}\left(f_{o p t}\right) \| \Lambda\left(f_{o p t}\right)\right),
\end{aligned}
$$

where $f_{o p t}$ is any $\{a, b\}$ valued function in $\mathcal{F}^{\varrho}$, i.e. any function $f_{o p t}:(0, T] \rightarrow\{a, b\}$ satisfying $\rho\left(f_{\text {opt }}\right)=\varrho$.

Example 4 (The Poisson Channels With Constrained Average Intensity). Let us first confirm that the constrained Augustin capacity $C_{\alpha, \Lambda, \varrho}$ and the constrained Augustin center $q_{\alpha, \Lambda, \varrho}$ are given by ${ }^{8}$

$$
\begin{aligned}
C_{\alpha, \Lambda, \varrho} & =C_{\alpha, \Lambda \varrho \wedge \varrho_{\alpha}}, \\
q_{\alpha, \Lambda, \varrho} & =q_{\alpha, \Lambda \varrho \wedge \varrho_{\alpha}},
\end{aligned}
$$

where $C_{\alpha, \Lambda} \varrho$ and $q_{\alpha, \Lambda}$ are determined by (85), (86), and (87), $\varrho_{\alpha}$ is a decreasing function of the order $\alpha$ defined as

$$
\varrho_{\alpha} \triangleq\left\{\begin{array}{ll}
\alpha^{\frac{\alpha}{1-\alpha}}\left(\frac{b-a}{b^{\alpha}-a^{\alpha}}\right)^{\frac{1}{1-\alpha}}+\frac{a b^{\alpha}-b a^{\alpha}}{b^{\alpha}-a^{\alpha}} & \alpha \neq 1 \\
e^{-1} b^{\frac{b}{b-a}} a^{-\frac{a}{b-a}} & \alpha=1
\end{array} .\right.
$$

To establish (93) and (94), first note that $C_{\alpha, \Lambda \varrho \wedge \varrho_{\alpha}} \leq C_{\alpha, \Lambda, \varrho}$ because $C_{\alpha, \Lambda \varrho \wedge \varrho_{\alpha}}=C_{\alpha, \Lambda, \mathcal{P}\left(\mathcal{F} \varrho \wedge \varrho_{\alpha}\right)}$ and $\mathcal{P}\left(\mathcal{F} \varrho \wedge \varrho_{\alpha}\right) \subset \mathcal{A}(\varrho)$, where $\mathcal{A}(\varrho)=\left\{p \in \mathcal{P}(\mathcal{F}): \mathbf{E}_{p}[\rho(f)] \leq \varrho\right\}$. On the other hand, invoking first [28, (82)], and then [28, (76)], we get

$$
\begin{aligned}
D_{\alpha}\left(\Lambda(f) \| \Lambda\left(\zeta_{\alpha, \varrho \wedge \varrho_{\alpha}}\right)\right) & \leq \frac{b-\rho(f)}{b-a} D_{\alpha}\left(\Lambda(a) \| \Lambda\left(\zeta_{\alpha, \varrho \wedge \varrho_{\alpha}}\right)\right)+\frac{\rho(f)-a}{b-a} D_{\alpha}\left(\Lambda(b) \| \Lambda\left(\zeta_{\alpha, \varrho \wedge \varrho_{\alpha}}\right)\right) \\
& =C_{\alpha, \Lambda \varrho \wedge \varrho_{\alpha}}+\frac{\varrho \wedge \varrho_{\alpha}-\rho(f)}{b-a}\left[D_{\alpha}\left(\Lambda(a) \| \Lambda\left(\zeta_{\alpha, \varrho \wedge \varrho_{\alpha}}\right)\right)-D_{\alpha}\left(\Lambda(b) \| \Lambda\left(\zeta_{\alpha, \varrho \wedge \varrho_{\alpha}}\right)\right)\right] \quad \forall f \in \mathcal{F} .
\end{aligned}
$$

Since $D_{\alpha}\left(\Lambda(a) \| \Lambda\left(\zeta_{\alpha, \varrho_{\alpha}}\right)\right)=D_{\alpha}\left(\Lambda(b) \| \Lambda\left(\zeta_{\alpha, \varrho_{\alpha}}\right)\right)$ by [28, (83) and (84)], we get

$$
D_{\alpha}\left(\Lambda(f) \| \Lambda\left(\zeta_{\alpha, \varrho \wedge \varrho_{\alpha}}\right)\right) \leq C_{\alpha, \Lambda \varrho \wedge \varrho_{\alpha}}+\mathbb{1}_{\left\{\varrho<\varrho_{\alpha}\right\}} \frac{\varrho-\rho(f)}{b-a}\left[D_{\alpha}\left(\Lambda(a) \| \Lambda\left(\zeta_{\alpha, \varrho}\right)\right)-D_{\alpha}\left(\Lambda(b) \| \Lambda\left(\zeta_{\alpha, \varrho}\right)\right)\right] \quad \forall f \in \mathcal{F} .
$$

${ }^{8}$ Note that $C_{\alpha, \Lambda, \varrho}=C_{\alpha, \Lambda \leq \varrho}$ and $q_{\alpha, \Lambda, \varrho}=q_{\alpha, \Lambda \leq \varrho}$ for the Poisson Channel $\Lambda \leq \varrho:\{f \in \mathcal{F}: \rho(f) \leq \varrho\} \rightarrow \mathcal{P}(\mathcal{Y})$ considered in [28, Example 10]. 
On the other hand $D_{\alpha}\left(\Lambda(a) \| \Lambda\left(\zeta_{\alpha, \varrho}\right)\right) \leq D_{\alpha}\left(\Lambda(b) \| \Lambda\left(\zeta_{\alpha, \varrho}\right)\right)$ for all $\varrho \leq \varrho_{\alpha}$ by [28, (83)]; consequently, we have

$$
D_{\alpha}\left(\Lambda(f) \| \Lambda\left(\zeta_{\alpha, \varrho \wedge \varrho_{\alpha}}\right) \mid p\right) \leq C_{\alpha, \Lambda \varrho \wedge \varrho \alpha} \quad \forall p: \mathbf{E}_{p}[\rho] \leq \varrho .
$$

Thus (93) and (94) follow from [27, Lemma 25] because $\Lambda\left(\zeta_{\alpha, \varrho \wedge \varrho_{\alpha}}\right)=q_{\alpha, \Lambda \varrho \wedge \varrho_{\alpha}}$ by (86).

Since the expression for $C_{\alpha, \Lambda, \varrho}$ given in (93) is differentiable in $\alpha$, we can use the derivative test to determine optimal order for the SPE defined in (10). We obtain the following parametric form as a result

$$
\begin{aligned}
R & =\left(\frac{\varrho \wedge \varrho_{\alpha}-a}{b-a} b^{\alpha} \zeta_{\alpha, \varrho \wedge \varrho_{\alpha}}^{1-\alpha} \ln \frac{b^{\alpha} \zeta_{\alpha, \varrho \wedge \varrho_{\alpha}}^{1-\alpha}}{\zeta_{\alpha, \varrho \wedge \varrho_{\alpha}}}+\frac{b-\varrho \wedge \varrho_{\alpha}}{b-a} a^{\alpha} \zeta_{\alpha, \varrho \wedge \varrho_{\alpha}}^{1-\alpha} \ln \frac{a^{\alpha} \zeta_{\alpha, \varrho \wedge \varrho_{\alpha}}^{1-\alpha}}{\zeta_{\alpha, \varrho \wedge \varrho_{\alpha}}}\right) T \\
E_{s p}(R, \Lambda, \varrho) & =\left(\varrho \wedge \varrho_{\alpha}-\zeta_{\alpha, \varrho \wedge \varrho_{\alpha}}+\frac{\varrho \wedge \varrho_{\alpha}-a}{b-a} b^{\alpha} \zeta_{\alpha, \varrho \wedge \varrho_{\alpha}}^{1-\alpha} \ln \frac{b^{\alpha} \zeta_{\alpha, \varrho \wedge \varrho_{\alpha}}^{1-\alpha}}{b}+\frac{b-\varrho \wedge \varrho_{\alpha}}{b-a} a^{\alpha} \zeta_{\alpha, \varrho \wedge \varrho_{\alpha}}^{1-\alpha} \ln \frac{a^{\alpha} \zeta_{\alpha, \varrho \wedge \varrho_{\alpha}}^{1-\alpha}}{a}\right) T .
\end{aligned}
$$

Using [28, (68)], (86), (90), (94), (96), (97), we get the following parametric characterization

$$
\begin{aligned}
R & =D_{1}\left(\Lambda_{\alpha}^{q_{\alpha, \Lambda, \varrho}}\left(f_{\alpha}\right) \| q_{\alpha, \Lambda, \varrho}\right), \\
E_{s p}(R, \Lambda, \varrho) & =D_{1}\left(\Lambda_{\alpha, \Lambda, \varrho}^{q_{\alpha, \Lambda}}\left(f_{\alpha}\right) \| \Lambda\left(f_{\alpha}\right)\right),
\end{aligned}
$$

where $f_{\alpha}$ is any $\{a, b\}$ valued function in $\mathcal{F} \varrho \varrho_{\alpha}$, i.e. any function $f_{\alpha}:(0, T] \rightarrow\{a, b\}$ satisfying $\rho\left(f_{\alpha}\right)=\varrho \wedge \varrho_{\alpha}$.

\section{Discussion}

We have applied Augustin's method to derive SPBs for two families of memoryless channels. For the stationary memoryless channels with convex composition constraints, the novel observation behind Augustin's method is:

$$
\lim _{\phi \rightarrow \alpha} \sup _{p \in \mathcal{A}} D_{\alpha}\left(W \| q_{\phi, W, \mathcal{A}} \mid p\right)=C_{\alpha, W, \mathcal{A}} \quad \forall \alpha \in(0,1) .
$$

Note that the results established for the convex composition constrained stationary memoryless channels also hold for the cost constrained stationary memoryless channels because any cost constraint on a stationary memoryless channel can be expressed as a convex composition constraint, as well. For the non-stationary cost constrained memoryless channels we have employed (100) together with the convex conjugation techniques. Theorem 2 improves a similar result by Augustin, i.e. [25, Thm. 36.6], in terms of the approximation error terms. The prefactor of Theorem 2 is of the form $e^{-O(\ln n)}$, rather than $e^{-O(\sqrt{n})} \operatorname{similar}$ to [25, Thm. 36.6]. Also, unlike [25, Thm. 36.6], Theorem 2 does not assume the cost functions to be bounded and thus holds for the Gaussian models considered in [17]-[20], as well.

For classical-quantum channels, the SPB was established in [41]. Following this breakthrough, there has been a reviewed interest in the SPB for classical-quantum channels [42]-[45]. Augustin's method, however, has not been applied to any quantum information theoretic model. Successful applications of Augustin's method will allow us to get rid of the stationarity, and finite input set hypotheses, at the very least.

The Augustin's variant of Gallager's bound discussed in $\$ 2.4$ is not widely known. Our main aim in $\$ 2.4$ was to present this approach in its simplest form. Thus while bounding $\mathbf{E}\left[P_{\mathbf{e}}\right]$, we were content with passing from (29) to (30). Using more careful analysis and bounding the deviation of the order $\alpha$ A-L information random variable, i.e. $\ln \frac{f_{x}}{h_{x}}$ for $q=q_{\alpha, p}$, one can obtain sharper bounds similar to the ones in [46]-[48]. The random coding bound for the classical-quantum channels has been established in [49]. It is suggested in [43, p. 5606] that the reliance of [49] on the codes generated with i.i.d. symbols might make it hard to modify the proof to the constant composition, i.e. composition constrained, case. We think the Augustin's variant of Gallager's bound might be helpful in overcoming this issue.

As a side note, let us point out that $\S 2.4$ and $\S 3$ imply that SPE is the reliability function for certain fading channels, i.e. for certain channels with state, provided that the list decoding is allowed, even in the non-stationary case. In particular, both the fast fading channels with no state information (i.e. with statistical state information) and the fast fading channels with state information only at the receiver are cost constrained memoryless channels. Thus for the channels considered in [20] and the ones considered in [50, §4] the reliability function under list decoding is equal to the SPE. This is the case for the models with per antenna power constraints considered in [51]-[53], as well, because the channels considered in [51]-[53] are cost constrained memoryless channels albeit with multiple constraints. The determination of the SPE for these channels, however, is a separate issue, as we have noted in $\$ 4$.

The optimal prefactor of the SPB was known for specific channels since the early days of the information theory, see for example [3], [4], [17]. In recent years, there has been a reviewed interest in establishing such sharp SPBs under various symmetry hypothesis [5], [6], [13], [21]-[23]. The parametric characterization of the SPE given in (13) and (14) is used to establish such bounds for constant composition codes in [14]. A refined SPB can be established in every single one of the cases considered in [3]-[6], [13], [17], [21]-[23], using analogous parametric characterizations as demonstrated by [29]. This is one of the reasons for us to present the parametric characterizations given in (70), (71), (91), (92), (98), and (99). It turns out that one can strengthen the strong converses in terms of their prefactors using analogous parametric characterizations under appropriate symmetry hypothesis, as well, see [54]. 


\section{APPENDIX}

\section{A. Blahut's Approach}

In [32], Blahut derives a lower bound to the error probability of the channel codes without using constant composition arguments. Blahut claims that the exponential decay rate of his bound is equal to the SPE, see [32, Thm. 19]. Blahut claims the equality of the aforementioned exponent and the SPE in other publications too, see [33, Lemma 1] and [34, Thm. 10.1.4]. We show in the following that for the $Z$-channel the exponent of the Blahut's bound is infinite for any rate less than the channel capacity. Hence, [32, Thm. 19], [33, Lemma 1], and [34, Thm. 10.1.4] are all incorrect. More importantly, we show that even the best bound that can be obtained using Blahut's method is strictly inferior to the SPB in terms of its exponential decay rate with the block length.

For any $W: \mathcal{X} \rightarrow \mathcal{P}(\mathcal{Y})$ and $R \in\left[0, C_{1, W}\right]$, let $G(R, W, p, q)$ be

$$
G(R, W, p, q) \triangleq \inf _{V: D_{1}(V \| q \mid p) \leq R} D_{1}(V \| W \mid p) \quad \forall p \in \mathcal{P}(\mathcal{X}), q \in \mathcal{P}(\mathcal{Y}) .
$$

Since $D_{1}(V \|(p V) \mid p)=I_{1}(p ; V)$ where $(p V)=\sum_{x} p(x) V(x)$, the alternative expression for $E_{s p}(R, W)$ given in (20) implies

$$
E_{s p}(R, W)=\sup _{p \in \mathcal{P}(x)} \inf _{q \in \mathcal{P}(\mathcal{Y})} G(R, W, p, q) \quad \forall R \in\left[0, C_{1, W}\right] .
$$

Thus the max-min inequality implies

$$
E_{s p}(R, W) \leq \inf _{q \in \mathcal{P}(\mathcal{Y})} \sup _{p \in \mathcal{P}(\mathcal{X})} G(R, W, p, q) \quad \forall R \in\left[0, C_{1, W}\right] .
$$

The initial part of the proof of [32, Thm. 19] establishes the following bound on the error probability of the codes on a stationary product channel with the component channel $W$ whose input set $\mathcal{X}$ and output set $\mathcal{Y}$ are finite:

$$
P_{\mathbf{e}}^{\max , n} \geq O(1) e^{-o(n)-n \sup _{p \in \mathcal{P}(x)} G(R, W, p, q)} \quad \forall q \in \mathcal{P}(\mathcal{Y}) .
$$

The second half of the proof of [32, Thm. 19] claims that $\sup _{p \in \mathcal{P}(X)} G\left(R, W, p, q_{\alpha_{R}, p_{R}}\right)$ is equal to $E_{s p}(R, W)$ for some $\left(\alpha_{R}, p_{R}\right)$ pair satisfying the following equalities ${ }^{9}$

$$
\begin{aligned}
E_{s p}(R, W) & =E_{s p}\left(R, W, p_{R}\right), \\
& =\frac{1-\alpha_{R}}{\alpha_{R}}\left(I_{\alpha_{R}}\left(p_{R} ; W\right)-R\right) .
\end{aligned}
$$

When considered together with (A.1), the second half of the proof of [32, Thm. 19] asserts that

$$
\sup _{p \in \mathcal{P}(x)} G\left(R, W, p, q_{\alpha_{R}, p_{R}}\right) \stackrel{?}{=} \sup _{p \in \mathcal{P}(x)} \inf _{q \in \mathcal{P}(\mathcal{Y})} G(R, W, p, q) .
$$

[33, Lemma 1] and [34, Thm. 10.1.4] imply the same equality when considered together with (A.1), as well. In order to disprove (A.3), we consider the $Z$-channel, which is a discrete channel with the input set $X=\{1,2\}$ and the output set $y=\{a, b\}$ such that

$$
W=\left[\begin{array}{cc}
1 & 0 \\
\varepsilon & 1-\varepsilon
\end{array}\right] .
$$

We determine the order $\alpha$ Augustin capacity and the order $\alpha$ Augustin center using the identities $C_{\alpha, W}=D_{\alpha}\left(W(1) \| q_{\alpha, W}\right)$ and $C_{\alpha, W}=D_{\alpha}\left(W(2) \| q_{\alpha, W}\right)$ :

$$
\begin{aligned}
C_{\alpha, W} & =\ln \left(1+\left(\frac{1-\varepsilon^{\alpha}}{(1-\varepsilon)^{\alpha}}\right)^{\frac{1}{1-\alpha}}\right), \\
q_{\alpha, W}(a) & =\frac{(1-\varepsilon)^{\frac{\alpha}{1-\alpha}}}{(1-\varepsilon)^{\frac{\alpha}{1-\alpha}}+\left(1-\varepsilon^{\alpha}\right)^{\frac{1}{1-\alpha}}} .
\end{aligned}
$$

Then for $q=q_{\alpha, W}$ the tilted channel $W_{\alpha}^{q}$ defined in (3) is

$$
W_{\alpha}^{q_{\alpha, W}}=\left[\begin{array}{cc}
1 & 0 \\
\varepsilon^{\alpha} & 1-\varepsilon^{\alpha}
\end{array}\right]
$$

Furthermore, one can confirm by substitution that the order $\alpha$ Augustin center $q_{\alpha, W}$ is the fixed point of the order $\alpha$ Augustin operator defined in (4) for the prior $p_{\alpha, W}$ satisfying

$$
p_{\alpha, W}(1)=\frac{(1-\varepsilon)^{\frac{\alpha}{1-\alpha}}-\varepsilon^{\alpha}\left(1-\varepsilon^{\alpha}\right)^{\frac{\alpha}{1-\alpha}}}{(1-\varepsilon)^{\frac{\alpha}{1-\alpha}}+\left(1-\varepsilon^{\alpha}\right)^{\frac{1}{1-\alpha}}} .
$$

Thus the order $\alpha$ Augustin center $q_{\alpha, W}$ is equal to the order $\alpha$ Augustin mean for the prior $p_{\alpha, W}$, i.e. $q_{\alpha, W}=q_{\alpha, p_{\alpha, W}}$, by [27, Lemma13-(c,d)] and $I_{\alpha}\left(p_{\alpha, W} ; W\right)=D_{\alpha}\left(W \| q_{\alpha, W} \mid p_{\alpha, W}\right)$. Consequently $I_{\alpha}\left(p_{\alpha, W} ; W\right)=C_{\alpha, W}$, as well.

\footnotetext{
${ }^{9}$ Blahut mentions only the first equality explicitly.
} 
Note that $C_{\alpha, W}$ given in (A.4) is a differentiable function of $\alpha$ such that

$$
\begin{aligned}
\frac{\partial}{\partial \alpha} C_{\alpha, W} & =\frac{q_{\alpha}(b)}{(1-\alpha)^{2}}\left(\ln \frac{1-\varepsilon^{\alpha}}{1-\varepsilon}+\frac{\varepsilon^{\alpha}}{1-\varepsilon^{\alpha}} \ln \frac{\varepsilon^{\alpha}}{\varepsilon}\right), \\
& =\frac{1}{(1-\alpha)^{2}} D_{1}\left(W_{\alpha}^{q_{\alpha}, W} \| W \mid p_{\alpha, W}\right) .
\end{aligned}
$$

Then using first the identity $I_{\alpha}\left(p_{\alpha, W} ; W\right)=C_{\alpha, W}$ and then [27, (35)], we get

$$
\begin{aligned}
\frac{\partial}{\partial \alpha} \frac{1-\alpha}{\alpha}\left(C_{\alpha, W}-R\right) & =\frac{1}{\alpha^{2}}\left(R-C_{\alpha, W}+(1-\alpha) \alpha \frac{\partial}{\partial \alpha} C_{\alpha, W}\right) \\
& =\frac{1}{\alpha^{2}}\left(R-I_{\alpha}\left(p_{\alpha, W} ; W\right)+\frac{\alpha}{1-\alpha} D_{1}\left(W_{\alpha}^{q_{\alpha}, W} \| W \mid p_{\alpha, W}\right)\right) \\
& =\frac{1}{\alpha^{2}}\left(R-I_{1}\left(p_{\alpha, W} ; W_{\alpha}^{q_{\alpha, W}}\right)\right) .
\end{aligned}
$$

One can confirm numerically that $I_{1}\left(p_{\alpha, W} ; W_{\alpha}^{q_{\alpha}, W}\right)$ is increasing function of $\alpha$ for any $\varepsilon \in(0,1)$. Thus we can express the rate and the corresponding SPE in the following parametric form

$$
\begin{aligned}
R(\alpha) & =I_{1}\left(p_{\alpha, W} ; W_{\alpha}^{q_{\alpha}, W}\right) \\
E_{s p}(R(\alpha), W) & =D_{1}\left(W_{\alpha}^{q_{\alpha}, W} \| W \mid p_{\alpha, W}\right) .
\end{aligned}
$$

Thus for $R=R(\phi)$ we have $\left(\alpha_{R}, p_{R}\right)=\left(\phi, p_{\phi}, W\right)$ and $q_{\alpha_{R}, p_{R}}=q_{\phi, W}$. Then

$$
\begin{aligned}
\sup _{p \in \mathcal{P}(X)} G\left(R, W, p, q_{\alpha_{R}, p_{R}}\right) & \geq\left. G\left(R, W, p, q_{\alpha_{R}, W}\right)\right|_{p: p(1)=1} \cdot \\
& =\inf _{v: D_{1}\left(v \| q_{\alpha_{R}, W}\right) \leq R} D_{1}(v \| W(1))
\end{aligned}
$$

On the other hand, $v(b)>0$ for all $v$ satisfying $D_{1}\left(v \| q_{\alpha_{R}, W}\right) \leq R$ because $D_{1}\left(v \| q_{\alpha_{R}, W}\right)=\ln \frac{1}{q_{\alpha_{R}, W}(a)}=C_{\alpha_{R}}, W$ whenever $v(b)=0$ and $C_{\alpha_{R}, W}>R$. Furthermore, $D_{1}(v \| W(1))=\infty$ whenever $v(b)>0$. Thus

$$
\inf _{v: D_{1}\left(v \| q_{\alpha_{R}, W}\right) \leq R} D_{1}(v \| W(1))=\infty .
$$

Thus the bound established in the initial part of the proof of [32, Thm. 19], i.e. (A.2), is trivial for $q=q_{\alpha_{R}, p_{R}}$. Furthermore, [32, Thm. 19], [33, Lemma 1], and [34, Thm. 10.1.4] are all incorrect because $E_{s p}(R, W)<\infty$ for all $R \in\left[0, C_{1, W}\right]$ for the $Z$-channels. ${ }^{10}$

Using other $q$ 's one can obtain non-trivial bounds from (A.2); those bounds, however, do not imply the SPB either. If $q(a)<e^{-R}$ then $\sup _{p \in \mathcal{P}(X)} G\left(R, W, p, q_{\alpha_{R}, p_{R}}\right)=\infty$ as a result of the analysis presented above. We analyze the case $q(a) \geq e^{-R}$ in the following.

$$
\begin{aligned}
\sup _{p \in \mathcal{P}(x)} G(R, W, p, q) & \geq \inf _{V: D_{1}\left(V \| q \mid p_{R}\right) \leq R} D_{1}\left(V \| W \mid p_{R}\right) \\
& \geq \inf _{V} D_{1}\left(V \| W \mid p_{R}\right)+\frac{1-\alpha_{R}}{\alpha_{R}}\left(D_{1}\left(V \| q \mid p_{R}\right)-R\right) \\
& =\frac{1-\alpha_{R}}{\alpha_{R}}\left(D_{\alpha_{R}}\left(W \| q \mid p_{R}\right)-R\right) \\
& \geq \frac{1-\alpha_{R}}{\alpha_{R}}\left(I_{\alpha_{R}}\left(p_{R} ; W\right)+D_{\alpha_{R}}\left(q_{\alpha_{R}, p_{R}} \| q\right)-R\right) \\
& =E_{s p}(R, W)+\frac{1-\alpha_{R}}{\alpha_{R}} D_{\alpha_{R}}\left(q_{\alpha_{R}, p_{R}} \| q\right) \\
& \geq E_{s p}(R, W)+\frac{1-\alpha_{R}}{2}\left\|q-q_{\alpha_{R}, p_{R}}\right\|^{2}
\end{aligned}
$$

by $[27$, Lemma13-(c)],

by [37, Thm. 31].

On the other hand, $q_{\alpha_{R}, p_{R}}(a)<e^{-R}$ because $q_{\alpha_{R}, p_{R}}=q_{\alpha_{R}, W}$ and $C_{\alpha_{R}, W}>R$. Thus

$$
\begin{aligned}
\inf _{q \in \mathcal{P}(\mathcal{Y})} \sup _{p \in \mathcal{P}(X)} G(R, W, p, q) & \geq E_{s p}(R, W)+2\left(1-\alpha_{R}\right)\left(e^{-R}-q_{\alpha_{R}, W}(a)\right)^{2} \\
& =\sup _{p \in \mathcal{P}(X)} \inf _{q \in \mathcal{P}(\mathcal{Y})} G(R, W, p, q)+2\left(1-\alpha_{R}\right)\left(e^{-R}-q_{\alpha_{R}, W}(a)\right)^{2} .
\end{aligned}
$$

Hence, it is not possible to derive the SPB using Blahut's method, as it is presented in [32]. When the input set is finite, one can overcome this problem by employing composition based expurgations. But that approach had been presented by Haroutunian in [10], before [32].

\footnotetext{
${ }^{10}$ Recently, Yang argued that Blahut's method can be used to derive the SPB if the minimax equality given in [55, (3.63)] holds. Thus as a result of our analysis we can conclude that $[55,(3.63)]$ does not holds in general. This fact can be derived using the absence of the minimax equality for $G(R, W, p, q)$ without relying on the reasoning in [55], as well.
} 


\section{B. $R$-G Information Measures}

The order one R-G information measures are equal to the corresponding order one A-L information measures by definition. Thus our discussion will be confined to orders other than one.

Definition 12. For any $\alpha \in \mathbb{R}_{+} \backslash\{1\}$, channel $W: \mathcal{X} \rightarrow \mathcal{P}(\mathcal{Y})$ with a cost function $\rho: X \rightarrow \mathbb{R}_{\geq 0}^{\ell}, p \in \mathcal{P}(X)$, and $\lambda \in \mathbb{R}_{\geq 0}^{\ell}$ the order $\alpha$ Rényi-Gallager $(R-G)$ information for the input distribution $p$ and the Lagrange multiplier $\lambda$ is

$$
I_{\alpha}^{g \lambda}(p ; W) \triangleq \inf _{q \in \mathcal{P}(\mathcal{Y})} D_{\alpha}\left(p \circledast W e^{\frac{1-\alpha}{\alpha} \lambda \cdot \rho} \| p \otimes q\right) .
$$

If $\lambda$ is a vector of zeros, then the R-G information is the Rényi information. Similar to the Rényi information, the R-G information has a closed form expression, described in terms of a mean achieving the infimum in its definition.

Definition 13. For any $\alpha \in \mathbb{R}_{+}$, channel $W: \mathcal{X} \rightarrow \mathcal{P}(\mathcal{Y})$ with a cost function $\rho: \mathcal{X} \rightarrow \mathbb{R}_{\geq 0}^{\ell}, p \in \mathcal{P}(\mathcal{X})$, and $\lambda \in \mathbb{R}_{\geq 0}^{\ell}$, the order $\alpha$ mean measure for the input distribution $p$ and the Lagrange multiplier $\lambda$ is

$$
\frac{\mathrm{d} \mu_{\alpha, p}^{\lambda}}{\mathrm{d} \nu} \triangleq\left[\sum_{x} p(x) e^{(1-\alpha) \lambda \cdot \rho(x)}\left(\frac{\mathrm{d} W(x)}{\mathrm{d} \nu}\right)^{\alpha}\right]^{\frac{1}{\alpha}} .
$$

The order $\alpha$ Rényi-Gallager $(R-G)$ mean for the input distribution $p$ and the Lagrange multiplier $\lambda$ is

$$
q_{\alpha, p}^{g \lambda} \triangleq \frac{\mu_{\alpha, p}^{\lambda}}{\left\|\mu_{\alpha, p}^{\lambda}\right\|}
$$

Both $\mu_{\alpha, p}^{\lambda}$ and $q_{\alpha, p}^{g \lambda}$ depend on the Lagrange multiplier $\lambda$ for $\alpha \in \mathbb{R}_{+} \backslash\{1\}$. Furthermore, one can confirm by substitution that

$$
D_{\alpha}\left(p \circledast W e^{\frac{1-\alpha}{\alpha} \lambda \cdot \rho} \| p \otimes q\right)=I_{\alpha}^{g \lambda}(p ; W)+D_{\alpha}\left(q_{\alpha, p}^{g \lambda} \| q\right) \quad \alpha \in \mathbb{R}_{+} \backslash\{1\} .
$$

Then as a result of [27, Lemma 2] we have

$$
\begin{aligned}
& I_{\alpha}^{g \lambda}(p ; W)=D_{\alpha}\left(p \circledast W e^{\frac{1-\alpha}{\alpha} \lambda \cdot \rho} \| p \otimes q_{\alpha, W}^{g \lambda}\right) \\
& =\frac{\alpha}{\alpha-1} \ln \left\|\mu_{\alpha, p}^{\lambda}\right\| . \quad \alpha \in \mathbb{R}_{+} \backslash\{1\} .
\end{aligned}
$$

Using the definitions of the A-L information and the R-G information together with the Jensen's inequality and the concavity of the natural logarithm function we get

$$
\begin{aligned}
& I_{\alpha}^{\lambda}(p ; W) \geq I_{\alpha}^{g \lambda}(p ; W) \\
& \alpha \in(0,1] \\
& I_{\alpha}^{\lambda}(p ; W) \leq I_{\alpha}^{g \lambda}(p ; W) \\
& \alpha \in[1, \infty) \text {. }
\end{aligned}
$$

Definition 14. For any $\alpha \in \mathbb{R}_{+}$, channel $W: \mathcal{X} \rightarrow \mathcal{P}(\mathcal{Y})$ with a cost function $\rho: \mathcal{X} \rightarrow \mathbb{R}_{\geq 0}^{\ell}$, and $\lambda \in \mathbb{R}_{\geq 0}^{\ell}$, the order $\alpha$ Rényi-Gallager $(R-G)$ capacity for the Lagrange multiplier $\lambda$ is

$$
C_{\alpha, W}^{g \lambda} \triangleq \sup _{p \in \mathcal{P}(x)} I_{\alpha}^{g \lambda}(p ; W) .
$$

Although inequalities in (B.3) and (B.4) are strict for most input distributions, as a result of [27, Thm. 3], we have

$$
C_{\alpha, W}^{g \lambda}=S_{\alpha, W}^{\lambda} .
$$

Thus $C_{\alpha, W}^{g \lambda}=C_{\alpha, W}^{\lambda}$ by (9). This is the reason why in terms of determining the optimal performance either family can be used. The following lemma is, in essence, a restatement of [30, Thm. 8], which is the result that popularized the use of R-G information measures in cost constrained problems, see [18]-[20].

Lemma 12. For any $\ell, M, L \in \mathbb{Z}_{+}$s.t. $L<M, W: \mathcal{X} \rightarrow \mathcal{P}(\mathcal{Y}), \rho: \mathcal{X} \rightarrow \mathbb{R}_{\geq 0}^{\ell}, p \in \mathcal{P}(\mathcal{X}), \mathcal{B} \subset \mathcal{X}$, and $\alpha \in\left[\frac{1}{1+L}, 1\right)$ there exists an $(M, L)$ channel code with an encoding function of the form $\Psi: \mathcal{M} \rightarrow \mathcal{B}$ such that

$$
\ln P_{\mathbf{e}} \leq \frac{\alpha-1}{\alpha}\left[I_{\alpha}^{g \lambda}(p ; W)+\left(\inf _{x \in \mathcal{B}} \lambda \cdot \rho(x)\right)-\ln \frac{(M-1) e}{L}\right]-\frac{\ln p(\mathcal{B})}{\alpha} .
$$

Proof of Lemma 12. We follow the proof of Lemma 6 up to (30). As result of (26) and (30) we have

$$
\begin{aligned}
\ln \mathbf{E}\left[P_{\mathbf{e}}\right] & \leq \ln \mathbf{E}_{q}\left[\left(\sum_{x \in \mathcal{B}} \frac{p(x)}{p(\mathcal{B})} e^{(1-\alpha) \lambda \cdot \rho(x)}\left(\frac{\mathrm{d} W(x)}{\mathrm{d} q}\right)^{\alpha}\right)^{\frac{1}{\alpha}}\right]+\frac{\alpha-1}{\alpha}\left[\left(\inf _{x \in \mathcal{B}} \lambda \cdot \rho(x)\right)-\ln \frac{(M-1) e}{L}\right] \\
& \leq \ln \mathbf{E}_{q}\left[\left(\sum_{x \in X} p(x) e^{(1-\alpha) \lambda \cdot \rho(x)}\left(\frac{\mathrm{d} W(x)}{\mathrm{d} q}\right)^{\alpha}\right)^{\frac{1}{\alpha}}\right]+\frac{\alpha-1}{\alpha}\left[\left(\inf _{x \in \mathcal{B}} \lambda \cdot \rho(x)\right)-\ln \frac{(M-1) e}{L}\right]-\frac{\ln p(\mathcal{B})}{\alpha}
\end{aligned}
$$

Since there exists a code with $P_{\mathbf{e}}$ less than or equal to $\mathbf{E}\left[P_{\mathbf{e}}\right]$, the existence of a code satisfying (B.7) with an encoding function of the form $\Psi: \mathcal{M} \rightarrow \mathcal{B}$ follows from (B.1) and (B.2). 


\section{Omitted Proofs}

Proof of Lemma 1. $E_{s p}(R, W, \mathcal{A})$ is convex in $R$, because the pointwise supremum of a family of convex functions is convex and $\frac{1-\alpha}{\alpha}\left(C_{\alpha, W, \mathcal{A}}-R\right)$ is convex in $R$ for any $\alpha \in(0,1) . E_{s p}(R, W, \mathcal{A})$ is nonincreasing in $R$ as a result of an analogous argument. The continuity and finiteness claims are proved while establishing (11).

Recall that $C_{\alpha, W, \mathcal{A}}$ is a nondecreasing function of the order $\alpha$ by [27, Lemma 23-(a)].

- If $C_{0+, W, \mathcal{A}}=\infty$ then $C_{1 / 2, W}=\infty$ and $E_{s p}(R, W, \mathcal{A})=\infty$ for all $R \in \mathbb{R}_{\geq 0}$. On the other hand $R<C_{0+, W, \mathcal{A}}$ for all $R \in \mathbb{R}_{\geq 0}$. Hence (11) holds and claims about the continuity and the finiteness of $E_{s p}(R, W, \mathcal{A})$ are void.

- If $C_{0^{+}, W, \mathcal{A}}<\infty$ and $C_{0^{+}, W, \mathcal{A}}=C_{1, W, \mathcal{A}}$ then $E_{s p}(R, W, \mathcal{A})=\infty$ for all $R \in\left[0, C_{1, W, \mathcal{A}}\right)$ and $E_{s p}(R, W, \mathcal{A})=0$ for all $R \geq C_{1, W, \mathcal{A}}$. Hence (11) and claims about the continuity and the finiteness of $E_{s p}(R, W, \mathcal{A})$ hold.

- If $C_{0^{+}, W, \mathcal{A}}<\infty$ and $C_{0^{+}, W, \mathcal{A}} \neq C_{1, W, \mathcal{A}}$ then $E_{s p}(R, W, \mathcal{A})=\infty$ for all $R \in\left[0, C_{0^{+}, W, \mathcal{A}}\right)$. For $R \geq C_{0^{+}, W, \mathcal{A}}$, the non-negativity of $\frac{1-\alpha}{\alpha}\left(C_{\alpha, W, \mathcal{A}}-R\right)$ imply the restrictions given in (11).

As a result of $(11), E_{s p}(R, W, \mathcal{A})$ is finite for all $R>\lim _{\phi \downarrow} C_{\phi, W, \mathcal{A}}$. Thus, $E_{s p}(R, W, \mathcal{A})$ is continuous on $\left(C_{0^{+}, W, \mathcal{A}}, \infty\right)$ by [36, Thm. 6.3.3]. In order to extent the continuity to $\left[C_{0+, W, \mathcal{A}}, \infty\right)$, note that the function $\frac{1-\alpha}{\alpha}\left(C_{\alpha, W, \mathcal{A}}-R\right)$ is decreasing and continuous in $R$ for each $\alpha$ in $(0,1)$. Thus $E_{s p}(R, W, \mathcal{A})$ is a nonincreasing and lower semicontinuous function of $R$. Hence $E_{s p}(R, W, \mathcal{A})$ is continuous from the right and hence at $R=C_{0^{+}, W, \mathcal{A}}$.

Proof of Corollary 2. $\frac{1-\alpha}{\alpha}\left(C_{\alpha, W_{[1, n]}, n \varrho}-\ln \frac{M}{L}\right) \geq E_{s p}\left(\ln \frac{M}{L}, W_{[1, n]}, n \varrho\right)-\frac{1}{n}$ for an $\alpha \in(\eta, 1)$ by Lemma 1. There exists a $p \in \mathcal{P}\left(\widetilde{X}_{1}^{n}\right)$ of the form $p=\bigotimes_{t=1}^{n} p_{t}$ satisfying $\mathbf{E}_{p}\left[\rho_{[1, n]}\right] \leq n(\widetilde{\varrho}-\delta)$. Let $\widehat{\varrho} \triangleq \varrho-\frac{3 e \varsigma}{n}$. There exists a $\widetilde{p} \in \mathcal{P}\left(\widetilde{X}_{1}^{n}\right)$ of the form $\widetilde{p}=\bigotimes_{t=1}^{n} \widetilde{p}_{t}$ satisfying both $I_{\alpha}\left(\widetilde{p} ; \widetilde{W}_{[1, n]}\right) \geq C_{\alpha, \widetilde{W}_{[1, n]}, n \widehat{\varrho}}-\epsilon$ and $\mathbf{E}_{\widetilde{p}}\left[\rho_{[1, n]}\right] \leq n \widehat{\varrho}$ by [27, Lemmas 14 and 28]. Let $\widehat{W}_{[1, n]}: \widehat{X}_{1}^{n} \rightarrow \mathcal{P}\left(\mathcal{Y}_{1}^{n}\right)$ be a product channel satisfying $\widehat{W}_{[1, n]}\left(x_{1}^{n}\right)=W_{[1, n]}\left(x_{1}^{n}\right)$ for all $x_{1}^{n} \in \widehat{X}_{1}^{n}$ and $\widehat{X}_{t}=\operatorname{supp}\left(\widetilde{p}_{t}\right) \cup \operatorname{supp}\left(p_{t}\right)$

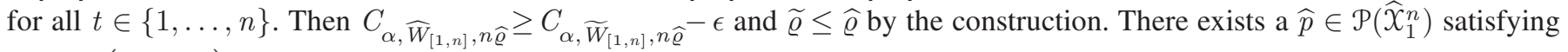
both $I_{\alpha}\left(\widehat{p} ; \widehat{W}_{[1, n]}\right)=C_{\alpha, \widehat{W}_{[1, n]}, n \widehat{\varrho}}$ and $\mathbf{E}_{\widehat{p}}\left[\rho_{[1, n]}\right] \leq n \widehat{\varrho}$ by [27, Lemma 19] because $\widehat{X}_{1}^{n}$ is finite. Furthermore, we can assume that $\widehat{p}$ is of the form $\widehat{p}=\bigotimes_{t=1}^{n} \widehat{p}_{t}$ without loss of generality by [27, Lemma 14] because the cost function $\rho_{[1, n]}$ is additive.

Note that $\mathbf{E}_{\widehat{p_{t}}}\left[\left|\rho_{t}-\mathbf{E}_{\widehat{p_{t}}}\left[\rho_{t}\right]\right|^{\kappa}\right]^{1 / \kappa} \leq \varsigma$ for all $\kappa \in \mathbb{R}_{+}$and $t \in\{1, \ldots, n\}$ because $\rho_{t}$ is positive and less than $\varsigma$ with probability one under $\widehat{p_{t}}$. Then using [26, Lemma 19] for $\kappa=\ln n$ we get

$$
\widehat{p}\left(\left|\rho_{[1, n]}(x)-\mathbf{E}_{\widehat{p}}\left[\rho_{[1, n]}\right]\right|<3 \varsigma e\right) \geq \frac{1}{2 \sqrt{n}} .
$$

On the other hand, there exists a $\widehat{\lambda}=\lambda_{\alpha, \widehat{W}_{[1, n]}, n \varrho}$ satisfying $C_{\alpha, \widehat{W}_{[1, n]}, n \widehat{\varrho}}=C_{\alpha, \widehat{W}_{[1, n]}}^{\widehat{\lambda}}+\widehat{\lambda} n \widehat{\varrho}$ by [27, Lemma 29-(c)] because $n \widehat{\varrho}$ is in the interior of the feasible cost constraints $\widehat{W}_{[1, n]}$ by construction. Furthermore, $I_{\alpha}^{\widehat{\lambda}}\left(\widehat{p} ; \widehat{W}_{[1, n]}\right)=C_{\alpha, \widehat{W}_{[1, n]}}$ by $[27$, Lemma 29-(d)] because $I_{\alpha}\left(\widehat{p} ; \widehat{W}_{[1, n]}\right)=C_{\alpha, \widehat{W}_{[1, n]}, n \widehat{\varrho}}$ and $\mathbf{E}_{\widehat{p}}\left[\rho_{[1, n]}\right] \leq n \widehat{\varrho}$. As a result $D_{\alpha}\left(W_{[1, n]}\left(x_{1}^{n}\right) \| q_{\alpha, \widehat{p}}\right)-\widehat{\lambda} \rho_{[1, n]}\left(x_{1}^{n}\right)=C_{\alpha, \widehat{W}_{[1, n]}}$ for all $x_{1}^{n} \in \operatorname{supp}(\widehat{p})$ and $\widehat{\lambda} n \widehat{\varrho}=\widehat{\lambda} \mathbf{E}_{\widehat{p}}\left[\rho_{[1, n]}\right]$. Applying Lemma 6 for $\mathcal{B}=\left\{x_{1}^{n} \in \operatorname{supp}(\widehat{p}):\left|\rho_{[1, n]}\left(x_{1}^{n}\right)-\mathbf{E}_{\widehat{p}}\left[\rho_{[1, n]}\right]\right|<3 \varsigma e\right\}$ we can conclude that there exists an $(M, L)$ channel code satisfying

$$
\begin{aligned}
\ln P_{\mathbf{e}} & \leq \frac{\alpha-1}{\alpha}\left[C_{\alpha, \widehat{W}_{[1, n]}}^{\widehat{\lambda}}+\inf _{x_{1}^{n} \in \mathcal{B}} \widehat{\lambda} \rho_{[1, n]}\left(x_{1}^{n}\right)-\ln \frac{(M-1) e}{L}\right]+\frac{\ln 4 n}{2 \alpha} \\
& \leq \frac{\alpha-1}{\alpha}\left[C_{\alpha, \widehat{W}_{[1, n]}, n \widehat{\varrho}}-3 \varsigma e \widehat{\lambda}-\ln \frac{(M-1) e}{L}\right]+\frac{\ln 4 n}{2 \alpha} .
\end{aligned}
$$

Since $C_{\alpha, \widehat{W}_{[1, n]}, n \widehat{\varrho}}+\widehat{\lambda} n(\varrho-\widehat{\varrho}) \geq C_{\alpha, \widehat{W}_{[1, n]}, n \varrho}$ by [27, Lemma 27-(b)] we get

$$
\ln P_{\mathbf{e}} \leq-E_{s p}\left(\ln \frac{M}{L}, W_{[1, n]}, \varrho\right)+\frac{1}{n}+\frac{1-\alpha}{\alpha}(6 \varsigma e \widehat{\lambda}+2 \epsilon+1)+\frac{\ln 4 n}{2 \alpha} .
$$

Then (25) holds because $\frac{1-\alpha}{\alpha} C_{\alpha, W_{[1, n]}, n \varrho}$ is nonincreasing in $\alpha$ by [27, Lemma 23-(b)], provided that $\widehat{\lambda} \leq \frac{C_{\alpha, W_{[1, n]}, n \varrho}}{n \delta}$. In order to see why such a bound holds first note that $\widehat{\varrho} \leq \varrho$ by definition and thus $C_{\alpha, \widehat{W}_{[1, n]}, n \varrho} \leq C_{\alpha, \widehat{W}_{[1, n]}, n \varrho}$. Furthermore,

$$
\begin{aligned}
C_{\alpha, \widehat{W}_{[1, n]}, n \widehat{\varrho}} & =\inf _{\lambda \geq \widehat{\lambda}} C_{\alpha, \widehat{W}_{[1, n]}^{\lambda}}+\lambda n \widehat{\varrho} \\
& >n \delta \widehat{\lambda}+\inf _{\lambda \geq \widehat{\lambda}} C_{\alpha, \widehat{W}_{[1, n]}}^{\lambda}+n \lambda(\widehat{\varrho}-\delta) \\
& \geq n \delta \widehat{\lambda}+C_{\alpha, \widehat{W}_{[1, n]}, n(\widehat{\varrho}-\delta)} \\
& \geq n \delta \widehat{\lambda}
\end{aligned}
$$

Proof of Lemma 8. $C_{\alpha, W, \mathcal{A}} \leq \widetilde{C}_{\alpha, W, \mathcal{A}}^{\epsilon}$ by (39) and [27, Lemma 23-(a,b)]. Then as a result of the expressions for $E_{s p}(R, W, \mathcal{A})$ given in (11) and the definition of $\widetilde{E}_{s p}^{\epsilon}(R, W, \mathcal{A})$ given in (40) we have

$$
E_{s p}(R, W, \mathcal{A}) \leq \widetilde{E}_{s p}^{\epsilon}(R, W, \mathcal{A}) \quad \forall R \in \mathbb{R}_{\geq 0} .
$$


Let us proceed with bounding $\widetilde{E}_{s p}^{\epsilon}(R, W, \mathcal{A})-E_{s p}(R, W, \mathcal{A})$ from above for $R \in\left[C_{\phi, W, \mathcal{A}}, \infty\right)$.

$$
\begin{aligned}
\frac{1-\alpha}{\alpha}\left(\widetilde{C}_{\alpha, W, \mathcal{A}}^{\epsilon}-R\right) & =\frac{1}{\epsilon} \int_{\alpha-\epsilon \alpha}^{\alpha+\epsilon(1-\alpha)}\left(\frac{1-\alpha}{\alpha} \vee \frac{1-\eta}{\eta}\right) C_{\eta, W, \mathcal{A}} \mathrm{d} \eta-\frac{1-\alpha}{\alpha} R \\
& =\frac{1}{\epsilon} \frac{1-\alpha}{\alpha} \int_{\alpha}^{\alpha+\epsilon(1-\alpha)}\left(C_{\eta, W, \mathcal{A}}-R\right) \mathrm{d} \eta+\frac{1}{\epsilon} \int_{\alpha-\epsilon \alpha}^{\alpha} \frac{1-\eta}{\eta}\left(C_{\eta, W, \mathcal{A}}-R\right) \mathrm{d} \eta+\frac{R}{\epsilon} \int_{\alpha-\epsilon \alpha}^{\alpha} \frac{\alpha-\eta}{\eta \alpha} \mathrm{d} \eta \\
& \leq \frac{1}{\epsilon} \frac{1-\alpha}{\alpha} \int_{\alpha}^{\alpha+\epsilon(1-\alpha)}\left(C_{\eta, W, \mathcal{A}}-R\right) \mathrm{d} \eta+\frac{1}{\epsilon} \int_{\alpha-\epsilon \alpha}^{\alpha} \frac{1-\eta}{\eta}\left(C_{\eta, W, \mathcal{A}}-R\right) \mathrm{d} \eta+\frac{\epsilon}{1-\epsilon} R
\end{aligned}
$$

We bound $\widetilde{E}_{s p}^{\epsilon}(R, W, \mathcal{A})$ by bounding the expression in (C.2) separately on two intervals for $\alpha$.

In order to bound the expression in (C.2) for $\alpha \in[\phi, 1)$, we use the fact that $\sup _{\eta \in(0,1)} \frac{1-\eta}{\eta}\left(C_{\eta}, W, \mathcal{A}-R\right)=E_{s p}(R, W, \mathcal{A})$.

$$
\begin{aligned}
\frac{1-\alpha}{\alpha}\left(\widetilde{C}_{\alpha, W, \mathcal{A}}^{\epsilon}-R\right) & \leq \frac{1}{\epsilon} \frac{1-\alpha}{\alpha} \int_{\alpha}^{\alpha+\epsilon(1-\alpha)}\left(C_{\eta, W, \mathcal{A}}-R\right) \mathrm{d} \eta+\frac{1}{\epsilon} \int_{\alpha-\epsilon \alpha}^{\alpha} \frac{1-\eta}{\eta}\left(C_{\eta, W, \mathcal{A}}-R\right) \mathrm{d} \eta+\frac{\epsilon}{1-\epsilon} R \\
& \leq \frac{1}{\epsilon} \frac{1-\alpha}{\alpha} \int_{\alpha}^{\alpha+\epsilon(1-\alpha)} \frac{\eta}{1-\eta} E_{s p}(R, W, \mathcal{A}) \mathrm{d} \eta+\frac{1}{\epsilon} \int_{\alpha-\epsilon \alpha}^{\alpha} E_{s p}(R, W, \mathcal{A}) \mathrm{d} \eta+\frac{\epsilon}{1-\epsilon} R \\
& \leq E_{s p}(R, W, \mathcal{A})+\frac{\epsilon}{1-\epsilon} \frac{1-\alpha}{\alpha} E_{s p}(R, W, \mathcal{A})+\frac{\epsilon}{1-\epsilon} R .
\end{aligned}
$$

Since $(1-\alpha) E_{s p}(R, W, \mathcal{A})+\alpha R \leq\left(R \vee E_{s p}(R, W, \mathcal{A})\right)$ for all $\alpha \in(0,1)$, we have

$$
\frac{1-\alpha}{\alpha}\left(\widetilde{C}_{\alpha, W, \mathcal{A}}^{\epsilon}-R\right) \leq E_{s p}(R, W, \mathcal{A})+\frac{\epsilon}{1-\epsilon} \frac{R \vee E_{s p}(R, W, \mathcal{A})}{\phi} \quad \alpha \in[\phi, 1)
$$

In order to bound the expression in (C.2) for $\alpha \in(0, \phi]$, recall that $C_{\alpha, W, \mathcal{A}}$ is nondecreasing in $\alpha$ by [27, Lemma 23-(a)]. Thus for any $R \geq C_{\phi, W, \mathcal{A}}$ we have

$$
\begin{aligned}
\frac{1-\alpha}{\alpha}\left(\widetilde{C}_{\alpha, W, \mathcal{A}}^{\epsilon}-R\right) & \leq \frac{1}{\epsilon} \frac{1-\alpha}{\alpha} \int_{\phi}^{\alpha+\epsilon(1-\alpha)} \frac{\eta}{1-\eta} E_{s p}(R, W, \mathcal{A}) \mathrm{d} \eta \mathbb{1}_{\left\{\alpha \in\left[\frac{\phi-\epsilon}{1-\epsilon}, \phi\right]\right\}}+\frac{\epsilon}{1-\epsilon} R \\
& \leq \frac{1}{\epsilon} \frac{\alpha+\epsilon(1-\alpha)}{\alpha(1-\epsilon)} \int_{\phi}^{\alpha+\epsilon(1-\alpha)} E_{s p}(R, W, \mathcal{A}) \mathrm{d} \eta \mathbb{1}_{\left\{\alpha \in\left[\frac{\phi-\epsilon}{1-\epsilon}, \phi\right]\right\}}+\frac{\epsilon}{1-\epsilon} R \\
& =\left[\frac{\alpha(1-\epsilon)+2 \epsilon-\phi}{\epsilon}-\frac{\phi-\epsilon}{\alpha(1-\epsilon)}\right] E_{s p}(R, W, \mathcal{A}) \mathbb{1}_{\left\{\alpha \in\left[\frac{\phi-\epsilon}{1-\epsilon}, \phi\right]\right\}}+\frac{\epsilon}{1-\epsilon} R \quad \alpha \in(0, \phi] . \\
& \leq(1-\phi) E_{s p}(R, W, \mathcal{A})+\frac{\epsilon}{1-\epsilon} \frac{(1-\phi) E_{s p}(R, W, \mathcal{A})+\phi R}{\phi} \quad \alpha \quad
\end{aligned}
$$

(41) follows from (C.1), (C.3), and (C.4).

On the other hand $C_{\alpha, W, \mathcal{A}}$ is nondecreasing and $\frac{1-\alpha}{\alpha} C_{\alpha, W, \mathcal{A}}$ is nonincreasing in $\alpha$ by [27, Lemma 23-(a,b)]. Then as a result of the expression for $E_{s p}(R, W, \mathcal{A})$ given in $(11)$, we have $E_{s p}(R, W, \mathcal{A}) \leq \frac{1-\phi}{\phi} R$ for all $R \in\left[C_{\phi}, W, \mathcal{A}, \infty\right)$. Hence,

$$
R \vee E_{s p}(R, W, \mathcal{A}) \leq R / \phi \quad \forall R \in\left[C_{\phi, W, \mathcal{A}}, \infty\right) .
$$

(42) follows from (41) and (C.5).

Proof of Theorem 1. We prove Theorem 1 using Lemmas 8 and 9. We are free to choose different values for $\kappa$ and $\epsilon$ for different values of $n$, provided that the hypotheses of Lemmas 8 and 9 are satisfied.

As a result of Assumption 1 there exists a $K \in[1, \infty)$ and an $n_{0} \in \mathbb{Z}_{+}$such that

$$
\max _{t: t \leq n} C_{1 / 2, U_{\mathcal{A}}^{(t)}} \leq K \ln (n) \quad \forall n \geq n_{0}
$$

Let $\kappa_{n}$ be $\kappa_{n}=K \ln (1+n)$. Then

$$
\gamma_{n} \leq 40(K+1) \ln (1+n) \quad \forall n \geq n_{0} .
$$

$C_{\alpha, W, \mathcal{A}}$ is nondecreasing in $\alpha$ by [27, Lemma 23-(a)] and $\frac{1-\alpha}{\alpha} C_{\alpha, W, \mathcal{A}}$ is nonincreasing in $\alpha$ on $(0,1)$ by [27, Lemma 23-(b)]. Thus, we can bound $\widetilde{C}_{\alpha, W, \mathcal{A}}^{\epsilon}$ using its definition given in (39):

$$
\begin{aligned}
\widetilde{C}_{\alpha, W, \mathcal{A}}^{\epsilon} & =\frac{1}{\epsilon} \int_{\alpha-\epsilon \alpha}^{\alpha+\epsilon(1-\alpha)}\left[1 \vee\left(\frac{\alpha}{1-\alpha} \frac{1-\eta}{\eta}\right)\right] C_{\eta, W, \mathcal{A}} \mathrm{d} \eta \\
& \leq \frac{1}{\epsilon} \int_{\alpha-\epsilon \alpha}^{\alpha+\epsilon(1-\alpha)}\left[1 \vee\left(\frac{\alpha}{1-\alpha} \frac{1-\eta}{\eta}\right)\right]\left[1 \vee\left(\frac{1-\alpha}{\alpha} \frac{\eta}{1-\eta}\right)\right] C_{\alpha, W, \mathcal{A}} \mathrm{d} \eta \\
& =\frac{C_{\alpha, W, \mathcal{A}}}{\epsilon} \int_{\alpha-\epsilon \alpha}^{\alpha+\epsilon(1-\alpha)}\left[\left(\frac{\alpha}{1-\alpha} \frac{1-\eta}{\eta}\right) \vee\left(\frac{1-\alpha}{\alpha} \frac{\eta}{1-\eta}\right)\right] \mathrm{d} \eta \\
& \leq\left(1+\frac{\epsilon}{1-\epsilon} \frac{\alpha^{2}+(1-\alpha)^{2}}{\alpha(1-\alpha)}\right) C_{\alpha, W, \mathcal{A}}
\end{aligned}
$$


Then for $\epsilon_{n}=1 / n,($ C.6) imply that

$$
n \widetilde{C}_{\phi, W, \mathcal{A}}^{\epsilon}+\frac{\gamma_{n}}{1-\phi}+\ln \frac{8 e^{3} n^{1.5}}{\epsilon} \leq n C_{\phi, W, \mathcal{A}}+\frac{n}{n-1} \frac{C_{\phi, W, \mathcal{A}}}{\phi(1-\phi)}+\frac{40(K+1) \ln (1+n)}{1-\phi}+3 \ln (2 e n) \quad \forall n \geq n_{0} .
$$

Thus the hypothesis of Theorem 1 implies the hypothesis of Lemma 9 for all $n$ large enough. Consequently, for all $n$ large enough Lemma 9 and (C.6) implies that

$$
P_{\mathbf{e}} \geq\left(\frac{(1+n)^{-80(K+1)}}{8 e^{3} n^{2.5}}\right)^{1 / \phi} e^{-n \widetilde{E}_{s p}^{\epsilon_{n}}\left(\frac{1}{n} \ln \frac{M_{n}}{L_{n}}, W, \mathcal{A}\right)} .
$$

On the other hand, Lemma 8, (31), and the monotonicity of $C_{\alpha, W, \mathcal{A}}$ in $\alpha$ imply that for $n$ large enough

$$
\widetilde{E}_{s p}^{\epsilon_{n}}\left(\frac{1}{n} \ln \frac{M_{n}}{L_{n}}, W, \mathcal{A}\right) \leq E_{s p}\left(\frac{1}{n} \ln \frac{M_{n}}{L_{n}}, W, \mathcal{A}\right)+\frac{C_{\alpha_{1}, W, \mathcal{A}}}{(n-1) \phi^{2}} .
$$

(32) follows from (C.8) and (C.9).

Proof of Theorem 2. We prove Theorem 2 using Lemmas 8 and 10. We are free to choose different values for $\kappa$ and $\epsilon$ for different values of $n$, provided that the hypotheses of Lemmas 8 and 10 are satisfied.

As a result of Assumption 2 there exists a $K \in[1, \infty)$ and an $n_{0} \in \mathbb{Z}_{+}$such that

$$
\max _{t: t \leq n} C_{1 / 2, W_{t}, n \varrho} \leq K \ln n \quad \forall n \geq n_{0} .
$$

Let $\kappa_{n}$ be $\kappa_{n}=K \ln (1+n)$. Then

$$
\gamma_{n} \leq 40(K+1) \ln (1+n) \quad \forall n \geq n_{0} .
$$

$C_{\alpha, W, \varrho}$ is nondecreasing in $\alpha$ by [27, Lemma 23-(a)] and $\frac{1-\alpha}{\alpha} C_{\alpha, W, \varrho}$ is nonincreasing in $\alpha$ on (0,1) by [27, Lemma 23-(b)]. Thus, we can bound $\widetilde{C}_{\alpha, W, \varrho}^{\epsilon}$ using its definition given in (39):

$$
\widetilde{C}_{\alpha, W_{[1, n]}, n \varrho}^{\epsilon} \leq\left(1+\frac{\epsilon}{1-\epsilon} \frac{\alpha^{2}+(1-\alpha)^{2}}{\alpha(1-\alpha)}\right) C_{\alpha, W_{[1, n]}, n \varrho .}
$$

Then for $\epsilon_{n}=\frac{1}{n}$, (C.10), (C.11), and [27, Lemmas 23-(a,b), 27-(a), 28] imply that

$$
\widetilde{C}_{\alpha, W_{[1, n]}, n \varrho}^{\epsilon}+\frac{\gamma_{n}}{1-\phi}+\ln \frac{8 e^{3} n^{1.5}}{\epsilon} \leq C_{\alpha, W_{[1, n]}, n \varrho}+\frac{n}{n-1} \frac{K \ln (n)}{\phi(1-\phi)}+\frac{40(K+1) \ln (1+n)}{1-\phi}+3 \ln (2 e n) \quad \forall n \geq n_{0} .
$$

Thus the hypothesis of Theorem 2 implies the hypothesis of Lemma 10 for all $n$ large enough. Consequently, for all $n$ large enough Lemma 10 and (C.11) implies that

$$
P_{\mathbf{e}} \geq\left(\frac{(1+n)^{-80(K+1)}}{8 e^{3} n^{2.5}}\right)^{1 / \phi} e^{-\widetilde{E}_{s p}^{\epsilon_{n}}\left(\ln \frac{M_{n}}{L_{n}}, W_{[1, n]}, n \varrho\right)} .
$$

On the other hand, Lemma 8, (33), and the monotonicity of $C_{\alpha, W, \varrho}$ in $\alpha$ imply that for $n$ large enough

$$
\widetilde{E}_{s p}^{\epsilon_{n}}\left(\ln \frac{M_{n}}{L_{n}}, W_{[1, n]}, n \varrho\right) \leq E_{s p}\left(\ln \frac{M_{n}}{L_{n}}, W_{[1, n]}, n \varrho\right)+\frac{C_{\alpha_{1}, W_{[1, n]}, n \varrho}}{(n-1) \phi^{2}} .
$$

Note that $C_{\alpha, W_{[1, n]}, n \varrho}$ is nondecreasing in $\alpha$ by [27, Lemma 23-(a)] and $\frac{1-\alpha}{\alpha} C_{\alpha, W_{[1, n]}, n \varrho}$ is nonincreasing in $\alpha$ on $(0,1)$ by [27, Lemma 23-(b)]. Thus

$$
C_{\alpha_{1}, W_{[1, n]}, n \varrho} \leq\left(\frac{\alpha_{1}}{1-\alpha_{1}} \vee 1\right) C_{1 / 2, W_{[1, n]}, n \varrho} .
$$

Since $C_{\alpha, W, \varrho}$ is non-decreasing in $\varrho$ by [27, Lemmas 27-(a)], (C.10) and [27, Lemma 28] imply for all $n$ large enough

$$
C_{1 / 2, W_{[1, n]}, n \varrho \leq K n \ln n} .
$$

For $n$ large enough (C.14), (C.15), and (C.16) imply

$$
\widetilde{E}_{s p}^{\epsilon_{n}}\left(\ln \frac{M_{n}}{L_{n}}, W_{[1, n]}, n \varrho\right) \leq E_{s p}\left(\ln \frac{M_{n}}{L_{n}}, W_{[1, n]}, n \varrho\right)+\frac{2}{\phi^{2}}\left(\frac{\alpha_{1}}{1-\alpha_{1}} \vee 1\right) K \ln n .
$$

(34) follows from (C.13) and (C.17).

\section{ACKNOWLEDGMENT}

The author would like to thank Fatma Nakiboğlu and Mehmet Nakiboğlu for their hospitality; this work would not have been possible without it. The author would like to thank Marco Dalai for informing him about Fano's implicit assertion of the fixed point property in [9], Gonzalo Vazquez-Vilar for pointing out Poltyrev's paper [31] on the random coding bound and for his suggestions on the manuscript, Wei Yang for pointing out [55, (3.63)] and its relation to Blahut's approach, and the reviewer his suggestions on the manuscript. 


\section{REFERENCES}

[1] B. Nakiboğlu. The Augustin center and the sphere packing bound for memoryless channels. In 2017 IEEE International Symposium on Information Theory (ISIT), pages 1401-1405, Aachen, Germany, June 2017.

[2] P. Elias. Coding for noisy channels. In IRE Convention Record 3(4), pages 37-46, 1955. (reprinted in Key Papers in the Development of Coding Theory, ed. E. Berlekamp, pp. 48-55. New York:IEEE Press, 1974.).

[3] P. Elias. Coding for two noisy channels. In Proceedings of Third London Symposium of Information Theory, pages 61-74, London, 1955. Butterworth Scientific.

[4] R. Dobrushin. Asymptotic estimates of the probability of error for transmission of messages over a discrete memoryless communication channel with a symmetric transition probability matrix. Theory of Probability \& Its Applications, 7(3):270-300, 1962.

[5] Y. Altuğ and A. B. Wagner. On exact asymptotics of the error probability in channel coding: symmetric channels. arXiv:1908.11419 [cs.IT], 2019.

[6] Y. Altuğ and A. B. Wagner. Refinement of the sphere packing bound for symmetric channels. In 49th Annual Allerton Conference on Communication, Control, and Computing, pages 30-37, Sept 2011.

[7] C. E. Shannon, R. G. Gallager, and E. R. Berlekamp. Lower bounds to error probability for coding on discrete memoryless channels. I. Information and Control, 10(1):65-103, 1967.

[8] Robert G. Gallager. Information theory and reliable communication. John Wiley \& Sons, Inc., New York, NY, 1968.

[9] Roberto M. Fano. Transmission of Information: A Statistical Theory of Communications. M.I.T. Press, Cambridge, MA, 1961.

[10] E. A. Haroutunian. Estimates of the error probability exponent for a semicontinuous memoryless channel. Problems of Information Transmission, 4(4):37-48, 1968

[11] J. K. Omura. A lower bounding method for channel and source coding probabilities. Information and Control, 27(2):148 - 177, 1975.

[12] Imre Csiszár and János Körner. Information theory: coding theorems for discrete memoryless systems. Cambridge University Press, Cambridge, UK, 2011.

[13] Y. Altuğ and A. B. Wagner. Refinement of the sphere-packing bound: Asymmetric channels. IEEE Transactions on Information Theory, 60(3):1592-1614, March 2014

[14] B. Nakiboğlu. A simple derivation of the refined spb for the constant composition codes. In 2019 IEEE International Symposium on Information Theory (ISIT), pages 2659-2663, Paris, France, July 2019.

[15] A. D. Wyner. Capacity and error exponent for the direct detection photon channel. II. IEEE Transactions on Information Theory, 34(6):1462-1471, Nov 1988.

[16] M. V. Burnashev and Yu. A. Kutoyants. On the sphere-packing bound, capacity, and similar results for Poisson channels. Problems of Information Transmission, 35(2):95-111, 1999.

[17] C. E. Shannon. Probability of error for optimal codes in a Gaussian channel. The Bell System Technical Journal, 38(3):611-656, May 1959.

[18] P. M. Ebert. Error bounds for gaussian noise channels. Quarterly Progress Report, 77(B):292-302, 1965. (http://hdl.handle.net/1721.1/55609).

[19] Paul Michael Ebert. Error Bounds For Parallel Communication Channels. Technical report 448, Research Laboratory of Electronics at Massachusetts Institute of Technology, Cambridge, MA, 1966. (http://hdl.handle.net/1721.1/4295).

[20] John Stephen Richters. Communication over fading dispersive channels. Technical report 464, Research Laboratory of Electronics at Massachusetts Institute of Technology, Cambridge, MA, 1967. (http://hdl.handle.net/1721.1/4279).

[21] Alejandro Lancho Serrano. Fundamental limits of short-packet wireless communications. Ph.D. Thesis, Universidad Carlos III de Madrid, Departamento de Teoria de la Senal y Comunicaciones, June 2019. (https://e-archivo.uc3m.es/handle/10016/29596).

[22] A. Lancho, J. Östman, G. Durisi, T. Koch, and G. Vazquez-Vilar. Saddlepoint approximations for noncoherent single-antenna rayleigh block-fading channels. In 2019 IEEE International Symposium on Information Theory (ISIT), pages 612-616, July 2019.

[23] A. Lancho, J. Östman, G. Durisi, T. Koch, and G. Vazquez-Vilar. Saddlepoint approximations for short-packet wireless communications. arXiv:1904.10442 [cs.IT], 2019.

[24] U. Augustin. Error estimates for low rate codes. Zeitschrift für Wahrscheinlichkeitstheorie und Verwandte Gebiete, 14(1):61-88, 1969.

[25] Udo Augustin. Noisy Channels. Habilitation thesis, Universität Erlangen-Nürnberg, 1978. (http://bit.ly/2ID8h7m).

[26] B. Nakiboğlu. The sphere packing bound via Augustin's method. IEEE Transactions on Information Theory, 65(2):816-840, Feb 2019. (arXiv:1611.06924 [cs.IT]).

[27] B. Nakiboğlu. The Augustin capacity and center. Problems of Information Transmission, 55(4):299-342, October 2019. (arXiv:1803.07937 [cs.IT]).

[28] B. Nakiboğlu. The Rényi capacity and center. IEEE Transactions on Information Theory, 65(2):841-860, Feb 2019. (arXiv:1608.02424 [cs.IT]).

[29] B. Nakiboğlu. A simple derivation of the refined SPB under certain symmetry hypotheses. arXiv:1904.12780v2, 2019.

[30] R. G. Gallager. A simple derivation of the coding theorem and some applications. IEEE Transactions on Information Theory, 11(1):3-18, Jan. 1965.

[31] G. Sh. Poltyrev. Random coding bounds for discrete memoryless channels. Problems of Information Transmission, 18(1):9-21, 1982.

[32] R. E. Blahut. Hypothesis testing and information theory. IEEE Transactions on Information Theory, 20(4):405-417, Jul 1974.

[33] R. Blahut. Information bounds of the Fano-Kullback type. IEEE Transactions on Information Theory, 22(4):410-421, Jul 1976.

[34] Richard E. Blahut. Principles and Practice of Information Theory. Addison-Wesley, Reading, MA, 1987.

[35] G. Vazquez-Vilar, A. Martinez, and A. G. i Fabregas. A derivation of the cost-constrained sphere-packing exponent. In 2015 IEEE International Symposium on Information Theory, pages 929-933, Hong Kong, P.R.C., June 2015.

[36] Richard M. Dudley. Real analysis and probability, volume 74. Cambridge University Press, New York, NY, 2002.

[37] T. van Erven and P. Harremoës. Rényi divergence and Kullback-Leibler divergence. IEEE Transactions on Information Theory, 60(7):3797-3820, July 2014.

[38] Walter Rudin. Principles of Mathematical Analysis. McGraw-Hill, New York, NY, 1976.

[39] Vladimir I. Bogachev. Measure Theory. Springer-Verlag, Berlin Heidelberg, 2007.

[40] P. Elias. List decoding for noisy channels. Technical report 335, Research Laboratory of Electronics at Massachusetts Institute of Technology, Cambridge, MA, 1957. (http://hdl.handle.net/1721.1/4484)

[41] M. Dalai. Lower bounds on the probability of error for classical and classical-quantum channels. IEEE Transactions on Information Theory, 59(12):80278056 , Dec 2013.

[42] M. Dalai. Some remarks on classical and classical-quantum sphere packing bounds: Rényi vs. Kullback-Leibler. Entropy, $19(7): 355,2017$.

[43] M. Dalai and A. Winter. Constant compositions in the sphere packing bound for classical-quantum channels. IEEE Transactions on Information Theory, 63(9):5603-5617, Sept 2017.

[44] H. C. Cheng and M. H. Hsieh. Moderate deviation analysis for classical-quantum channels and quantum hypothesis testing. IEEE Transactions on Information Theory, 64(2):1385-1403, Feb 2018.

[45] H. C. Cheng, M. H. Hsieh, and M. Tomamichel. Quantum sphere-packing bounds with polynomial prefactors. IEEE Transactions on Information Theory, 65(5):2872-2898, May 2019. (arXiv:1704.05703 [quant-ph]).

[46] Y. Altuğ and A. B. Wagner. Refinement of the random coding bound. IEEE Transactions on Information Theory, 60(10):6005-6023, Oct 2014.

[47] J. Scarlett, A. Martinez, and A. G. i Fabregas. Mismatched decoding: Error exponents, second-order rates and saddlepoint approximations. IEEE Transactions on Information Theory, 60(5):2647-2666, May 2014.

[48] J. Honda. Exact asymptotics for the random coding error probability. In 2015 IEEE International Symposium on Information Theory (ISIT), pages 91-95, June 2015 
[49] M. V. Burnashev and A. S. Holevo. On the reliability function for a quantum communication channel. Problems of Information Transmission, 34(2):7107, 1998.

[50] E. Telatar. Capacity of multi-antenna gaussian channels. European Transactions on Telecommunications, 10(6):585-595, 1999.

[51] M. Vu. MISO capacity with per-antenna power constraint. IEEE Transactions on Communications, 59(5):1268-1274, May 2011.

[52] P. L. Cao, T. J. Oechtering, R. F. Schaefer, and M. Skoglund. Optimal transmit strategy for MISO channels with joint sum and per-antenna power constraints. IEEE Transactions on Signal Processing, 64(16):4296-4306, Aug 2016.

[53] S. Loyka. The capacity of gaussian MIMO channels under total and per-antenna power constraints. IEEE Transactions on Communications, 65(3):10351043, March 2017.

54] H.-C. Cheng and B. Nakiboğlu. Refined strong converse for the constant composition codes. arXiv:2002.11414, 2020.

[55] Wei Yang. Fading Channels: Capacity and Channel Coding Rate in the Finite-Blocklength Regime. Communication systems group, Department of Signals and Systems Chalmers at University of Technology, Gothenburg, Sweden, 2015. 